\title{
Three-dimensional Wentzel-Kramers-Brillouin approach for the simulation of scanning tunneling microscopy and spectroscopy
}

\author{
Krisztián Palotás ${ }^{1, *}$, Gábor Mándi ${ }^{2}$, Werner A. Hofer ${ }^{3, \dagger}$ \\ ${ }^{1}$ Department of Theoretical Physics, Budapest University of Technology and Economics, and Condensed Matter Research \\ Group of the Hungarian Academy of Sciences, Budafoki út 8., H-1111 Budapest, Hungary \\ ${ }^{2}$ Department of Theoretical Physics, Budapest University of Technology and Economics, Budafoki út 8., H-1111 Budapest, Hungary \\ ${ }^{3}$ Surface Science Research Centre, University of Liverpool, L69 3BX Liverpool, UK \\ E-mail: *palotas@phy.bme.hu, ${ }^{\dagger}$ whofer@liverpool.ac.uk \\ Received April 18, 2013; accepted May 25, 2013
}

We review the recently developed three-dimensional (3D) atom-superposition approach for simulating scanning tunneling microscopy (STM) and spectroscopy (STS) based on $a b$ initio electronic structure data. In the method, contributions from individual electron tunneling transitions between the tip apex atom and each of the sample surface atoms are summed up assuming the one-dimensional (1D) Wentzel-Kramers-Brillouin (WKB) approximation in all these transitions. This 3D WKB tunneling model is extremely suitable to simulate spin-polarized STM and STS on surfaces exhibiting a complex noncollinear magnetic structure, i.e., without a global spin quantization axis, at very low computational cost. The tip electronic structure from first principles can also be incorporated into the model, that is often assumed to be constant in energy in the vast majority of the related literature, which could lead to a misinterpretation of experimental findings. Using this approach, we highlight some of the electron tunneling features on a prototype frustrated hexagonal antiferromagnetic $\mathrm{Cr}$ monolayer on $\mathrm{Ag}(111)$ surface. We obtain useful theoretical insights into the simulated quantities that is expected to help the correct evaluation of experimental results. By extending the method to incorporate a simple orbital dependent electron tunneling transmission, we reinvestigate the bias voltage- and tip-dependent contrast inversion effect on the W(110) surface. STM images calculated using this orbital dependent model agree reasonably well with Tersoff-Hamann and Bardeen results. The computational efficiency of the model is remarkable as the $k$-point samplings of the surface and tip Brillouin zones do not affect the computational time, in contrast to the Bardeen method. In a certain case we obtain a relative computational time gain of 8500 compared to the Bardeen calculation, without the loss of quality. We discuss the advantages and limitations of the $3 \mathrm{D}$ WKB method, and show further ways to improve and extend it.

Keywords electron tunneling, STM, STS, WKB, tunneling transmission, spin polarization, metal surfaces, contrast inversion

PACS numbers 68.37.Ef, 71.15.-m, 72.25.Ba, 73.22.-f, 73.63.-b

Contents

1 Introduction

2 Theory

2.1 Theoretical model of SP-STM within the 3D WKB approach
2.1.1 Density matrix formalism

2.1.2 Transmission

2.1.3 Sample and tip electronic properties

2.1.4 Tunneling current

2.2 Theoretical model of SP-STS within the 3D WKB approach

(C) Higher Education Press and Springer-Verlag Berlin Heidelberg 2013 
2.3 Orbital dependent tunneling within the 3D WKB approach

2.4 Tunneling properties

2.4.1 Current

2.4.2 Contrast

2.4.3 Differential conductance

2.4.4 Magnetic asymmetry

3 Prototype noncollinear magnetic surface: $\mathrm{Cr} / \mathrm{Ag}(111)$

3.1 Computational details

3.2 Magnetic ground state

3.3 SP-STM detection of magnetic chirality

3.4 Tip effects on the SP-STM images

3.4.1 Tip material effect on the magnetic contrast

3.4.2 Tip-sample distance effect on the magnetic contrast

3.4.3 Tip magnetization direction effect on the magnetic contrast

$3.5 \mathrm{~d} I / \mathrm{d} V$ contributions

3.6 Tip effects on the SP-STS spectra

3.7 Tip effects on the magnetic asymmetry

3.8 Two-dimensional local spectroscopic information

4 Corrugation inversion due to orbital effects:

$\mathrm{W}(110)$

4.1 Computational details

4.2 Convergence properties

4.3 Orbital contributions

4.4 Atomic contrast reversal

4.5 STM images - Comparison to other tunneling models

5 Summary and outlook

Acknowledgements

References

\section{Introduction}

The scanning tunneling microscopy (STM) and spectroscopy (STS) techniques become routine and extremely useful high resolution surface science tools for the investigation of local physical and chemical phenomena on surfaces of materials since the invention of the STM 31 years ago $[1,2]$. The experimental success in the continuous development of these techniques and the unraveling of the physics of a mass of new technologically relevant phenomena calls for the development of theoretical and simulation tools that are inevitable for the correct interpretation of STM and STS measurements $[3,4]$. Beside highly accurate but computationally very demanding models that are routinely available to theorist experts only, there is a need for simulation tools that could be easy to use for experimental groups, and 9 give results within minutes with reasonable accuracy. For 11 this reason, we propose a three-dimensional (3D) atom11 superposition approach for simulating STM and STS. 12 We review the formalism of this method and the ob13 tained results so far, that give important insights into 13 the structure of the measured tunneling current, differential conductance and related quantities. Moreover, the 14 results provide useful instructions for the correct inter15 pretation of spin-polarized STM (SP-STM) and spin15 polarized STS (SP-STS) measurements, and facilitate 16 a new route to understand the bias voltage- and tip16 dependent atomic contrast inversion phenomenon observed on metallic surfaces by highlighting the role of the real space shape of the orbitals involved in the tunneling. The key input for the tunneling model is the projected electron density of states (PDOS) of the tip apex atom and of the sample surface atoms obtained from $a b i n i$ tio electronic structure calculations. These can be calculated using any suitable computational code. The basic idea of the model is that contributions from individual electron tunneling transitions between the tip apex atom and each of the sample surface atoms are summed up Brillouin (WKB) approximation in all these transitions. Therefore, this tunneling model is a 3D WKB approach.

First, we briefly review the experimental and theoretical motivations that led to the construction of this $3 \mathrm{D}$ WKB model.

One direction of recent theoretical research is focused on extracting surface local electronic properties from experimental STS data [5-9], which is the convolution of tip and sample electronic structures. Applying STS in the spin-polarized setup, this research line could be extended to the possible extraction of local spin polarization information of surfaces. Here, special attention has to be taken to the composition of the differential conductance $(\mathrm{d} I / \mathrm{d} V)$, and it has been suggested that the magnetic asymmetry defined from experimentally measured spectra does not correspond to the effective spin polarization [10].

Another research direction is concerned with the simulation of STM and STS by using different models mostly based on electronic structure data obtained from first principles. A computationally demanding but very accurate method is based on nonequilibrium Green functions [11] that has been mostly applied for ballistic electron transport. More traditional approaches are based on the work of Tersoff and Hamann [12, 13], and Bardeen [14]. Wortmann et al. [15] introduced the spin-polarized version of the Tersoff-Hamann model.

Much work has been devoted to analyze the electron tunneling properties depending on the scanning tip. In 
Ref. [16] a theoretical method has been presented to separate the tip and sample contributions to STS. A related study has been performed experimentally [17]. Ness and Gautier studied different metal tips and their interaction with metal surfaces in a tight-binding framework [18-20]. Chen and Sacks investigated the effect of the tip orbitals on the corrugation of constant current STM images theoretically $[21,22]$. While Chen pointed out that corrugation enhancement is expected for tip orbitals localized along the surface normal $(z)$ direction $\left(p_{z}\right.$ and $\left.d_{3 z^{2}-r^{2}}\right)$, Sacks argued that $m \neq 0$ tip states $\left(d_{x z}, d_{y z}\right.$, $\left.d_{x y}, d_{x^{2}-y^{2}}\right)$ are responsible for this effect. Another work of Chen explained the corrugation inversion found on low Miller index metal surfaces by $m \neq 0$ tip states [23]. Atomic contrast reversal has also been found above Xe atomic adsorbates [24] and oxygen overlayers [25] on metal surfaces. It was established that the character of the contrast depends on the tip-sample distance and on the tip geometry and electronic structure. The quality of the tip also plays a crucial role in the inelastic tunneling spectroscopy (IETS). Studying the CO molecule on $\mathrm{Cu}$ surfaces it has been found that the IETS intensity is close to the experiment including the full electronic structure of the tip [26], and the tip position and orbital symmetry can change the selection rules for the active vibrational modes in IETS [27]. Moreover, it was proposed that IETS could be a useful tool to get information about the tip apex structure [28].

The role of the electron orbitals has been considered in different electron transport models. Sirvent et al. developed a tight-binding model based on the Keldysh formalism for calculating the conductance in atomic point contacts and analyzed the effect of the $d$ orbitals [29]. The same methodology has been applied to STM junctions by Mingo et al. [24]. Cerdá et al. presented an STM simulation method based on the Landauer-Büttiker formula [30] and the surface Green function matching technique [31]. In these methods the decomposition of the conductance and/or current with respect to electron orbitals has been given. The conductance can also be decomposed in terms of transmission eigenchannels. There are many examples for atomic point contacts, see, e.g., Refs. [32-35], and such decomposition is also possible in STM junctions [36].

An emerging research field in surface science is the investigation of magnetism at the nanoscale and atomic scale with the aim of achieving ultrahigh information density for data storage purposes [37, 38]. This can be achieved by reducing the size of the information storage units going down to the nanoscale or even to single atoms [39]. Detecting and manipulating spins [40] with high accuracy on the atomic scale is essential for future technological applications. Recent experimental advances using the SP-STM technique allow the investigation of complex magnetic structures (frustrated antiferromagnets, spin spirals, skyrmion lattices, etc.) [39, 41-48]. Considering such structures in reduced dimensions, their magnetic ground state can be determined [46, 49-52], and the nature of magnetic interactions can be studied by theoretical means, e.g., from first principles [53], or applying a multiscale approach [54]. Using the theoretically calculated electronic structure in the magnetic ground state, electron tunneling transport properties can be investigated.

In the most routinely used constant current mode of the SP-STM the apparent height difference between differently magnetized surface atoms allows the discrimination of the individual atomic magnetic properties and the mapping of the magnetic structure. This apparent height difference is called the magnetic contrast. Finding the maximal magnetic contrast for a given surfacetip combination is crucial for a more efficient magnetic mapping. This can be done by using magnetic tips with large spin polarization [42], and/or by choosing an appropriate bias voltage that selects highly spin-polarized tunneling states. A very few works focused on the investigation of the bias dependent magnetic contrast so far. Among those, a magnetic contrast reversal was reported in two different magnetic systems [55, 56], and the effect was related to the surface electronic structure rather than to the effect of the tip. In another study, such contrast reversals were observed during the scanning with the STM tip at fixed bias, and this was identified to be due to the magnetic switching of the tip [46]. An extensive work on the prediction of the bias dependent magnetic contrast based on a set of single point measurements is presented in Ref. [57]. Moreover, under certain circumstances, a giant magnetic contrast can be obtained, and this effect was explained by chemically modified STM tips [58]. Small contrast variations owing to the tunneling anisotropic magnetoresistance (TAMR) effect have also been reported $[59,60]$. This effect is due to the spin-orbit interaction that modifies the local electronic structure of the surface, and it can be used as a nonmagnetic probe for magnetic properties.

The spectroscopic operating mode of STM, the STS can be applied to investigate many-body effects on substrate-supported adatoms [61-68], and molecules [6971], where Fano resonances [72] contain characteristic information about the Kondo physics. SP-STS has recently been used to find inversion of spin polarization above magnetic adatoms [73-75], and the effect has been explained theoretically [76]. Furthermore, SP-STS is useful to study atomic magnetism [77], magnetic interactions between adatoms [78], and magnetic nanostructures on surfaces, see, e.g., Refs. [79-83]. This technique has re- 
cently been used to read out information from an atomic scale all-spin-based logic device [84]. The effect of differently magnetized surface regions on SP-STS has also been reported $[79,85]$, and the role of tip effects on STS $[7,86,87]$ and SP-STS $[10,88,89]$ have been highlighted.

Let us now continue with the description of the $3 \mathrm{D}$ WKB model.

\section{Theory}

Based on the work of Heinze [90], a hybrid model has been proposed [56], that uses essentially the TersoffHamann formalism but the tip electron density of states (DOS) is not restricted to be constant in energy. This means that different tip models [76] and their effect on the tunneling properties can be investigated. The only requirement for the presented formalism is that it is assumed that electrons tunnel through one tip apex atom. Since the tip electronic structure is explicitly included in this method via the projected DOS onto the apex atom, Eq. (2) of Ref. [90] needs to be reconsidered. The strategy is to determine differential currents first, and then perform an energy integral in a window according to the applied bias voltage $(V)$ in order to arrive at the tunneling current. The differential conductance can then be obtained by the bias-derivative of the current, containing three characteristic terms [10].

First, we review the tunneling current and the differential conductance expressions based on the independent orbital approximation for the vacuum decay of electron states, and then we extend this model to include a simple orbital dependent tunneling transmission [91]. Evaluation of the simulated tunneling properties from a data set on a 3D fine grid above the studied surface is presented thereafter. This includes the extraction of SP-STM images [56], magnetic contrasts [57], differential conductance and effective spin polarization maps [10], as well as magnetic asymmetries [10].

2.1 Theoretical model of SP-STM within the 3D WKB approach

In SP-STM the total tunneling current can be decomposed into a non-spin-polarized (topographic), $I_{\mathrm{TOPO}}$, and a spin-polarized (magnetic) part, $I_{\mathrm{MAGN}}[15,90,92$, 93],

$$
I_{\mathrm{TOTAL}}=I_{\mathrm{TOPO}}+I_{\mathrm{MAGN}}
$$

This formula is generally valid for either collinear or noncollinear surface and tip spin structures. $I_{\mathrm{TOPO}}$ and $I_{\text {MAGN }}$ can be calculated at different levels of transport theories. While the perturbation approach has been used by Hofer and Fisher [92] for collinear surface and tip spin structures with an arbitrary angle between their spin quantization axes, the most commonly used method is based on the Tersoff-Hamann model $[12,13]$. Wortmann et al. [15] introduced its spin-polarized version applicable to complex noncollinear surface spin structures. Heinze [90] combined it with the atom superposition method $[93,94]$. Note that the SP-STM method of Heinze does not consider bias voltage dependence of the tunneling current and related quantities.

\subsubsection{Density matrix formalism}

Let us define the following position- and energydependent density matrices in spin space for the tip (T) and sample (S), respectively,

$$
\underline{\underline{\rho}}_{\mathrm{T}, \mathrm{S}}(\boldsymbol{r}, E)=n_{\mathrm{T}, \mathrm{S}}(\boldsymbol{r}, E) \underline{\underline{I}}+\boldsymbol{m}_{\mathrm{T}, \mathrm{S}}(\boldsymbol{r}, E) \underline{\underline{\boldsymbol{\sigma}}}=\left[\begin{array}{cc}
n_{\mathrm{T}, \mathrm{S}}(\boldsymbol{r}, E)+m_{\mathrm{T}, \mathrm{S}}^{z}(\boldsymbol{r}, E) & m_{\mathrm{T}, \mathrm{S}}^{x}(\boldsymbol{r}, E)-\mathrm{i} m_{\mathrm{T}, \mathrm{S}}^{y}(\boldsymbol{r}, E) \\
m_{\mathrm{T}, \mathrm{S}}^{x}(\boldsymbol{r}, E)+\mathrm{i} m_{\mathrm{T}, \mathrm{S}}^{y}(\boldsymbol{r}, E) & n_{\mathrm{T}, \mathrm{S}}(\boldsymbol{r}, E)-m_{\mathrm{T}, \mathrm{S}}^{z}(\boldsymbol{r}, E)
\end{array}\right]
$$

Here, $\underline{\underline{I}}$ is the $2 \times 2$ unit matrix and $\underline{\underline{\boldsymbol{\sigma}}}$ is the Pauli matrix vector. The charge and magnetization DOS at position $\boldsymbol{r}$ and energy $E, n_{\mathrm{T}, \mathrm{S}}(\boldsymbol{r}, E)$ and $\boldsymbol{m}_{\mathrm{T}, \mathrm{S}}(\boldsymbol{r}, E)$, respectively, can be obtained from the corresponding density matrix as

$$
\begin{aligned}
& n_{\mathrm{T}, \mathrm{S}}(\boldsymbol{r}, E)=\frac{1}{2} \operatorname{Tr}\left(\underline{\rho}_{\mathrm{T}, \mathrm{S}}(\boldsymbol{r}, E) \underline{\underline{I}}\right) \\
& \left.\boldsymbol{m}_{\mathrm{T}, \mathrm{S}}(\boldsymbol{r}, E)=\frac{1}{2} \operatorname{Tr} \underline{\underline{\rho}}_{\mathrm{T}, \mathrm{S}}(\boldsymbol{r}, E) \underline{\underline{\boldsymbol{\sigma}}}\right)
\end{aligned}
$$

where the trace is performed in the spin space. In the absence of the tip material, the charge and magnetization electron local density of states (LDOS) of the sample surface at the tip position $\boldsymbol{R}_{\mathrm{TIP}}$ in the vacuum, respectively $n_{\mathrm{S}}\left(\boldsymbol{R}_{\mathrm{TIP}}, E\right)$ and $\boldsymbol{m}_{\mathrm{S}}\left(\boldsymbol{R}_{\mathrm{TIP}}, E\right)$, are obtained.
$n_{\mathrm{T}}\left(\boldsymbol{R}_{\mathrm{TIP}}, E\right)$ and $\boldsymbol{m}_{\mathrm{T}}\left(\boldsymbol{R}_{\mathrm{TIP}}, E\right)$ are the charge and magnetization DOS projected to the tip apex atom. Combining the sample and tip density matrices at $\boldsymbol{R}_{\mathrm{TIP}}$, a modified LDOS, denoted by MLDOS, can be defined as

$$
\begin{aligned}
& M L D O S\left(\boldsymbol{R}_{\mathrm{TIP}}, E\right) \\
&=\varepsilon \frac{1}{2} \operatorname{Tr}\left(\underline{\rho}_{\mathrm{S}}\left(\boldsymbol{R}_{\mathrm{TIP}}, E\right) \underline{\rho}_{\mathrm{T}}\left(\boldsymbol{R}_{\mathrm{TIP}}, E\right)\right) \\
&=\varepsilon\left[n_{\mathrm{S}}\left(\boldsymbol{R}_{\mathrm{TIP}}, E\right) n_{\mathrm{T}}\left(\boldsymbol{R}_{\mathrm{TIP}}, E\right)\right. \\
&\left.+\boldsymbol{m}_{\mathrm{S}}\left(\boldsymbol{R}_{\mathrm{TIP}}, E\right) \boldsymbol{m}_{\mathrm{T}}\left(\boldsymbol{R}_{\mathrm{TIP}}, E\right)\right]
\end{aligned}
$$

that, in fact, combines the vacuum LDOS of the surface and the projected DOS of the tip apex atom. This formula is consistent with the spin-polarized TersoffHamann model [15], except the fact that it explicitly 
includes the energy-dependent electronic structure of the tip apex. Here, $\varepsilon$ ensures that the MLDOS is correctly measured in the units of $(\mathrm{eV})^{-1}$. In the following, the notations $n_{\mathrm{T}}(E)=n_{\mathrm{T}}\left(\boldsymbol{R}_{\mathrm{TIP}}, E\right)$ and $\boldsymbol{m}_{\mathrm{T}}(E)=$ $\boldsymbol{m}_{\mathrm{T}}\left(\boldsymbol{R}_{\mathrm{TIP}}, E\right)$ are used.

The position dependence of the sample density matrices are considered as $\underline{\rho}_{\mathrm{S}}\left(\boldsymbol{R}_{a}, E\right)$ with $\boldsymbol{R}_{a}$ the position vector of the ath sample surface atom, in order to allow different chemical or magnetic properties for these atoms. $n_{\mathrm{S}}^{a}(E)=n_{\mathrm{S}}\left(\boldsymbol{R}_{a}, E\right)$ and $\boldsymbol{m}_{\mathrm{S}}^{a}(E)=\boldsymbol{m}_{\mathrm{S}}\left(\boldsymbol{R}_{a}, E\right)$ now denote charge and magnetization DOS projected to the $a$ th surface atom, respectively. Following this, the vacuum LDOS of the surface can be approximated by a superposition of exponentially decaying atomic electron states. This way, the electron charge and magnetization LDOS at energy $E$ and at the $\boldsymbol{R}_{\mathrm{TIP}}(x, y, z)$ position in the vacuum above the surface, $n_{\mathrm{S}}(x, y, z, E)$ and $\boldsymbol{m}_{\mathrm{S}}(x, y, z, E)$, respectively, are given by the following expressions:

$$
\begin{aligned}
& n_{\mathrm{S}}(x, y, z, E)=\sum_{a} \mathrm{e}^{-2 \kappa(E) d_{a}(x, y, z)} n_{\mathrm{S}}^{a}(E) \\
& \boldsymbol{m}_{\mathrm{S}}(x, y, z, E)=\sum_{a} \mathrm{e}^{-2 \kappa(E) d_{a}(x, y, z)} \boldsymbol{m}_{\mathrm{S}}^{a}(E)
\end{aligned}
$$

with

$$
\kappa(E)=\frac{1}{\hbar} \sqrt{2 m\left(\phi_{\mathrm{S}}+E_{\mathrm{F}}^{\mathrm{S}}-E\right)}
$$

where the electron's mass is $m$, $\hbar$ is the reduced Planck constant, and $\phi_{\mathrm{S}}$ and $E_{\mathrm{F}}^{\mathrm{S}}$ are the average electron work function and the Fermi energy of the sample surface, respectively. Here,

$$
d_{a}(x, y, z)=\left|\boldsymbol{R}_{\mathrm{TIP}}(x, y, z)-\boldsymbol{R}_{a}\right|
$$

is the distance between the tip apex and the ath surface atom. Note that the exact vacuum LDOS of the surface can be obtained, in principle, by explicitly calculating the decay of the electron states into the vacuum taking their orbital symmetry into account as well, not just via such a simple atom-decomposed model. This atom superposition approach, however, has computational advantages as will be demonstrated later.

An elastic tunneling transition between the tip apex and the $a$ th surface atom at energy $E$ can be represented as the trace of the multiplied density matrices, similarly to Eq. (5). This is the energetic ingredient for the tunneling transition. Apart from this, the transmission coefficient through a potential barrier between the ath surface atom and the tip apex has to be included in the tunneling model, in general it is denoted by $T\left(E, V, d_{a}\right)$. It has energy $(E)$ and bias $(V)$ dependence, and contains a tipposition-dependent geometry information of the threedimensional tunnel junction via the set of $d_{a}\left(\boldsymbol{R}_{\mathrm{TIP}}\right)$.

Thus, the modified LDOS at the tip apex position
$\boldsymbol{R}_{\mathrm{TIP}}(x, y, z)$ and at energy $E$ can be approximated as the superposition of individual atomic contributions from the sample surface as

$$
\begin{array}{rl}
M & L D O S\left(\boldsymbol{R}_{\mathrm{TIP}}, E, V\right)=\varepsilon \sum_{a} T\left(E, V, d_{a}\left(\boldsymbol{R}_{\mathrm{TIP}}\right)\right) \\
& \times \frac{1}{2} \operatorname{Tr}\left(\underline{\underline{\rho}}_{\mathrm{S}}\left(\boldsymbol{R}_{a}, E\right) \underline{\underline{\rho}}_{\mathrm{T}}\left(\boldsymbol{R}_{\mathrm{TIP}}, E\right)\right)
\end{array}
$$

The main advantage of using the density matrix formalism is that electronic and spin structures calculated either nonrelativistically or relativistically can be treated within the same theoretical framework. Moreover, the motivation for using the atomic superposition approximation is, on one hand, computational efficiency, since calculating and storing the projected DOS onto surface atoms in the magnetic unit cell is computationally much cheaper compared to the exact vacuum LDOS of the surface on a great number of grid points. On the other hand, such atom-projected PDOS functions are routinely obtained in all $a b$ initio electronic structure codes, whereas the vacuum LDOS is not always routinely accessible for the average user.

\subsubsection{Transmission}

According to above, the MLDOS can be decomposed, similarly to Eq. (1), as

$$
\begin{aligned}
& M L D O S(x, y, z, E, V) \\
& =M L D O S_{\mathrm{TOPO}}(x, y, z, E, V) \\
& +\operatorname{MLDOS}_{\mathrm{MAGN}}(x, y, z, E, V)
\end{aligned}
$$

and assuming an exponentially decaying tunneling transmission,

$$
T\left(E, V, d_{a}(x, y, z)\right)=\mathrm{e}^{-2 \kappa(E, V) d_{a}(x, y, z)}
$$

the TOPO and MAGN terms can be written as

$$
\begin{aligned}
& M^{M} L_{D O S_{\mathrm{TOPO}}(x, y, z, E, V)} \\
& \quad=\varepsilon \sum_{a} \mathrm{e}^{-2 \kappa(E, V) d_{a}(x, y, z)} n_{\mathrm{T}}(E) n_{\mathrm{S}}^{a}(E) \\
& M L D O S_{\mathrm{MAGN}}(x, y, z, E, V) \\
& \quad=\varepsilon \sum_{a} \mathrm{e}^{-2 \kappa(E, V) d_{a}(x, y, z)} \boldsymbol{m}_{\mathrm{T}}(E) \boldsymbol{m}_{\mathrm{S}}^{a}(E)
\end{aligned}
$$

Here, the sum over $a$ has to be carried out, in principle, over all the surface atoms. Convergence tests, however, showed that including a relatively small number of atoms in the sum provides converged MLDOS values [89]. The exponential factor in these equations is the transmission probability for electrons tunneling between states of atom $a$ on the surface and the tip apex, where $\kappa$ is the vacuum decay. $\kappa$ is treated within the independentorbital approximation $[12,13,90]$, that means that the 
same spherical decay is assumed for all electron states irrespective of their orbital symmetry, but its energy dependence is explicitly considered essentially in the same fashion as in Ref. [95]. Extension of this model to take into account orbital dependent tunneling transmission is reported in Section 2.3. Two different ways of calculating $\kappa$ are considered. One is inspired by the Tersoff-Hamann model, taking only surface properties into account, see Eq. (8). Note that this formula does not have an explicit bias dependence. This energy-dependent vacuum decay can be used for an ideal, electronically featureless and maximally spin-polarized tip model. Taking into account the tip apex electronic structure obtained from first principles, the more general expression for $\kappa$ is based on the 1D WKB approximation assuming an effective rectangular potential barrier between the tip and the surface,

$$
\kappa(E, V)=\frac{1}{\hbar} \sqrt{2 m\left(\frac{\phi_{\mathrm{S}}+\phi_{\mathrm{T}}+e V}{2}+E_{\mathrm{F}}^{\mathrm{S}}-E\right)}
$$

with $\phi_{\mathrm{T}}$ the local electron work function of the tip apex, $e$ the elementary charge, and $V$ the applied bias voltage. This vacuum decay formula is considered for different model tip materials. The quantity $\left(\phi_{\mathrm{S}}+\phi_{\mathrm{T}}+e V\right) / 2+$ $E_{\mathrm{F}}^{\mathrm{S}}-E$ is the energy- and bias-dependent apparent barrier height for tunneling electrons, $\phi_{a p p}(E, V)$. Empirical or model nonlinear variations of $\phi_{a p p}(E, V)$ with respect to the bias voltage $[96,97]$ can also be included in this approach. Note that in the case of $\phi_{\mathrm{T}}+e V=\phi_{\mathrm{S}}$, Eq. (8) is recovered. The average work function of the sample surface is calculated from the local electrostatic potential on a three-dimensional fine grid, $\Phi(x, y, z)$, as

$$
\phi_{\mathrm{S}}=\max _{z}\left\{\frac{1}{N_{x} N_{y}} \sum_{x, y} \Phi(x, y, z)\right\}-E_{\mathrm{F}}^{\mathrm{S}}
$$

with $N_{x}$ and $N_{y}$ the corresponding number of grid points, and the local work function of the tip apex is obtained as

$$
\phi_{\mathrm{T}}=\max _{z}\left\{\Phi\left(x_{0}, y_{0}, z\right)\right\}-E_{\mathrm{F}}^{\mathrm{T}}
$$

with $x_{0}$ and $y_{0}$ lateral coordinates of the tip apex atom, and $E_{F}^{T}$ the Fermi energy of the tip.

\subsubsection{Sample and tip electronic properties}

The material properties are included into this tunneling model via the projected electron density of states (PDOS) of the tip apex and of the sample surface atoms that can be obtained from collinear or noncollinear electronic structure calculations. In Eq. (14), $\boldsymbol{m}_{\mathrm{T}}(E)$ and $\boldsymbol{m}_{\mathrm{S}}^{a}(E)$ denote electron magnetization DOS vectors projected to the tip apex and the ath surface atom, respectively. In the collinear case,

$$
\begin{aligned}
& \boldsymbol{m}_{\mathrm{T}, \mathrm{S}}(E)=m_{\mathrm{T}, \mathrm{S}}(E) \boldsymbol{e}_{\mathrm{T}, \mathrm{S}} \\
& m_{\mathrm{T}, \mathrm{S}}(E)=n_{\mathrm{T}, \mathrm{S}}^{\uparrow}(E)-n_{\mathrm{T}, \mathrm{S}}^{\downarrow}(E)
\end{aligned}
$$

$\uparrow$ and $\downarrow$ relative to their energy-independent local spin quantization axes, denoted by the unit vectors $\boldsymbol{e}_{\mathrm{T}, \mathrm{S}}$. Similarly, in Eq. (13), $n_{\mathrm{T}}(E)$ and $n_{\mathrm{S}}^{a}(E)$ are the electron charge DOS projected to the tip apex and the ath surface atom, respectively. They can be written in the collinear case as

$$
n_{\mathrm{T}, \mathrm{S}}(E)=n_{\mathrm{T}, \mathrm{S}}^{\uparrow}(E)+n_{\mathrm{T}, \mathrm{S}}^{\downarrow}(E)
$$

The spin-resolved PDOS quantities, $n_{\mathrm{T}, \mathrm{S}}^{\uparrow, \downarrow}(E)$, can be obtained from first principles collinear magnetic calculations. For this task any available $a b$ initio electronic structure code can be used. Spin-resolved PDOS is calculated by assuming a Gaussian broadening of the peaks at the $k$-resolved spin-dependent electron energy (KohnSham) eigenvalues, $\varepsilon_{\mathrm{T}, \mathrm{S}}^{j \uparrow, \boldsymbol{k}}$, obtained at zero temperature, as

$$
\begin{aligned}
n_{\mathrm{T}, \mathrm{S}}^{\uparrow, \downarrow}(E)= & \sum_{\boldsymbol{k}} \sum_{j} \frac{1}{G \sqrt{\pi}} \mathrm{e}^{-\left(E-\varepsilon_{\mathrm{T}, \mathrm{S}}^{j \uparrow, \downarrow}(\boldsymbol{k})\right)^{2} / G^{2}} \\
& \times \int_{\text {atomic volume }} \mathrm{d}^{3} r \psi_{\mathrm{T}, \mathrm{S}}^{j \boldsymbol{k} \uparrow, \downarrow \dagger}(\boldsymbol{r}) \psi_{\mathrm{T}, \mathrm{S}}^{j \boldsymbol{k} \uparrow, \downarrow}(\boldsymbol{r})
\end{aligned}
$$

with $\psi_{T, S}^{j \boldsymbol{k} \uparrow, \downarrow}(\boldsymbol{r})$ the spin-dependent electron wave functions corresponding to $\varepsilon_{\mathrm{T}, \mathrm{S}}^{j \uparrow, \downarrow}(\boldsymbol{k})$ for the tip (T) and the surface (S), respectively, and $j$ the energy band index. The integral over the corresponding atomic volumes can be performed either in the atomic sphere or within the Bader volume [98]. The Gaussian parameter $G$ could, in general, be temperature dependent. In standard calculations, its value can be fixed to a relatively high value of $0.1 \mathrm{eV}$ in order to provide smooth $n_{\mathrm{T}, \mathrm{S}}^{\uparrow, \downarrow}(E)$ functions. Concerning the smoothness of the PDOS, a high $G$ value counteracts the effect of eventually underrepresented bulk states due to a slab geometry, and it is useful if the number of $k$-points in the Brillouin zone is restricted due to computational reasons.

Taking the fully noncollinear electronic structure, the atom-projected charge DOS at energy $E$ can be obtained as

$$
\begin{aligned}
n_{\mathrm{T}, \mathrm{S}}(E)= & \sum_{\boldsymbol{k}} \sum_{j} \frac{1}{G \sqrt{\pi}} \mathrm{e}^{-\left(E-\varepsilon_{\mathrm{T}, \mathrm{S}}^{j}(\boldsymbol{k})\right)^{2} / G^{2}} \\
& \times \int_{\text {atomic volume }} \mathrm{d}^{3} r \Psi_{\mathrm{T}, \mathrm{S}}^{j \boldsymbol{k} \dagger}(\boldsymbol{r}) \Psi_{\mathrm{T}, \mathrm{S}}^{j \boldsymbol{k}}(\boldsymbol{r})
\end{aligned}
$$

where $\varepsilon_{\mathrm{T}, \mathrm{S}}^{j}(\boldsymbol{k})$ is the set of electron energy (Kohn-Sham) eigenvalues at zero temperature, and $\Psi_{\mathrm{T}, \mathrm{S}}^{j \boldsymbol{k}}(\boldsymbol{r})$ the corresponding spinor electron wave functions. The atomprojected magnetization DOS vector at energy $E$ reads 


$$
\begin{aligned}
\boldsymbol{m}_{\mathrm{T}, \mathrm{S}}(E)= & \sum_{\boldsymbol{k}} \sum_{j} \frac{1}{G \sqrt{\pi}} \mathrm{e}^{-\left(E-\varepsilon_{\mathrm{T}, \mathrm{S}}^{j}(\boldsymbol{k})\right)^{2} / G^{2}} \\
& \times \int_{\text {atomic volume }} \mathrm{d}^{3} r \Psi_{\mathrm{T}, \mathrm{S}}^{j \boldsymbol{k} \dagger}(\boldsymbol{r}) \boldsymbol{\sigma} \Psi_{\mathrm{T}, \mathrm{S}}^{j \boldsymbol{k}}(\boldsymbol{r})
\end{aligned}
$$

with $\sigma$ being the Pauli spin operator vector. Unit vectors determining the local spin quantization axis of the tip apex, $\boldsymbol{e}_{\mathrm{T}}(E)$, and the ath surface atom, $\boldsymbol{e}_{\mathrm{S}}^{a}(E)$, at a given energy can be determined from these magnetization DOS vectors as

$$
\begin{aligned}
& \boldsymbol{e}_{\mathrm{T}, \mathrm{S}}(E)=\frac{\boldsymbol{m}_{\mathrm{T}, \mathrm{S}}(E)}{m_{\mathrm{T}, \mathrm{S}}(E)} \\
& =\frac{\boldsymbol{m}_{\mathrm{T}, \mathrm{S}}(E)}{\sqrt{m_{\mathrm{T}, \mathrm{S}}^{x}(E)^{2}+m_{\mathrm{T}, \mathrm{S}}^{y}(E)^{2}+m_{\mathrm{T}, \mathrm{S}}^{z}(E)^{2}}}
\end{aligned}
$$

thus, the atom-projected magnetization DOS vector has the following form:

$$
\begin{aligned}
& \boldsymbol{m}_{\mathrm{T}, \mathrm{S}}(E)=m_{\mathrm{T}, \mathrm{S}}(E) \boldsymbol{e}_{\mathrm{T}, \mathrm{S}}(E) \\
& =\sqrt{m_{\mathrm{T}, \mathrm{S}}^{x}(E)^{2}+m_{\mathrm{T}, \mathrm{S}}^{y}(E)^{2}+m_{\mathrm{T}, \mathrm{S}}^{z}(E)^{2}} \boldsymbol{e}_{\mathrm{T}, \mathrm{S}}(E)
\end{aligned}
$$

In general, the scalar product of the magnetization DOS of the tip apex and sample surface atoms in Eq. (14) can be rewritten as

$$
\begin{aligned}
\boldsymbol{m}_{\mathrm{T}}(E) \boldsymbol{m}_{\mathrm{S}}^{a}(E) & =m_{\mathrm{T}}(E) \boldsymbol{e}_{\mathrm{T}}(E) m_{\mathrm{S}}^{a}(E) \boldsymbol{e}_{\mathrm{S}}^{a}(E) \\
& =m_{\mathrm{T}}(E) m_{\mathrm{S}}^{a}(E) \cos \xi_{a}(E)
\end{aligned}
$$

Here, $\xi_{a}(E)$ is the angle between the spin quantization axes of the tip apex and the ath surface atom at energy $E$ defined by

$$
\cos \xi_{a}(E)=\boldsymbol{e}_{\mathrm{T}}(E) \boldsymbol{e}_{\mathrm{S}}^{a}(E)
$$

Previously, only the case of energy-independent $\xi_{a}$ has been considered [90], that corresponds to the fixed angle between the unit vectors of local spin quantization axes of surface atoms and the tip apex, $\boldsymbol{e}_{\mathrm{S}}^{a}$ and $\boldsymbol{e}_{\mathrm{T}}$, respectively. However, there are more possibilities to combine electronic structure data of the sample and the tip, that may result in an energy-dependent $\xi_{a}(E)$, see Table 1 . All listed combinations can be investigated within the presented formalism. The combination in the column with underlined values corresponds to the studied system in Ref. [89], and the combinations in columns in boldface are considered in Ref. [56].

The MLDOS can be rewritten in terms of energy dependent spin polarizations. The spin polarization is defined as

$$
P_{\mathrm{T}, \mathrm{S}}(E)=\frac{m_{\mathrm{T}, \mathrm{S}}(E)}{n_{\mathrm{T}, \mathrm{S}}(E)}=\frac{n_{\mathrm{T}, \mathrm{S}}^{\uparrow}(E)-n_{\mathrm{T}, \mathrm{S}}^{\downarrow}(E)}{n_{\mathrm{T}, \mathrm{S}}^{\uparrow}(E)+n_{\mathrm{T}, \mathrm{S}}^{\downarrow}(E)}
$$

assuming collinear electronic structure. From the noncollinear electronic structure the energy dependent spin polarization vectors are obtained by using Eq. (24) as

$$
\begin{aligned}
& \boldsymbol{P}_{\mathrm{T}, \mathrm{S}}(E)=\frac{\boldsymbol{m}_{\mathrm{T}, \mathrm{S}}(E)}{n_{\mathrm{T}, \mathrm{S}}(E)} \\
& =\frac{\sqrt{m_{\mathrm{T}, \mathrm{S}}^{x}(E)^{2}+m_{\mathrm{T}, \mathrm{S}}^{y}(E)^{2}+m_{\mathrm{T}, \mathrm{S}}^{z}(E)^{2}}}{n_{\mathrm{T}, \mathrm{S}}(E)} \boldsymbol{e}_{\mathrm{T}, \mathrm{S}}(E) \\
& =P_{\mathrm{T}, \mathrm{S}}(E) \boldsymbol{e}_{\mathrm{T}, \mathrm{S}}(E)
\end{aligned}
$$

Thus, the MLDOS at the tip apex position and at energy $E$ can alternatively be written using the above defined spin polarizations as

$$
\begin{aligned}
& M L D O S(x, y, z, E, V) \\
& =\varepsilon \sum_{a} \mathrm{e}^{-2 \kappa(E, V) d_{a}(x, y, z)} \\
& \quad \times n_{\mathrm{T}}(E) n_{\mathrm{S}}^{a}(E)\left[1+\boldsymbol{P}_{\mathrm{T}}(E) \boldsymbol{P}_{\mathrm{S}}^{a}(E)\right]
\end{aligned}
$$

where in the collinear case $\boldsymbol{e}_{\mathrm{T}, \mathrm{S}}$ has no energydependence:

$$
\boldsymbol{P}_{\mathrm{T}, \mathrm{S}}(E)=P_{\mathrm{T}, \mathrm{S}}(E) \boldsymbol{e}_{\mathrm{T}, \mathrm{S}}
$$

Table 1 Combinations of taking into account energy dependence $(\mathrm{Y})$ or independence $(\mathrm{N})$ of the local spin quantization axes of surface atoms $\left(\boldsymbol{e}_{\mathrm{S}}^{a}\right)$ and the tip apex $\left(\boldsymbol{e}_{\mathrm{T}}\right)$, and consequence for the energy dependence of the angle $\xi_{a}$ between the surface local ( $a$ th atom) and the tip quantization axes, where $\cos \xi_{a}=\boldsymbol{e}_{\mathrm{T}} \boldsymbol{e}_{\mathrm{S}}^{a}$. The combination in the column with underlined values corresponds to the studied system in Ref. [89], and the combinations in columns in boldface are considered in Ref. [56]. This Table is taken from Ref. [56].

\begin{tabular}{lccc}
\hline & $\begin{array}{c}\text { No. of atoms per } \\
\text { surface unit cell }\end{array}$ & $\begin{array}{c}\text { Surface } \\
\text { magnetic order }\end{array}$ & $\begin{array}{c}\text { Energy } \\
\text { dependence }\end{array}$ \\
\hline \multirow{2}{*}{$\boldsymbol{e}_{\mathrm{S}}^{a}(E)$} & 1 & N N Y Y \\
& $>1$ & Collinear & N N \\
& $>1$ & Noncollinear & N N Y Y \\
\hline $\boldsymbol{e}_{\mathrm{T}}(E)$ & & & N Y N Y N Y N Y N Y \\
\hline$\xi_{a}(E)$ & & $\underline{\text { N Y Y Y N Y N Y Y Y }}$ \\
\hline
\end{tabular}

\subsubsection{Tunneling current}

Using Eq. (11) of Ref. [15] and the MLDOS expressions in Eqs. (13) and (14), a virtual differential conductance at the tip apex position and at energy $E=E_{\mathrm{F}}^{\mathrm{S}}+e U$ can be defined for both the TOPO and MAGN contributions as

$$
\begin{aligned}
& \frac{\mathrm{d} I_{\mathrm{TOPO}}}{\mathrm{d} U}(x, y, z, U, V) \\
& =\varepsilon^{2} \frac{e^{2}}{h} \sum_{a} \mathrm{e}^{-2 \kappa\left(E_{\mathrm{F}}^{\mathrm{S}}+e U, V\right) d_{a}(x, y, z)} \\
& \quad \times n_{\mathrm{T}}\left(E_{\mathrm{F}}^{\mathrm{T}}+e U-e V\right) n_{\mathrm{S}}^{a}\left(E_{\mathrm{F}}^{\mathrm{S}}+e U\right)
\end{aligned}
$$




$$
\begin{aligned}
& \frac{\mathrm{d} I_{\text {MAGN }}}{\mathrm{d} U}(x, y, z, U, V) \\
& =\varepsilon^{2} \frac{e^{2}}{h} \sum_{a} \mathrm{e}^{-2 \kappa\left(E_{\mathrm{F}}^{\mathrm{S}}+e U, V\right) d_{a}(x, y, z)} \\
& \quad \times \boldsymbol{m}_{\mathrm{T}}\left(E_{\mathrm{F}}^{\mathrm{T}}+e U-e V\right) \boldsymbol{m}_{\mathrm{S}}^{a}\left(E_{\mathrm{F}}^{\mathrm{S}}+e U\right)
\end{aligned}
$$

where the fact is used that the tip Fermi level is shifted by $e V$ with respect to the sample Fermi level, i.e., $E_{\mathrm{F}}^{\mathrm{T}}=$ $E_{\mathrm{F}}^{\mathrm{S}}+e V$, and therefore $E=E_{\mathrm{F}}^{\mathrm{S}}+e U=E_{\mathrm{F}}^{\mathrm{T}}+e U-e V$, where $V$ is the bias voltage. Above formulas mean that multiplying the MLDOS by $\varepsilon$ results in a dimensionless quantity, that is multiplied by the conductance quantum $e^{2} / h$ in order to arrive at the $\mathrm{d} I / \mathrm{d} U$ expressions measured in the units of $A / V$. This means that both TOPO and MAGN parts of the $\mathrm{d} I / \mathrm{d} U$ is proportional to the corresponding MLDOS that contains both surface and tip electronic information. If the two subsystems are calculated separately, it is possible to combine different levels of electronic structure for the tip and the surface, see also Table 1, or include simplified model tip electronic structures into this approach.

The tunneling current can be determined from the $\mathrm{d} I / \mathrm{d} U$ quantities by integration in an energy window $\left[U_{1}(V, T), U_{2}(V, T)\right]$ corresponding to the applied bias voltage $(V)$ and temperature $(T)$ :

$$
\begin{aligned}
& I_{\mathrm{TOPO}}(x, y, z, V) \\
& \quad=\int_{U_{1}(V, T)}^{U_{2}(V, T)} \frac{\mathrm{d} I_{\mathrm{TOPO}}}{\mathrm{d} U}(x, y, z, U, V) \mathrm{d} U \\
& I_{\mathrm{MAGN}}(x, y, z, V) \\
& \quad=\int_{U_{1}(V, T)}^{U_{2}(V, T)} \frac{\mathrm{d} I_{\mathrm{MAGN}}}{\mathrm{d} U}(x, y, z, U, V) \mathrm{d} U
\end{aligned}
$$

where the energy window is defined as

$$
\begin{aligned}
& U_{1}(V, T)=\min (0, V)-\ln (3+\sqrt{8}) k_{\mathrm{B}} T / e \\
& U_{2}(V, T)=\max (0, V)+\ln (3+\sqrt{8}) k_{\mathrm{B}} T / e
\end{aligned}
$$

Here, $U=0$ corresponds to the Fermi energy of the sample surface, and $U=V$ to the Fermi energy of the tip. Broadening of electron states at finite temperatures is considered according to Eqs. (20)-(22), and the temperature dependent terms in the integral limits are the full width at half maximum of the energy-derivative of the Fermi distribution function divided by two, and $k_{\mathrm{B}}$ is the Boltzmann constant. Another, more precise way to include thermal effects in calculating the tunneling current is given in the Appendix of Ref. [86] based on the Sommerfeld expansion, which can also be incorporated into this approach. Lattice vibrations at nonzero temperatures are not taken into account. Assuming zero temperature, the corresponding tunneling currents can be obtained as

$$
I_{\mathrm{TOPO}}(x, y, z, V)=\int_{0}^{V} \frac{\mathrm{d} I_{\mathrm{TOPO}}}{\mathrm{d} U}(x, y, z, U, V) \mathrm{d} U
$$

$$
I_{\mathrm{MAGN}}(x, y, z, V)=\int_{0}^{V} \frac{\mathrm{d} I_{\mathrm{MAGN}}}{\mathrm{d} U}(x, y, z, U, V) \mathrm{d} U
$$

Note that fixing the integral limits as given here results in a sign change of the current components if $V<0$. One can calculate these current contributions at $(x, y, z)$ grid points of a $3 \mathrm{D}$ fine grid in a finite box above the surface, for a sketch see Fig. 1. The image resolution is determined by the density of $(x, y)$ grid points. More details about extracting constant height or constant current contours from the simulated data, i.e., generating SP-STM images, can be found in Section 2.4.1, and information on the image contrast is given in Section 2.4.2.

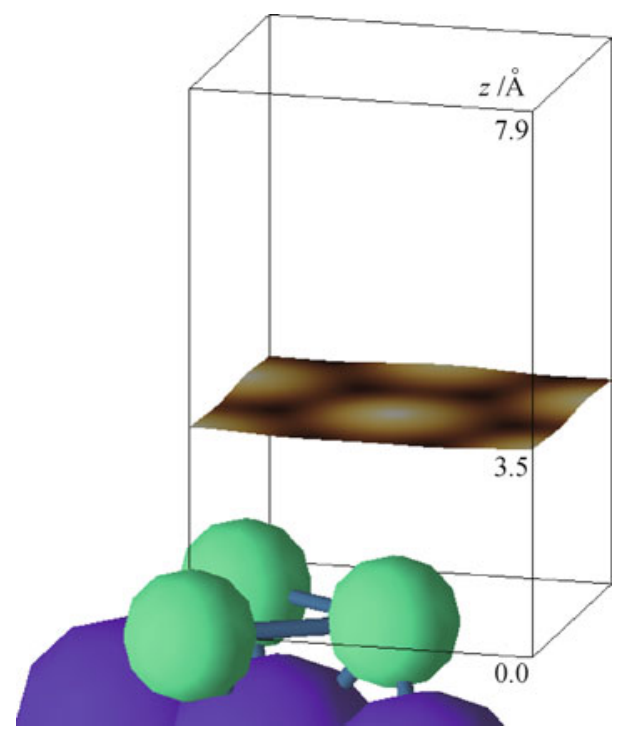

Fig. 1 Sketch of a finite box for data collection above a surface, and a constant current contour at about $3.5 \AA$.

2.2 Theoretical model of SP-STS within the 3D WKB approach

The differential conductance can be obtained as the derivative of the current with respect to the bias voltage. Assuming $T=0 \mathrm{~K}$ temperature,

$$
\begin{aligned}
& \frac{\mathrm{d} I}{\mathrm{~d} V}(x, y, z, V)=\frac{\mathrm{d} I}{\mathrm{~d} U}(x, y, z, V, V) \\
& \quad+\left.\int_{0}^{V} \frac{\partial}{\partial V^{\prime}} \frac{\mathrm{d} I}{\mathrm{~d} U}\left(x, y, z, U, V^{\prime}\right)\right|_{V^{\prime}=V} \mathrm{~d} U
\end{aligned}
$$

Similarly to the tunneling current, the differential conductance can be decomposed into non-spin-polarized (TOPO) and spin-polarized (MAGN) parts and it can be written at the tip position $\boldsymbol{R}_{\mathrm{TIP}}(x, y, z)$ and at bias voltage $V$ as

$$
\begin{aligned}
\frac{\mathrm{d} I_{\text {TOTAL }}}{\mathrm{d} V}(x, y, z, V)= & \frac{\mathrm{d} I_{\mathrm{TOPO}}}{\mathrm{d} V}(x, y, z, V) \\
& +\frac{\mathrm{d} I_{\mathrm{MAGN}}}{\mathrm{d} V}(x, y, z, V)
\end{aligned}
$$


In Eq. (39), the first term is the virtual differential conductance, the sum of Eqs. (31) and (32) at $U=V$. The second term can be reexpressed following the literature $[5,10,86]$, and the differential conductance at bias voltage $V$ can be written as the sum of three terms,

$$
\begin{aligned}
\frac{\mathrm{d} I}{\mathrm{~d} V}(x, y, z, V)= & \frac{\mathrm{d} I}{\mathrm{~d} U}(x, y, z, V, V)+B(x, y, z, V) \\
& +D_{\mathrm{T}}(x, y, z, V)
\end{aligned}
$$

Here, $B$ is the background term containing the biasderivative of the transmission function, and $D_{\mathrm{T}}$ is the tip-derivative term, containing the bias-derivative of the tip electronic structure. $B$ is usually taken into account in recent STS theories $\left[7,86,87\right.$, while $D_{\mathrm{T}}$ is rarely considered in the recent literature.

The contributions of Eq. (40) are given as

$$
\begin{aligned}
& \frac{\mathrm{d} I_{\mathrm{TOPO}}}{\mathrm{d} V}(x, y, z, V)=\frac{\mathrm{d} I_{\mathrm{TOPO}}}{\mathrm{d} U}(x, y, z, V, V) \\
& \quad+B_{\mathrm{TOPO}}(x, y, z, V)+D_{\mathrm{T}}^{\mathrm{TOPO}}(x, y, z, V) \\
& \frac{\mathrm{d} I_{\mathrm{MAGN}}}{\mathrm{d} V}(x, y, z, V)=\frac{\mathrm{d} I_{\mathrm{MAGN}}}{\mathrm{d} U}(x, y, z, V, V) \\
& \quad+B_{\text {MAGN }}(x, y, z, V)+D_{\mathrm{T}}^{\text {MAGN }}(x, y, z, V)
\end{aligned}
$$

and all terms in Eq. (41) can be written as the sum of TOPO and MAGN parts:

$$
\begin{aligned}
\frac{\mathrm{d} I}{\mathrm{~d} U}(x, y, z, V, V)= & \frac{\mathrm{d} I_{\mathrm{TOPO}}}{\mathrm{d} U}(x, y, z, V, V) \\
& +\frac{\mathrm{d} I_{\mathrm{MAGN}}}{\mathrm{d} U}(x, y, z, V, V) \\
B(x, y, z, V)= & B_{\mathrm{TOPO}}(x, y, z, V) \\
+ & B_{\mathrm{MAGN}}(x, y, z, V) \\
D_{\mathrm{T}}(x, y, z, V)= & D_{\mathrm{T}}^{\mathrm{TOPO}}(x, y, z, V) \\
& +D_{\mathrm{T}}^{\mathrm{MAGN}}(x, y, z, V)
\end{aligned}
$$

In order to calculate the background term, one needs the bias-derivative of the transmission function. Using Eq. (12) and the given form of the vacuum decay in Eq. (15), one obtains

$$
\begin{aligned}
& \frac{\partial T}{\partial V}\left(E_{\mathrm{F}}^{\mathrm{S}}+e U, V, d_{a}(x, y, z)\right) \\
& \quad=-\frac{m e}{\hbar^{2}} d_{a}(x, y, z) \frac{T\left(E_{\mathrm{F}}^{\mathrm{S}}+e U, V, d_{a}(x, y, z)\right)}{\kappa\left(E_{\mathrm{F}}^{\mathrm{S}}+e U, V\right)}
\end{aligned}
$$

Considering this, the background and the tip-derivative contributions can be written as

$$
\begin{aligned}
& B_{\mathrm{TO} O}(x, y, z, V)=-\varepsilon^{2} \frac{m e^{3}}{2 \pi \hbar^{3}} \sum_{a} d_{a}(x, y, z) \\
& \quad \times \int_{0}^{V} \frac{\mathrm{e}^{-2 \kappa\left(E_{\mathrm{F}}^{\mathrm{S}}+e U, V\right) d_{a}(x, y, z)}}{\kappa\left(E_{\mathrm{F}}^{\mathrm{S}}+e U, V\right)} \\
& \times n_{\mathrm{T}}\left(E_{\mathrm{F}}^{\mathrm{T}}+e U-e V\right) n_{\mathrm{S}}^{a}\left(E_{\mathrm{F}}^{\mathrm{S}}+e U\right) \mathrm{d} U
\end{aligned}
$$

$$
\begin{aligned}
& B_{\text {MAGN }}(x, y, z, V)=-\varepsilon^{2} \frac{m e^{3}}{2 \pi \hbar^{3}} \sum_{a} d_{a}(x, y, z) \\
& \times \int_{0}^{V} \frac{\mathrm{e}^{-2 \kappa\left(E_{\mathrm{F}}^{\mathrm{S}}+e U, V\right) d_{a}(x, y, z)}}{\kappa\left(E_{\mathrm{F}}^{\mathrm{S}}+e U, V\right)} \\
& \times \boldsymbol{m}_{\mathrm{T}}\left(E_{\mathrm{F}}^{\mathrm{T}}+e U-e V\right) \boldsymbol{m}_{\mathrm{S}}^{a}\left(E_{\mathrm{F}}^{\mathrm{S}}+e U\right) \mathrm{d} U \\
& D_{T}^{\mathrm{TOPO}}(x, y, z, V) \\
& =-\varepsilon^{2} \frac{e^{2}}{h} \sum_{a} \int_{0}^{V} \mathrm{e}^{-2 \kappa\left(E_{\mathrm{F}}^{\mathrm{S}}+e U, V\right) d_{a}(x, y, z)} \\
& \quad \times \frac{\partial n_{\mathrm{T}}}{\partial U}\left(E_{\mathrm{F}}^{\mathrm{T}}+e U-e V\right) n_{\mathrm{S}}^{a}\left(E_{\mathrm{F}}^{\mathrm{S}}+e U\right) \mathrm{d} U \\
& D_{\mathrm{T}}^{\mathrm{MAGN}}(x, y, z, V) \\
& =-\varepsilon^{2} \frac{e^{2}}{h} \sum_{a} \int_{0}^{V} \mathrm{e}^{-2 \kappa\left(E_{\mathrm{F}}^{\mathrm{S}}+e U, V\right) d_{a}(x, y, z)} \\
& \quad \times \frac{\partial \boldsymbol{m}_{\mathrm{T}}}{\partial U}\left(E_{\mathrm{F}}^{\mathrm{T}}+e U-e V\right) \boldsymbol{m}_{\mathrm{S}}^{a}\left(E_{\mathrm{F}}^{\mathrm{S}}+e U\right) \mathrm{d} U
\end{aligned}
$$

Thus, all components of the differential conductance are formulated in spin-polarized tunnel junctions within the 3D WKB framework using first principles electronic structure of the sample and the tip. One can calculate these differential conductance contributions and related quantities at $(x, y, z)$ grid points of a $3 \mathrm{D}$ fine grid in a finite box above the surface, for a sketch see Fig. 1. More details about extracting spectroscopic information from the simulated data can be found in Sections 2.4.3 and 2.4.4.

2.3 Orbital dependent tunneling within the $3 \mathrm{D}$ WKB approach

In this section the topographic part of the tunneling properties are considered only, but the described procedure can be extended to magnetic systems as well. So far the independent orbital approximation $[12,13,90]$ has been used for describing the tunneling transmission, see Eq. (12). Next, this tunneling model is extended by taking advantage of the orbital decomposition of the electronic structure data and the real space shape of the electron orbitals. The PDOS of the sample surface atoms and the tip apex can be decomposed according to orbital symmetry, i.e., real spherical harmonics, $\alpha, \beta \in\left\{s, p_{y}, p_{z}, p_{x}, d_{x y}, d_{y z}, d_{3 z^{2}-r^{2}}, d_{x z}, d_{x^{2}-y^{2}}\right\}$, as

$$
\begin{aligned}
& n_{\mathrm{S}}^{a}(E)=\sum_{\alpha} n_{\mathrm{S} \alpha}^{a}(E) \\
& n_{\mathrm{T}}(E)=\sum_{\beta} n_{\mathrm{T} \beta}(E)
\end{aligned}
$$

Similar decomposition of the Green functions has been employed in the linear combination of atomic orbitals (LCAO) scheme by Refs. [24, 29]. Assuming such an orbital decomposition, the virtual differential conductance 
can be generalized as

$$
\begin{aligned}
& \frac{\mathrm{d} I}{\mathrm{~d} U}(x, y, z, U, V) \\
& =\varepsilon^{2} \frac{e^{2}}{h} \sum_{a} \sum_{\alpha, \beta} T_{\alpha \beta}\left(E_{\mathrm{F}}^{\mathrm{S}}+e U, V, d_{a}(x, y, z)\right) \\
& \quad \times n_{\mathrm{T} \beta}\left(E_{\mathrm{F}}^{\mathrm{T}}+e U-e V\right) n_{\mathrm{S} \alpha}^{a}\left(E_{\mathrm{F}}^{\mathrm{S}}+e U\right)
\end{aligned}
$$

where, in addition to the atomic superposition (sum over a), each $\alpha \leftrightarrow \beta$ transition with an orbital dependent tunneling transmission is summed up: $T_{\alpha \beta}\left(E, V, d_{a}\right)$ gives the electron tunneling probability at energy $E$ from the tip apex $\beta$ orbital to the $\alpha$ orbital of the ath surface atom at positive bias voltage $(V>0)$, and from the $\alpha$ orbital of the $a$ th surface atom to the tip apex $\beta$ orbital at negative bias $(V<0) . T_{\alpha \beta}$ can be defined in different ways based on physical arguments. Ref. [91] considers the following form,

$$
T_{\alpha \beta}\left(E_{\mathrm{F}}^{\mathrm{S}}+e U, V, d_{a}\right)=\mathrm{e}^{-2 \kappa\left(E_{\mathrm{F}}^{\mathrm{S}}+e U, V\right) d_{a}} t_{\alpha \beta}\left(\vartheta_{a}, \varphi_{a}\right)
$$

for each surface atom $\leftrightarrow$ tip apex 1D electron transition. Here, the energy and bias dependent part corresponds to the spherical exponential decay considered in Eq. (12), and is independent of the orbital symmetry. This is multiplied by an orbital dependent expression $t_{\alpha \beta}$, which depends on the spatial arrangement of the sample atoms relative to the tip apex and all the orbital shapes involved in the tunneling. The angular dependence on $\vartheta_{a}$ and $\varphi_{a}$ comes into play in the following way: Let us consider one transition between surface atom $a$ and the tip apex along the line connecting the two atoms. A particular geometry is shown in Fig. 2. For brevity, the $a$ notation of the surface atom is omitted. For both atoms a local coordinate system can be set up, and the angular dependence of the atomic orbital wave functions $\chi(\vartheta, \varphi)$ are defined in the corresponding coordinate system, as summarized in Table 2. $\boldsymbol{R}_{\text {TIP }}-\boldsymbol{R}_{a}$ defines a vector pointing from the surface atom toward the tip apex, and it can be represented by the $\left(d_{a}, \vartheta_{a}, \varphi_{a}\right)$ coordinates in the spherical coordinate system centered on the $a$ th surface atom. Taking the opposite connecting vector from the tip apex toward the surface atom, its coordinates are $\left(d_{a}, \pi-\vartheta_{a}, \pi+\varphi_{a}\right)$ in the spherical coordinate system centered on the tip apex. According to Fig. 2, $d_{a}, \vartheta_{a}$, and $\varphi_{a}$ can be calculated as

$$
\begin{aligned}
d_{a} & =\sqrt{\left(x-x_{a}\right)^{2}+\left(y-y_{a}\right)^{2}+\left(z-z_{a}\right)^{2}} \\
\vartheta_{a} & =\arccos \frac{z-z_{a}}{d_{a}} \\
\varphi_{a} & =\arccos \frac{x-x_{a}}{d_{a} \sin \vartheta_{a}}
\end{aligned}
$$

using the global coordinates $\boldsymbol{R}_{\mathrm{TIP}}=(x, y, z)$ and $\boldsymbol{R}_{a}=$ $\left(x_{a}, y_{a}, z_{a}\right)$. Considering the above expressions, $t_{\alpha \beta}$ accounts for the modification of the perfect spherical exponential decay along the connecting line through the angular dependence of the atomic orbitals as

$$
t_{\alpha \beta}\left(\vartheta_{a}, \varphi_{a}\right)=\left[\chi_{\alpha}\left(\vartheta_{a}, \varphi_{a}\right)\right]^{2} \times\left[\chi_{\beta}\left(\pi-\vartheta_{a}, \pi+\varphi_{a}\right)\right]^{2}
$$

where $\chi_{\alpha, \beta}(\vartheta, \varphi)$ are the real spherical harmonics summarized in Table 2 . They were chosen in such a way that $0 \leqslant t_{\alpha \beta} \leqslant 1$. This factor takes the effect of the directional tunneling between real space orbitals into account. The physical motivation is the angular dependence of the electron density of the orbitals in the given tunneling direction, which modifies the tunneling transmission. The maximum $t=1$ is obtained if the angular distributions of the electron density according to the given orbital symmetries $(\alpha, \beta)$ around both the sample surface atom and the tip apex have maxima along the line of the tunneling direction. This is always the case for $s-s$ type of tunneling irrespective of the relative position of the tip apex and sample surface atoms, i.e., one can observe perfect spherical exponential decay between tip and sample $s$ orbitals. In some particular geometries $t=1$ can be obtained even for other type of orbitals, e.g., if the tip apex is precisely above surface atom $a$, i.e., $\vartheta_{a}=0$, then $t_{\alpha \beta}\left(\vartheta_{a}=0, \varphi_{a}\right)=1$ for all of the following combinations: $\alpha, \beta \in\left\{s, p_{z}, d_{3 z^{2}-r^{2}}\right\}$. On the other hand, if the tip apex is above surface atom $a$ then orbitals with nodal planes orthogonal to the surface have zero contribution to the tunneling from this particular surface atom, i.e., a reduced effective tunneling transmission is obtained [22]. Note that the independent orbital approximation corresponds to $t_{\alpha \beta}=1$ for all $\alpha \leftrightarrow \beta$ transitions, i.e., the same tunneling transmission is assumed between all orbitals. Within this orbital dependent tunneling approach ideal tip models with particular orbital symmetries can be considered, i.e., $\beta_{0}$ orbital symmetry corresponds to the choice of $n_{\mathrm{T} \beta_{0}}=1(\mathrm{eV})^{-1}$ and $n_{\mathrm{T}\left(\beta \neq \beta_{0}\right)}=0$. More realistic tips can be obtained by explicitly calculating the

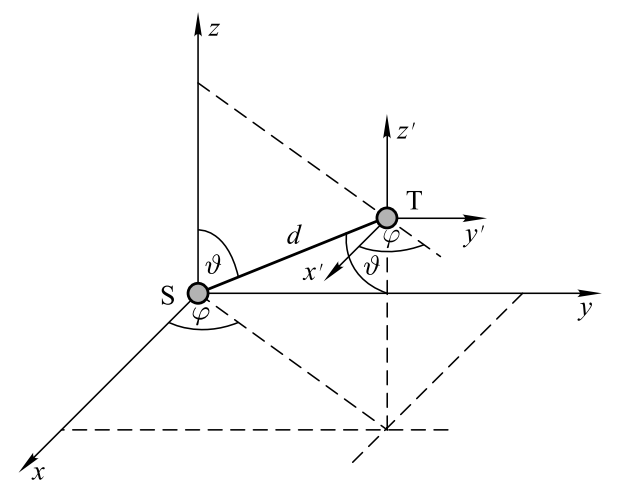

Fig. 2 Geometry of a general tip apex-sample atom setup. Reproduced from Ref. [91]. 
Table 2 Real space orbitals, their indices used in the present paper, their definition from spherical harmonics $Y_{l}^{m}(\vartheta, \varphi)$, and the angular dependence of their wave functions $\chi(\vartheta, \varphi)$. Note that $\vartheta$ and $\varphi$ are the usual polar and azimuthal angles, respectively, in the spherical coordinate system centered on the corresponding (tip or sample) atom. This Table is taken from Ref. [91].

\begin{tabular}{cccc}
\hline Orbital & Index & Definition & $\chi(\vartheta, \varphi)$ \\
\hline$s$ & 1 & $Y_{0}^{0}$ & 1 \\
$p_{y}$ & 2 & $Y_{1}^{1}-Y_{1}^{-1}$ & $\sin \vartheta \sin \varphi$ \\
$p_{z}$ & 3 & $Y_{1}^{0}$ & $\cos \vartheta$ \\
$p_{x}$ & 4 & $Y_{1}^{1}+Y_{1}^{-1}$ & $\sin \vartheta \cos \varphi$ \\
$d_{x y}$ & 5 & $Y_{2}^{2}-Y_{2}^{-2}$ & $\sin ^{2} \vartheta \sin (2 \varphi)$ \\
$d_{y z}$ & 6 & $Y_{2}^{1}-Y_{2}^{-1}$ & $\sin (2 \vartheta) \sin \varphi$ \\
$d_{3 z^{2}-r^{2}}$ & 7 & $Y_{2}^{0}$ & $\frac{1}{2}\left(3 \cos ^{2} \vartheta-1\right)$ \\
$d_{x z}$ & 8 & $Y_{2}^{1}+Y_{2}^{-1}$ & $\sin (2 \vartheta) \cos \varphi$ \\
$d_{x^{2}-y^{2}}$ & 9 & $Y_{2}^{2}+Y_{2}^{-2}$ & $\sin ^{2} \vartheta \cos (2 \varphi)$ \\
\hline
\end{tabular}

orbital decomposition of the tip apex PDOS in model tip geometries.

The presented theory is thus an extension of the 3D WKB STM/STS approach considering tunneling between directional orbitals. The tunneling current and the differential conductance can be calculated at the tip position $\boldsymbol{R}_{\text {TIP }}(x, y, z)$ and at bias voltage $V$ as the sum of all $\alpha \leftrightarrow \beta$ transitions between real space orbitals,

$$
\begin{aligned}
& I(x, y, z, V)=\sum_{\alpha, \beta} I_{\alpha \beta}(x, y, z, V) \\
& \frac{\mathrm{d} I}{\mathrm{~d} V}(x, y, z, V)=\sum_{\alpha, \beta} \frac{\mathrm{d} I_{\alpha \beta}}{\mathrm{d} V}(x, y, z, V)
\end{aligned}
$$

respectively, with

$$
\begin{aligned}
& I_{\alpha \beta}(x, y, z, V) \\
& =\varepsilon^{2} \frac{e^{2}}{h} \sum_{a} \int_{0}^{V} T_{\alpha \beta}\left(E_{\mathrm{F}}^{\mathrm{S}}+e U, V, d_{a}(x, y, z)\right) \\
& \times n_{\mathrm{T} \beta}\left(E_{\mathrm{F}}^{\mathrm{T}}+e U-e V\right) n_{\mathrm{S} \alpha}^{a}\left(E_{\mathrm{F}}^{\mathrm{S}}+e U\right) \mathrm{d} U \\
& \frac{\mathrm{d} I_{\alpha \beta}}{\mathrm{d} V}(x, y, z, V) \\
& =\varepsilon^{2} \frac{e^{2}}{h}\left\{\sum_{a} T_{\alpha \beta}\left(E_{\mathrm{F}}^{\mathrm{S}}+e V, V, d_{a}(x, y, z)\right)\right. \\
& \times n_{\mathrm{T} \beta}\left(E_{\mathrm{F}}^{\mathrm{T}}\right) n_{\mathrm{S} \alpha}^{a}\left(E_{\mathrm{F}}^{\mathrm{S}}+e V\right) \\
& +\sum_{a} \int_{0}^{V} \frac{\partial T_{\alpha \beta}}{\partial V}\left(E_{\mathrm{F}}^{\mathrm{S}}+e U, V, d_{a}(x, y, z)\right) \\
& \times n_{\mathrm{T} \beta}\left(E_{\mathrm{F}}^{\mathrm{T}}+e U-e V\right) n_{\mathrm{S} \alpha}^{a}\left(E_{\mathrm{F}}^{\mathrm{S}}+e U\right) \mathrm{d} U \\
& -\sum_{a} \int_{0}^{V} T_{\alpha \beta}\left(E_{\mathrm{F}}^{\mathrm{S}}+e U, V, d_{a}(x, y, z)\right) \\
& \left.\times \frac{\partial n_{\mathrm{T} \beta}}{\partial U}\left(E_{\mathrm{F}}^{\mathrm{T}}+e U-e V\right) n_{\mathrm{S} \alpha}^{a}\left(E_{\mathrm{F}}^{\mathrm{S}}+e U\right) \mathrm{d} U\right\}
\end{aligned}
$$

This decomposition enables the analysis of the orbital contributions to the total tunneling current and to the differential conductance. It is important to note that while the formulation of Chen's derivative rule [21] is inspired by the Tersoff-Hamann model, and calculates the tunneling transmission as the absolute value square of the tunneling matrix element that is proportional to the sample wave function derivative with respect to the real space coordinate corresponding to the given tip orbital symmetry $(\beta)$, the hereby presented transmission function also depends on the sample surface atoms' orbital symmetry $(\alpha)$. Moreover, the electronic structure of the tip apex is included in this orbital dependent tunneling theory via the PDOS.

Note that the presented orbital-dependent method can also be applied to magnetic systems taking into account the orbital-decomposed magnetization PDOS of the tip and sample together with the orbital dependent tunneling transmission in Eq. (55). As it was pointed out by Ferriani et al. [76], the spin polarization in the vacuum can have an opposite sign than within the tip apex atom, and this sign change is also accompanied by different dominating orbital characters. Thus, the consideration of an orbital dependent tunneling transmission might be a better model for describing electron transport through a magnetic tunnel junction.

\subsection{Tunneling properties}

From the calculated current and differential conductances, a wide spectrum of information characteristic for the specific surface-tip combination can be obtained, and also generally important findings can be derived. It has to be noted that the presented SP-STM and SP-STS methods can also be applied to study nonmagnetic systems, where all magnetic contributions equal zero, and the corresponding topographic STM images and STS spectra can be simulated. In this case, the magnetic asymmetry is also zero.

\subsubsection{Current}

From the obtained 3D tunneling current maps, $I(x, y, z, V, T)$, data can be extracted that are directly comparable to experiments. In particular, current values can be shown in arbitrary $z=Z_{C}=$ const planes or constant-value contours can be defined. The first option corresponds to the constant height mode, $I\left(x, y, Z_{C}=\right.$ const, $V, T)$, while the second to the constant current mode of SP-STM, $I(x, y, z, V, T)=I_{C}=$ const. From the latter, a $2 \mathrm{D}$ tip position map, called the height profile, $z\left(x, y, V, T, I_{C}\right)$ can be extracted using logarithmic interpolation between grid points $z_{1}<z_{2}$, if $I\left(x, y, z_{1}, V, T\right)>$ $I_{C}>I\left(x, y, z_{2}, V, T\right)$, in the following way [56], 


$$
\begin{aligned}
& z\left(x, y, V, T, I_{C}\right) \\
& \quad=z_{1}+\Delta z \frac{\ln I_{C}-\ln I\left(x, y, z_{1}, V, T\right)}{\ln I\left(x, y, z_{2}, V, T\right)-\ln I\left(x, y, z_{1}, V, T\right)}
\end{aligned}
$$

where $\Delta z=z_{2}-z_{1}=z_{i+1}-z_{i}=0.1$ a.u. $(\approx 0.053 \AA)$ gives a fine resolution in the surface normal $z$-direction. A constant current contour is demonstrated inside the finite box above a surface in Fig. 1. Alternatively, if $I_{C}$ has such a value that is not contained in the considered finite box above the surface, i.e., if $I_{C}<I\left(z_{\max }\right)$, then

$$
\begin{aligned}
& z\left(x, y, V, T, I_{C}\right)=z_{\max }+\Delta z \\
& \quad \times \frac{\ln I_{C}-\ln I\left(x, y, z_{\max }, V, T\right)}{\ln I\left(x, y, z_{\max }, V, T\right)-\ln I\left(x, y, z_{\max -1}, V, T\right)}
\end{aligned}
$$

Surface corrugation can be determined from this $z(x, y)$ map. Note that the total current contains both topographic and magnetic contributions, therefore $z(x, y)$ will be the simulated SP-STM image [56, 90]. In the presented model its bias and temperature dependence is considered as well. In periodic magnetic systems the magnetic unit cell can be identified in the simulated image [15].

$I_{\mathrm{TOPO}}$ and $I_{\mathrm{MAGN}}$ in Eqs. (37) and (38), respectively, can be analyzed separately using the same way as described above, and they can be related to SP-STM experiments using the differential magnetic mode [43]. From the non-magnetic height profile, $z\left(x, y, V, T, I_{\mathrm{TOPO}}=\right.$ const), the surface topography can be calculated, and in periodic systems the chemical unit cell is revealed in the simulated image.

\subsubsection{Contrast}

The contrast between two lateral surface positions is defined as their apparent height difference on a constant current contour.

On a nonmagnetic constant current STM image the contrast between atoms $A$ and $B$ on the surface at the average tip-sample distance $z_{1}$ and bias voltage $V$ is $[90,99]$

$$
\Delta z_{\text {nonmagn }}^{A B}\left(z_{1}, V\right)=-\frac{\Delta I^{A B}\left(z_{1}, V\right)}{\partial I^{a v} / \partial z\left(z_{1}, V\right)}
$$

where $\Delta I^{A B}\left(z_{1}, V\right)$ is the current difference above atoms $A$ and $B$ at the tip-sample distance $z_{1}$, and $I^{a v}(z, V)$ is a laterally averaged current over the surface chemical unit cell at a tip-sample distance of $z$. Since it is a tunneling current, it decays exponentially as $z$ increases [99].

Next, the above relation between constant current and constant height STM images is adopted to the SP-STM scenario. Similarly to Eq. (1), the total contrast can also be decomposed as the sum of topographic and magnetic contributions,

$$
\begin{aligned}
& \Delta z^{A B}\left(z_{1}, V\right)=\Delta z_{\mathrm{TOPO}}^{A B}\left(z_{1}, V\right)+\Delta z_{\mathrm{MAGN}}^{A B}\left(z_{1}, V\right) \\
& =-\frac{\Delta I_{\mathrm{TOPO}}^{A B}\left(z_{1}, V\right)}{\partial I_{\mathrm{TOTAL}}^{a v} / \partial z\left(z_{1}, V\right)}-\frac{\Delta I_{\mathrm{MAGN}}^{A B}\left(z_{1}, V\right)}{\partial I_{\mathrm{TOTAL}}^{a v} / \partial z\left(z_{1}, V\right)}
\end{aligned}
$$

Here, $\Delta I_{\mathrm{TOPO}}^{A B}$ and $\Delta I_{\mathrm{MAGN}}^{A B}$ are the respective topographic and magnetic current differences above atoms $A$ and $B . I_{\text {TOTAL }}^{a v}$ has to be calculated by laterally averaging the total current over the surface (chemical and magnetic) supercell at a constant tip-sample distance,

$$
I_{\text {TOTAL }}^{a v}(z, V)=\frac{1}{N_{x} N_{y}} \sum_{i=1}^{N_{x}} \sum_{j=1}^{N_{y}} I_{\text {TOTAL }}\left(x_{i}, y_{j}, z, V\right)
$$

where $N_{x}$ and $N_{y}$ denote the number of grid points in the lateral $x$ and $y$ directions, respectively. Again, $I_{\text {TOTAL }}^{a v}(z, V)$ is expected to decay exponentially as $z$ increases.

In the following, let us focus on the magnetic contrast only. Therefore, let us assume an atomically flat sample surface consisting of chemically equivalent but magnetically inequivalent atoms. In this case the topographic contrast between any two surface atoms disappears since $\Delta I_{\mathrm{TOPO}}^{A B}=0$. Hence, the total contrast between surface atoms is the magnetic contrast, $\Delta z^{A B}\left(z_{1}, V\right)=$ $\Delta z_{\mathrm{MAGN}}^{A B}\left(z_{1}, V\right)$.

Since the calculation of the contrast requires the $z$ derivative of the exponentially decaying laterally averaged total current in Eq. (68), a full scanning of the surface magnetic unit cell at two constant heights is necessary. The measurement time of this is comparable to record the constant current contour above the same scan area, thus there is no advantage of using Eq. (67) for the contrast estimation. It is advantageous to avoid any scanning above the surface but still predict the magnetic contrast between two surface atoms on a constant current contour. Therefore, the denominator in Eq. (67) needs to be reconsidered, and it is allowed to contain current quantities above the two lateral sites $A$ and $B$ only.

A motivation for constructing the magnetic contrast formula is suggested by the following analogy at constant current condition: In a nonmagnetic STM image the modulation due to the surface atoms is superimposed on the average tip-sample distance, whereas in an SPSTM image of a complex magnetic surface the magnetic modulation is superimposed on the topographic image. Therefore, taking Eq. (66) and generalizing to the SPSTM case, the small lateral variation of the current due to the magnetic modulation plays the role of the numerator, i.e., $\Delta I^{A B} \rightarrow \Delta I_{\mathrm{MAGN}}^{A B}$, and the topographic current takes the role of the average current in the denominator, i.e., $I^{a v} \rightarrow I_{\mathrm{TOPO}}$. This is a fortunate choice 
since the topographic currents above all surface atoms are the same due to the assumed chemical equivalence, $I_{\mathrm{TOPO}}^{A}(z, V)=I_{\mathrm{TOPO}}^{B}(z, V)$. Following this, a magnetic contrast between atoms $A$ and $B$ on the surface at the tip-sample distance $z_{1}$ and bias voltage $V$ can be defined as

$$
\begin{gathered}
\Delta z_{I}^{A B}\left(z_{1}, V\right)=-\frac{\Delta I_{\mathrm{MAGN}}^{A B}\left(z_{1}, V\right)}{\partial I_{\mathrm{TOPO}}^{A} / \partial z\left(z_{1}, V\right)} \\
=\frac{I_{\mathrm{MAGN}}^{A}\left(z_{1}, V\right)-I_{\mathrm{MAGN}}^{B}\left(z_{1}, V\right)}{2 \kappa_{\mathrm{TOPO}}^{A}(V) I_{\mathrm{TOPO}}^{A}\left(z_{1}, V\right)}
\end{gathered}
$$

where the exponentially decaying character of the topographic tunneling current is used,

$$
I_{\mathrm{TOPO}}^{A}(z, V)=I_{\mathrm{TOPO}}^{A 0}(V) \mathrm{e}^{-2 \kappa_{\mathrm{TOPO}}^{A}(V) z}
$$

An even more straightforward idea is to approximate the lateral average of the total tunneling current over the magnetic unit cell as the average of the currents measured above the $A$ and $B$ sites, which is still supposed to decay exponentially with respect to $z$ [57],

$$
\begin{aligned}
& I_{\text {TOTAL }}^{a v \cdot A B}(z, V)=\frac{I_{\text {TOTAL }}^{A}(z, V)+I_{\text {TOTAL }}^{B}(z, V)}{2} \\
& \quad=I_{\text {TOTAL }}^{a v \cdot A B 0}(V) \mathrm{e}^{-2 \kappa_{\text {TOTAL }}^{a v \cdot A B}(V) z}
\end{aligned}
$$

Using this quantity, another magnetic contrast between atoms $A$ and $B$ on the surface at the tip-sample distance $z_{1}$ and bias voltage $V$ can be defined as

$$
\begin{gathered}
\Delta z_{I I}^{A B}\left(z_{1}, V\right)=-\frac{\Delta I_{\mathrm{MAGN}}^{A B}\left(z_{1}, V\right)}{\partial I_{\mathrm{TOTAL}}^{a \cdot A B} / \partial z\left(z_{1}, V\right)} \\
=\frac{I_{\mathrm{MAGN}}^{A}\left(z_{1}, V\right)-I_{\mathrm{MAGN}}^{B}\left(z_{1}, V\right)}{2 \kappa_{\mathrm{TOTAL}}^{a v . A B}(V) I_{\mathrm{TOTAL}}^{a v . A B}\left(z_{1}, V\right)}
\end{gathered}
$$

Assuming that $\Delta z^{A B}\left(z_{1}, V\right)$ and $\Delta z^{A B}\left(z_{2}, V\right)$ magnetic contrasts are available at $z_{1} \neq z_{2}$ tip-sample distances, employing either Eq. (69) or Eq. (72), and further assuming an exponentially decaying magnitude of the contrast with increasing tip-sample distance, the bias dependent magnetic contrast between atoms $A$ and $B$ at an arbitrary tip-sample distance $z$ in the tunneling regime can be estimated as

$$
\begin{aligned}
\Delta z^{A B}(z, V)= & \operatorname{sgn}\left(\Delta z^{A B}\left(z_{1}, V\right)\right)\left|\Delta z^{A B}\left(z_{1}, V\right)\right|^{\frac{z_{2}-z}{z_{2}-z_{1}}} \\
& \times\left|\Delta z^{A B}\left(z_{2}, V\right)\right|^{\frac{z-z_{1}}{z_{2}-z_{1}}}
\end{aligned}
$$

The derivation of this formula is reported in the Appendix of Ref. [57].

\subsubsection{Differential conductance}

From the 3D differential conductance map, data can be extracted that are directly comparable to experiments. For example, a single point spectrum corresponds to a fixed $\left(x_{0}, y_{0}, z_{0}\right)$ tip position, and $2 \mathrm{D}$ spectra can also be obtained, where the image resolution is determined by the density of $(x, y)$ grid points. There are usually two ways to define a 2D differential conductance map [42]. The first method fixes the tip height at $z=Z_{\text {stab }}=$ const and scans the surface, $\mathrm{d} I / \mathrm{d} V\left(x, y, Z_{\text {stab }}, V\right)$. The second option measures $\mathrm{d} I / \mathrm{d} V$ on a constant current contour, $I_{\text {TOTAL }}=I_{\text {stab }}=$ const, which is the widely used method in experiments. Simulation of this can be done in two steps: First, the 3D current map with the given bias voltage $V_{\text {stab }}$ is calculated, and at the second step the height profile of a constant current contour, $z\left(x, y, V_{\text {stab }}, I_{\text {stab }}\right)$, is determined by using logarithmic interpolation, see Eqs. (64) and (65). $V_{\text {stab }}$ and $I_{\text {stab }}$ are the tunneling parameters, and they stabilize the tip position at the height of $z\left(x, y, V_{\text {stab }}, I_{\mathrm{TOTAL}}=I_{\text {stab }}\right)$ above the $(x, y)$ sample surface point. The $2 \mathrm{D}$ differential conductance map on the constant current contour is then given by $\mathrm{d} I / \mathrm{d} V\left(x, y, z\left(x, y, V_{\text {stab }}, I_{\mathrm{TOTAL}}=I_{\text {stab }}\right), V\right)$, where the $V$-dependence is obtained by sweeping the bias voltage range using a lock-in technique in experiments [42].

Recently, experimental efforts have been made to extract the TOPO component of the tunneling current [100], and measure spectroscopic data on such constant current contours, i.e., at $I_{\mathrm{TOPO}}=$ const [101]. According to Ref. [42], a constant tunneling transmission enables an easier interpretation of measured 2D spectroscopic data. It seems to be straightforward that a constant TOPO current contour is closer to this constant tunneling transmission criterion than a constant TOTAL current contour due to the appearance of spin dependent effects in the latter one. On the other hand, the calculation of any current contour is simple within the presented $3 \mathrm{D}$ WKB approach [56]. Since the $I_{\mathrm{TOPO}}=$ const experimental method is not routinely available at the moment, we restrict ourselves to consider the $I_{\mathrm{TOTAL}}=$ const contours when calculating the $2 \mathrm{D}$ differential conductance maps, and examples will be shown in Section 3.8.

The interpretation of $2 \mathrm{D} \mathrm{d} I / \mathrm{d} V$ maps has to be taken with care even on nonmagnetic surfaces. For example, very recently Krenner et al. [102] discussed the physical reasons of the difference between $2 \mathrm{D} \mathrm{d} I / \mathrm{d} V$ maps measured at constant-current and open-feedback-loop conditions on strongly corrugated surfaces.

\subsubsection{Magnetic asymmetry}

By simulating differential conductance spectra above a magnetic surface with parallel (P) and antiparallel (AP) tip magnetization directions with respect to a pre-defined direction (usually the magnetization direction of a chosen surface atom is taken), the so-called magnetic asymmetry can be defined [75]. In the presented case this quan- 
tity can be calculated at all considered positions of the tip apex atom, i.e., at all $(x, y, z)$ grid points within the finite box above the surface:

$$
\begin{aligned}
& A(x, y, z, V) \\
& \quad=\frac{\mathrm{d} I^{P} / \mathrm{d} V(x, y, z, V)-\mathrm{d} I^{A P} / \mathrm{d} V(x, y, z, V)}{\mathrm{d} I^{P} / \mathrm{d} V(x, y, z, V)+\mathrm{d} I^{A P} / \mathrm{d} V(x, y, z, V)}
\end{aligned}
$$

From this, the magnetic asymmetry can similarly be calculated on appropriate constant current contours as described in the previous section. Using Eq. (40), and the fact that the magnetic contribution for the AP tip magnetization direction $\mathrm{d} I_{\mathrm{MAGN}}^{A P} / \mathrm{d} V$ equals $-\mathrm{d} I_{\text {MAGN }}^{P} / \mathrm{d} V$, since the tip magnetization PDOS vector $\boldsymbol{m}_{\mathrm{T}}(E)$ changes sign at all energies compared to the $\mathrm{P}$ tip magnetization direction, the differential conductances take the following form:

$$
\begin{aligned}
\mathrm{d} I^{P} / \mathrm{d} V(x, y, z, V)= & \mathrm{d} I_{\mathrm{TOPO}} / \mathrm{d} V(x, y, z, V) \\
& +\mathrm{d} I_{\text {MAGN }}^{P} / \mathrm{d} V(x, y, z, V) \\
\mathrm{d} I^{A P} / \mathrm{d} V(x, y, z, V)= & \mathrm{d} I_{\mathrm{TOPO}} / \mathrm{d} V(x, y, z, V) \\
& -\mathrm{d} I_{\text {MAGN }}^{P} / \mathrm{d} V(x, y, z, V)
\end{aligned}
$$

Thus, the magnetic asymmetry can be expressed as the fraction of the MAGN and TOPO differential conductances from Eqs. (42) and (43) as

$$
\begin{aligned}
A^{\mathrm{d} I / \mathrm{d} V}(x, y, z, V) & =\frac{\mathrm{d} I_{\mathrm{MAGN}}^{P} / \mathrm{d} V(x, y, z, V)}{\mathrm{d} I_{\mathrm{TOPO}} / \mathrm{d} V(x, y, z, V)} \\
& =\frac{\mathrm{d} I_{\mathrm{MAGN}}^{P} / \mathrm{d} U(x, y, z, V, V)+B_{\mathrm{MAGN}}^{P}(x, y, z, V)+D_{\mathrm{T}}^{\mathrm{MAGN}, P}(x, y, z, V)}{\mathrm{d} I_{\mathrm{TOPO}} / \mathrm{d} U(x, y, z, V, V)+B_{\mathrm{TOPO}}(x, y, z, V)+D_{\mathrm{T}}^{\mathrm{TOPO}}(x, y, z, V)}
\end{aligned}
$$

This is the correct magnetic asymmetry expression based on the physical differential conductances that can be obtained from experiments. However, a magnetic asymmetry can similarly be defined taking the virtual differential conductances from Eqs. (31) and (32):

$$
A^{\mathrm{d} I / \mathrm{d} U}(x, y, z, V)=\frac{\mathrm{d} I_{\mathrm{MAGN}}^{P} / \mathrm{d} U(x, y, z, V, V)}{\mathrm{d} I_{\mathrm{TOPO}} / \mathrm{d} U(x, y, z, V, V)}
$$

This is an important quantity since it is related to the vacuum spin polarization of the sample in a simple way $[75]$ :

$$
\begin{aligned}
A^{\mathrm{d} I / \mathrm{d} U}(x, y, z, V) & =\boldsymbol{P}_{\mathrm{T}}\left(E_{\mathrm{F}}^{\mathrm{T}}\right) \boldsymbol{P}_{\mathrm{S}}\left(x, y, z, E_{\mathrm{F}}^{\mathrm{S}}+e V\right) \\
& =E S P(x, y, z, V)
\end{aligned}
$$

i.e., $A^{\mathrm{d} I / \mathrm{d} U}(x, y, z, V)$ is the effective spin polarization (ESP): the scalar product of the tip spin polarization vector at its Fermi level, $\boldsymbol{P}_{\mathrm{T}}\left(E_{\mathrm{F}}^{\mathrm{T}}\right)$, and the vacuum spin polarization vector of the sample at $\boldsymbol{R}_{\mathrm{TIP}}(x, y, z), \mathrm{eV}$ above the sample Fermi level, $\boldsymbol{P}_{\mathrm{S}}\left(x, y, z, E_{\mathrm{F}}^{\mathrm{S}}+e V\right)$. Following above, it is clear that the determination of the sample spin polarization from experimentally measured spectra is not straightforward since the experimentally accessible magnetic asymmetry according to the equivalent expressions Eq. (74) and Eq. (76) contains the background and tip-derivative terms as well. On the other hand, one can easily calculate $\operatorname{ESP}(x, y, z, V)$ within the $3 \mathrm{D}$ WKB method, and extract $2 \mathrm{D}$ contours on a constant current contour, see Section 3.8. There are even more possibilities to define magnetic asymmetries, by adding the background terms in Eqs. (48) and (49), or the tipderivative terms in Eqs. (50) and (51) to the corresponding virtual differential conductance and then performing the division:

$$
\begin{aligned}
& A^{\mathrm{d} I / \mathrm{d} U+B}(x, y, z, V) \\
& =\frac{\mathrm{d} I_{\mathrm{MAGN}}^{P} / \mathrm{d} U(x, y, z, V, V)+B_{\mathrm{MAGN}}^{P}(x, y, z, V)}{\mathrm{d} I_{\mathrm{TOPO}} / \mathrm{d} U(x, y, z, V, V)+B_{\mathrm{TOPO}}(x, y, z, V)} \\
& A^{\mathrm{d} I / \mathrm{d} U+D_{\mathrm{T}}}(x, y, z, V) \\
& =\frac{\mathrm{d} I_{\mathrm{MAGN}}^{P} / \mathrm{d} U(x, y, z, V, V)+D_{\mathrm{T}}^{\mathrm{MAGN}, P}(x, y, z, V)}{\mathrm{d} I_{\mathrm{TOPO}} / \mathrm{d} U(x, y, z, V, V)+D_{\mathrm{T}}^{\mathrm{TOPO}}(x, y, z, V)}
\end{aligned}
$$

As $\mathrm{d} I / \mathrm{d} U(V, V)$ is one component of $\mathrm{d} I / \mathrm{d} V(V)$ according to Eq. (41), the comparison of them, and also the magnetic asymmetry expressions in Eqs. (76)-(80) gives useful insights to estimate the error one makes when neglecting the background and tip-derivative components of $\mathrm{d} I / \mathrm{d} V(V)$.

\section{Prototype noncollinear magnetic surface: $\mathrm{Cr} / \mathrm{Ag}(111)$}

In order to demonstrate the capabilities of the described model for simulating SP-STM/STS on complex magnetic surfaces, a sample surface with noncollinear magnetic order is considered $[10,56]$. One monolayer (ML) $\mathrm{Cr}$ on $\mathrm{Ag}(111)$ is a prototype of frustrated hexagonal antiferromagnets [90]. Due to the geometrical frustration of the antiferromagnetic exchange interactions between $\mathrm{Cr}$ spin moments, its magnetic ground state has been determined to be a noncollinear $120^{\circ}$ Néel state [15]. Two possible Néel states with opposite chiralities are considered, that are energetically equivalent only in the absence of spinorbit coupling. 


\subsection{Computational details}

Geometry relaxation and electronic structure calculations were performed based on the Density Functional Theory (DFT) within the Generalized Gradient Approximation (GGA) implemented in the Vienna Ab-initio Simulation Package (VASP) [103-105]. A plane wave basis set for electronic wave function expansion together with the projector augmented wave (PAW) method [106] has been applied, and the exchange-correlation functional is parametrized according to Perdew and Wang (PW91) [107]. For calculating the fully noncollinear electronic structure the VASP code has been used as well [108, 109], with spin-orbit coupling considered. This allows one to determine the Néel state with the energetically favored chirality.

The $\mathrm{Cr} / \operatorname{Ag}(111)$ system was modeled by a slab of a five-layer Ag substrate and one-one monolayer $\mathrm{Cr}$ films on each side, where the surface Cr layers and the first Ag layers underneath have been fully relaxed. After relaxation the $\mathrm{Cr}-\mathrm{Ag}$ interlayer distance was reduced by 9.5\%, and the underneath $\mathrm{Ag}-\mathrm{Ag}$ distance increased by $0.5 \%$ compared to bulk Ag. A separating vacuum region of $14.6 \AA$ width in the surface normal $(z)$ direction has been set up between neighboring supercell slabs. The average electron work function above the $\mathrm{Cr}$ was calculated to be $\phi_{S}=4.47 \mathrm{eV}$ using Eq. (16). An $11 \times 11 \times 1$ Monkhorst-Pack (MP) [110] $k$-point grid was used for calculating the projected electron DOS onto the surface Cr atoms in the $(\sqrt{3} \times \sqrt{3})$ magnetic surface unit cell.

\subsection{Magnetic ground state}

Performing fully noncollinear electronic structure calculations, convergence to two different magnetic Néel states has been obtained [56]. The magnetic surface unit cell with the converged magnetic moment directions are shown in the left part of Fig. 3. Each of the two Néel states can be characterized by a chirality vector, defined as $[53]$

$$
\boldsymbol{K}=\frac{2}{3 \sqrt{3}}\left(\boldsymbol{e}_{\mathrm{S}}^{1} \times \boldsymbol{e}_{\mathrm{S}}^{2}+\boldsymbol{e}_{\mathrm{S}}^{2} \times \boldsymbol{e}_{\mathrm{S}}^{3}+\boldsymbol{e}_{\mathrm{S}}^{3} \times \boldsymbol{e}_{\mathrm{S}}^{1}\right)
$$

Here $\boldsymbol{e}_{\mathrm{S}}^{a}$ denotes the local spin quantization unit vector of the ath $\mathrm{Cr}$ atom. It is defined from the local magnetic moment, $\boldsymbol{M}_{\mathrm{S}}^{a}=\int_{-\infty}^{E_{\mathrm{F}}^{\mathrm{S}}} \boldsymbol{m}_{\mathrm{S}}^{a}(E) \mathrm{d} E$, similarly as in Eq. (23), i.e., $\boldsymbol{e}_{\mathrm{S}}^{a}=\boldsymbol{M}_{\mathrm{S}}^{a} /\left|\boldsymbol{M}_{\mathrm{S}}^{a}\right|$. The magnitude of the magnetic moments of the $\mathrm{Cr}$ surface atoms are $3.73 \mu_{B}$, with a tiny out-of-plane component, that is neglected when defining the chirality vectors. Thus, in the first row of Fig. $3, \boldsymbol{e}_{\mathrm{S}}^{1}=(1 / 2, \sqrt{3} / 2,0), \boldsymbol{e}_{\mathrm{S}}^{2}=(1 / 2,-\sqrt{3} / 2,0)$, and $e_{\mathrm{S}}^{3}=(-1,0,0)$. This corresponds to the chirality vector $\boldsymbol{K}=(0,0,-1)$ or simply $K_{z}=-1$. Similarly, in the second row of Fig. $3, \boldsymbol{e}_{\mathrm{S}}^{1}=(1 / 2,-\sqrt{3} / 2,0)$, $\boldsymbol{e}_{\mathrm{S}}^{2}=(1 / 2, \sqrt{3} / 2,0)$, and $\boldsymbol{e}_{\mathrm{S}}^{3}=(-1,0,0)$ correspond to $K_{z}=+1$. Comparing total energies of the two states, $K_{z}=-1$ is found to be energetically favored by 1.1 meV compared to $K_{z}=+1$. This finding is consistent with the magnetic ground state found for a $\mathrm{Cr}$ trimer island on the $\mathrm{Au}(111)$ substrate in Ref. [53], where it was also shown that the Dzyaloshinskii-Moriya interaction is responsible for determining the ground state magnetic chirality. Performing a collinear calculation with spinorbit coupling considered, a ferromagnetic (FM) state with in-plane $\mathrm{Cr}$ atomic magnetic moments of $3.76 \mu_{B}$ is obtained. It turns out that this FM state is $1.04 \mathrm{eV}$ higher in energy than the $K_{z}=-1$ Néel state. The energy difference of $346 \mathrm{meV} /$ (magnetic atom) in favor of the Néel state is in good agreement with results of Ref.

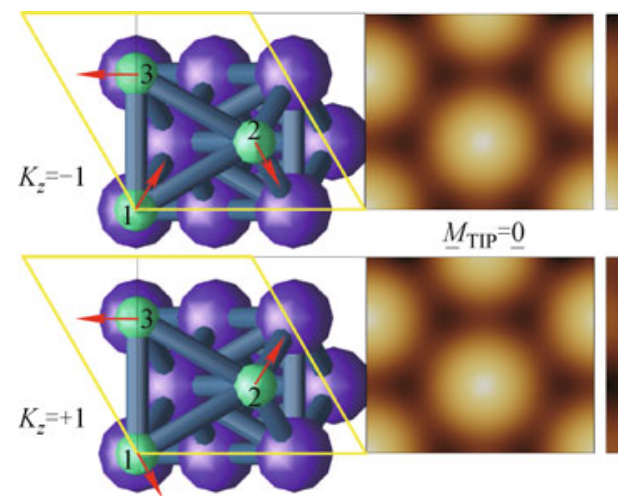

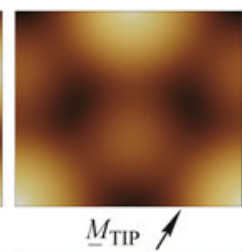

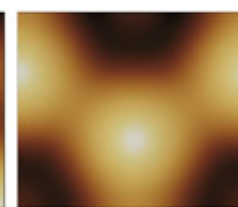

$M_{\mathrm{TIP}}$

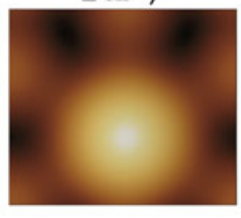

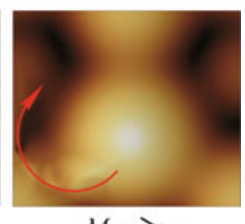

$M_{\mathrm{TIP}} \succ$
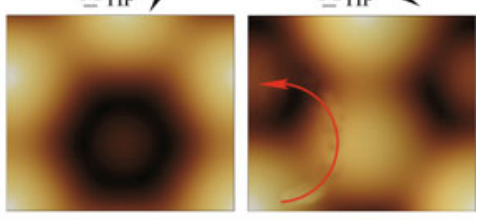

Fig. 3 Surface geometry of $1 \mathrm{ML} \mathrm{Cr}$ on $\mathrm{Ag}(111)$ and simulated SP-STM images at $0 \mathrm{~V}$ bias voltage and $T=4.2 \mathrm{~K}$ depending on the tip magnetization direction $\left(\underline{M}_{\mathrm{TIP}}\right)$ assuming an ideal electronically flat maximally spin-polarized tip. The $\mathrm{Cr}$ and $\mathrm{Ag}$ atoms are denoted by spheres colored by green (medium gray) and purple (dark gray), respectively, and the magnetic moments of individual Cr atoms are indicated by (red) arrows in the left part of the Figure. The Cr atoms are explicitly labeled corresponding to the calculated chirality vector in Eq. (81). In addition, the $(\sqrt{3} \times \sqrt{3})$ magnetic unit cell is drawn by yellow (light gray) color. In the two rows noncollinear Néel states with opposite chiralities and corresponding SP-STM images are shown. In the last column, the decreasing levels of Cr apparent heights are indicated by circular arrows. Reproduced from Ref. [56]. 
[15]. The out-of-plane FM state is $1 \mathrm{meV}$ higher in energy than the in-plane FM state with the same magnitude of magnetic moments.

In the magnetic ground state characterized by $K_{z}=$ -1 , the spin polarization of the surface $\mathrm{Cr}$ atoms is positive with respect to the direction of the corresponding $\mathrm{Cr}$ magnetic moments below $E_{\mathrm{F}}^{\mathrm{S}}+0.54 \mathrm{eV}$, and negative above this energy. More details on the energy dependence of the $\mathrm{Cr}$ spin polarization can be found in Ref. [56].

\subsection{SP-STM detection of magnetic chirality}

In the $\mathrm{Cr} / \mathrm{Ag}(111)$ system the tunneling current has been calculated in a box above the magnetic unit cell containing $153000(34 \times 30 \times 150)$ grid points with a $0.15 \AA$ lateral and $\approx 0.053 \AA$ horizontal resolution. Figure 3 shows simulated constant current SP-STM images for the two Néel states at zero bias voltage and $T=4.2 \mathrm{~K}$, assuming an ideal electronically flat maximally spin-polarized tip with various magnetization directions. These are in qualitatively good agreement with previous simulations $[15,90]$. Using a nonmagnetic tip, all surface Cr atoms appear to be of equal height (one height level), i.e., the surface topography is seen. As the spin polarization of the $\mathrm{Cr}$ atoms at the Fermi energy is positive [56], and the tip spin polarization is set to +1 , the $\mathrm{Cr}$ atom with parallel/antiparallel magnetization direction relative to the tip appears to be higher/lower than the other two Cr atoms, which have the same apparent height due to symmetry (two height levels). Comparing the images, it is clear that a contrast reversal occurs when turning the tip magnetization to opposite direction. By setting the tip magnetization direction perpendicular to a $\mathrm{Cr}$ magnetic moment, a structure with three height levels occurs. This means that all $\mathrm{Cr}$ atoms in the magnetic unit cell have different apparent heights. This is due to the variation of the angles between the local $\mathrm{Cr}$ magnetic moments and the tip magnetization, e.g., for $K_{z}=-1$, $\varphi_{1}=90^{\circ}, \varphi_{2}=30^{\circ}$, and $\varphi_{3}=150^{\circ}$. Determining the chirality of the magnetic structure from experimental SPSTM images is only possible in such a scenario if the tip magnetization direction is not parallel to the magnetic moment of any of the surface atoms. In the example of the $\mathrm{Cr} / \mathrm{Ag}(111)$ system the three apparent height levels follow a different order in the magnetic unit cell corresponding to the different chiralities. The decreasing levels of $\mathrm{Cr}$ apparent heights are indicated by circular arrows in the last column of Fig. 3. Generally, the determining factor for the apparent height of magnetic atoms in zero bias $(V=0 \mathrm{~V})$ measurements is the effective spin polarization (ESP) at the common Fermi level, $P_{\mathrm{T}}\left(E_{\mathrm{F}}^{\mathrm{S}}\right) P_{\mathrm{S}}^{a}\left(E_{\mathrm{F}}^{\mathrm{S}}\right) \cos \xi_{a}\left(E_{\mathrm{F}}^{\mathrm{S}}\right)$, similarly as it was identified as the governing factor for the height of differential tun- neling spectra at particular energies [89]. A positive ESP results in higher tunneling current at a fixed distance above a magnetic surface atom, while the opposite holds for negative ESP. Considering a constant current contour, thus, results in a larger apparent height for the atom with positive, while a smaller height with negative ESP, compared to the topographic heights.

\subsection{Tip effects on the SP-STM images}

In the following the magnetic Néel state with $K_{z}=-1$ chirality is considered since it has been identified as the ground state. By including the energy dependent electronic structure of the sample and the tip into the tunneling model, one can study the bias dependent magnetic contrast and its tip dependence as well. Figure 4 shows simulated SP-STM images for various tip magnetization directions at $-1 \mathrm{~V}, 0 \mathrm{~V}$ and $+1 \mathrm{~V}$ bias voltages assuming an ideal magnetic tip. One finds qualitatively similar images for $-1 \mathrm{~V}$ and $0 \mathrm{~V}$ for the corresponding tip magnetization direction. This means that the $\Delta z_{\mathrm{Cr} 1-\mathrm{Cr} 3}$ magnetic contrast has the same sign at $-1 \mathrm{~V}$ and $0 \mathrm{~V}$. However, the magnitude of $\Delta z_{\mathrm{Cr} 1-\mathrm{Cr} 3}$ at the same $\mathrm{Cr} 1$ apparent height increases at $-1 \mathrm{~V}$ compared to $0 \mathrm{~V}$ for all tip magnetization directions. This can be explained by the integrated $\mathrm{d} I_{\mathrm{MAGN}}^{P}$ contribution, that does not change sign in this bias range, see Ref. [56]. On the other hand, the results show that the magnetic contrast is reversed at $+1 \mathrm{~V}$ compared to the other two studied bias voltages. This contrast reversal is observed for all tip magnetization directions. It is interesting to find that on the image with three height levels, the apparent heights change order in such a way that the image at $+1 \mathrm{~V}$ looks like that the Néel state would have an opposite chirality compared to $0 \mathrm{~V}$ or $-1 \mathrm{~V}$, see the indicated circular arrows in the last row of Fig. 4. This finding highlights the importance of the applied bias voltage and suggests that one has to be careful when interpreting the magnetic structure from experimentally observed SP-STM images. Based on this theoretical study, one can also conclude that the magnetic contrast reversal occurs between $0 \mathrm{~V}$ and $+1 \mathrm{~V}$ bias voltages. This contrast reversal is solely due to the sample electronic structure since the ideal magnetic tip is electronically featureless. Similarly, the sample electronic structure has been found responsible for a contrast reversal in Ref. [55].

Dependence of the magnetic contrast on the tip electronic structure can be studied by considering different tip models. As an example a ferromagnetic Ni tip has been chosen in Ref. [56]. Such tips are routinely used in SP-STM and STS experiments $[74,85]$. The Ni tip has been modeled by a seven-layer $\mathrm{Ni}$ film slab with (110) orientation, having one-one Ni apex atoms on both 


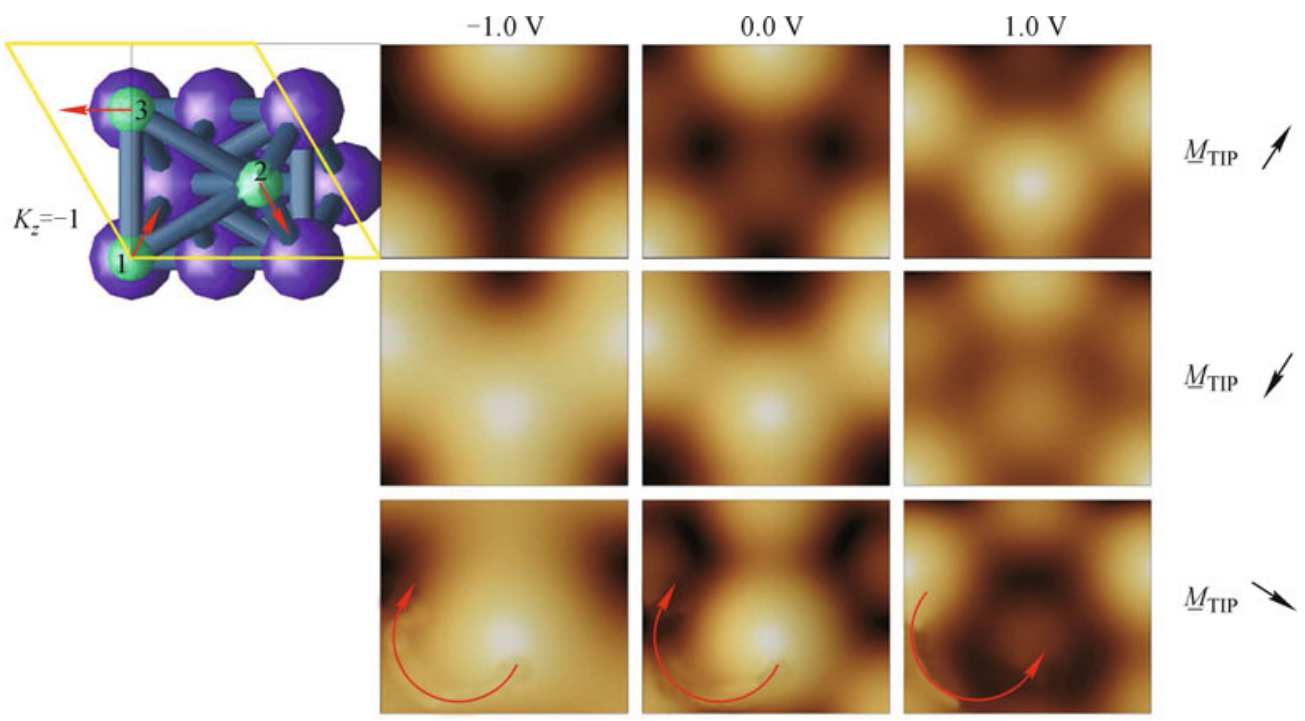

Fig. 4 Simulated SP-STM images depending on the bias voltage and the tip magnetization direction assuming an ideal electronically flat maximally spin-polarized tip. The magnetic contrast is reversed between $0.0 \mathrm{~V}$ and $1.0 \mathrm{~V}$. In the last row, the decreasing levels of Cr apparent heights are indicated by circular arrows. The surface geometry of $1 \mathrm{ML}$ Cr on $\operatorname{Ag}(111)$, its magnetic structure with $K_{z}=-1$ chirality, and the considered tip magnetization directions are explicitly shown, similarly as in Fig. 3. Reproduced from Ref. [56].

surfaces, i.e., with a double vacuum boundary. Here, the apex atom and the topmost surface layers have been relaxed on both sides. The interaction between apex atoms in neighboring supercells is minimized by choosing a $3 \times 3$ surface cell, and a $15.4 \AA$ wide separating vacuum region in $z$ direction. Moreover, an $11 \times 11 \times 1 \mathrm{MP} k$-point grid has been chosen for obtaining the projected DOS onto the apex atom. The electronic structure of the Ni apex is given in the top part of Figure 1 of Ref. [89]. A spin polarization of $P_{\mathrm{T}}=-0.91$ at the Fermi level, $E_{\mathrm{F}}^{\mathrm{T}}$, and
$\left|P_{\mathrm{T}}(E)\right|>0.8$ between $E_{\mathrm{F}}^{\mathrm{T}}-0.3 \mathrm{eV}$ and $E_{\mathrm{F}}^{\mathrm{T}}+0.3 \mathrm{eV}$ is obtained. Employing Eq. (17), the local electron work function above the tip apex is $\phi_{\mathrm{T}}=4.52 \mathrm{eV}$, and Eq. (15) has been used to determine the vacuum decay.

Figure 5 shows simulated SP-STM images for various tip magnetization directions at $-1 \mathrm{~V}, 0 \mathrm{~V}$ and $+1 \mathrm{~V}$ bias voltages including the electronic structure of the $\mathrm{Ni}$ tip into the tunneling model. By comparing images to those shown in Fig. 4 obtained by using an ideal magnetic tip, one finds that the magnetic contrast is the opposite for
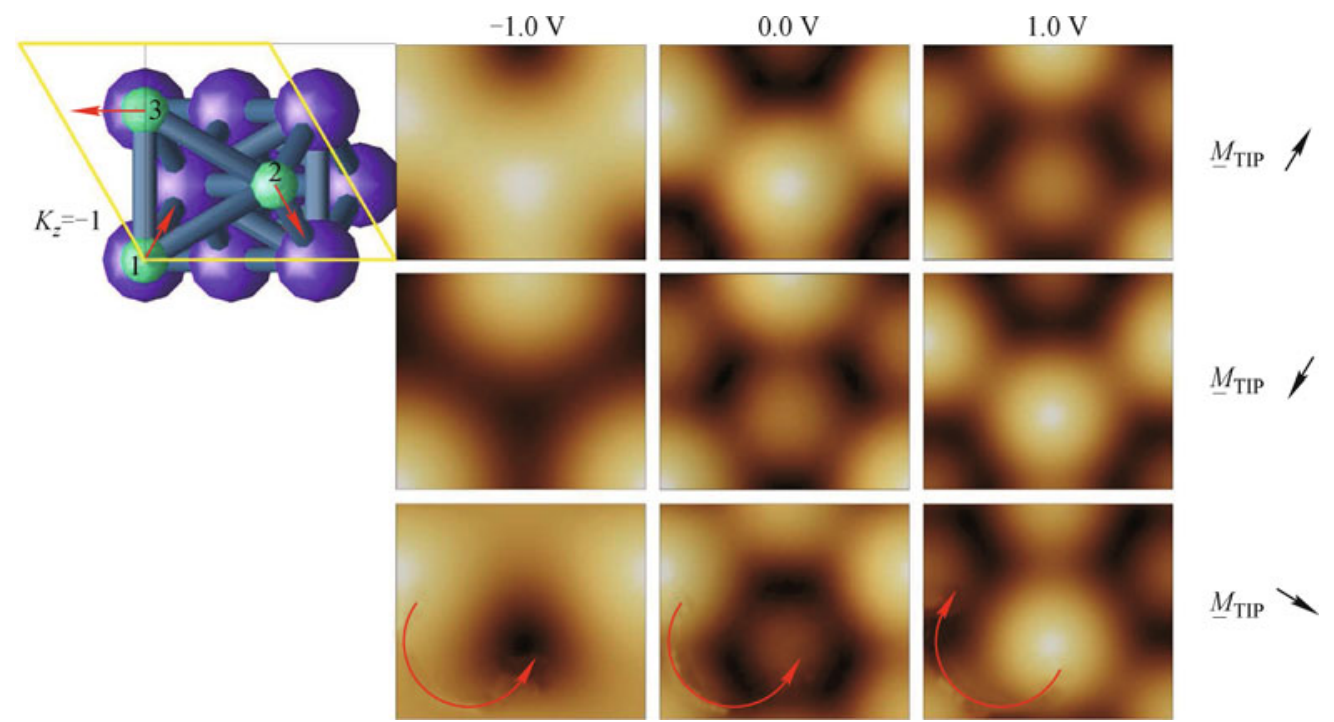

TIP

Fig. 5 Simulated SP-STM images depending on the bias voltage and the tip magnetization direction assuming a model $\mathrm{Ni}$ tip. The magnetic contrast is reversed compared to images obtained by using the ideal magnetic tip (compare to Fig. 4 ), and there is a bias dependent contrast reversal between $0.0 \mathrm{~V}$ and $1.0 \mathrm{~V}$. In the last row, the decreasing levels of Cr apparent heights are indicated by circular arrows. The surface geometry of 1 ML Cr on $\mathrm{Ag}(111)$, its magnetic structure with $K_{z}=-1$ chirality, and the considered tip magnetization directions are explicitly shown, similarly as in Fig. 3. Reproduced from Ref. [56]. 
each picture. This is due to the negative spin polarization $(-0.91)$ of the Ni tip apex at its Fermi level [89]. Note that the spin polarization of the ideal magnetic tip was assumed to be +1 in the whole energy range. Similarly as in Fig. 4, one finds qualitatively similar images for $-1 \mathrm{~V}$ and $0 \mathrm{~V}$ for the corresponding tip magnetization directions with higher magnetic contrast at $-1 \mathrm{~V}$ compared to $0 \mathrm{~V}$. Again, the magnetic contrast is reversed at $+1 \mathrm{~V}$ compared to the other two studied bias voltages. This effect is highlighted in the last row of Fig. 5 showing the decreasing levels of $\mathrm{Cr}$ apparent heights by circular arrows, thus, indicating a bias dependent apparent magnetic chirality. These results suggest that different tips can completely reverse the magnetic contrast. This effect has to be taken into account when determining the magnetic structure from experimentally observed SP-STM images.

\subsubsection{Tip material effect on the magnetic contrast}

Let us analyze the tip- and bias-voltage-dependent magnetic contrast in SP-STM images in more detail, e.g., between $\mathrm{Cr} 1$ and $\mathrm{Cr} 3$ atoms. In order to quantify the magnetic contrast between two surface sites at a given tip-sample distance, Eq. (69) or Eq. (72) has to be employed. For the determination of the vacuum decay constants $\kappa_{\mathrm{TOPO}}^{\mathrm{Cr} 1}(V)$ and $\kappa_{\mathrm{TOTAL}}^{a v . \mathrm{Cr} 1-\mathrm{Cr} 3}(V)$ in the denominator of these equations, the corresponding current values have to be calculated at least at two tip-sample distances. From expressing Eq. (70) at tip-sample distances $z_{1} \neq z_{2}$, the vacuum decay constant of the topographic current above atom $A$ can be given as

$$
\kappa_{\mathrm{TOPO}}^{A}(V)=\frac{\ln I_{\mathrm{TOPO}}^{A}\left(z_{1}, V\right)-\ln I_{\mathrm{TOPO}}^{A}\left(z_{2}, V\right)}{2\left(z_{2}-z_{1}\right)}
$$

Similarly, using Eq. (71), the corresponding vacuum decay constant can be obtained as

$$
\kappa_{\text {TOTAL }}^{a v . A B}(V)=\frac{\ln I_{\text {TOTAL }}^{a v \cdot A B}\left(z_{1}, V\right)-\ln I_{\text {TOTAL }}^{a v . A B}\left(z_{2}, V\right)}{2\left(z_{2}-z_{1}\right)}
$$

Note that though the absolute tip-sample distances $z_{1}$ and $z_{2}$ are unknown in experiments, the tip displacement $z_{2}-z_{1}$ can be experimentally obtained.

To prove that the exponentially decaying character of the corresponding currents with respect to the tip-sample distance $z$ in Eq. (70) and Eq. (71) is a valid assumption, a series of ordinary least squares linear regressions on the $\ln I(z, V)$ data were employed taking $150 z$ values in the range $[0.01 \AA, 7.95 \AA]$ for 500 bias voltages in the $[-2.5 \mathrm{~V}, 2.5 \mathrm{~V}]$ interval. For all considered bias voltages Pearson product-moment correlation coefficients better than $r(V)=-0.9999$ were obtained. These $r(V)$ values justify the exponentially decaying current assumption in Eq. (70) and Eq. (71) within the 3D WKB atom superposition approach. Note that orbital-dependent tunneling effects can modify this finding. The decay constants $\kappa_{\mathrm{TOPO}}^{\mathrm{Cr} 1}(V)$ and $\kappa_{\mathrm{TOT}}^{a v . \mathrm{Cr} 1-\mathrm{Cr} 3}(V)$ were determined from the linear regressions.

Figure 6 shows the bias dependent magnetic contrast between $\mathrm{Cr} 1$ and $\mathrm{Cr} 3$ atoms at $z=3.5 \AA$ tip-sample distance for both contrast formulas Eq. (69) and Eq. (72) using the ideal and Ni tip models. The tip magnetization direction is fixed parallel to the Cr1 magnetic moment. Due to the noncollinear magnetic structure, the $\mathrm{Cr} 2$ and Cr3 magnetic moments have an angle of $120^{\circ}$ with respect to the tip magnetization direction. Following this, the spin-polarized (MAGN) parts of the current measured above the $\mathrm{Cr} 2$ and $\mathrm{Cr} 3$ surface atoms are equal and their values are $\cos \left(120^{\circ}\right)=-0.5$ times that above Cr1, i.e.,

$$
I_{\mathrm{MAGN}}^{\mathrm{Cr} 2}=I_{\mathrm{MAGN}}^{\mathrm{Cr} 3}=-I_{\mathrm{MAGN}}^{\mathrm{Cr} 1} / 2
$$

The sign of the bias dependent contrast equals the sign of the spin-polarized part of the current above Cr1 [57], i.e.,

$$
\operatorname{sgn}\left(\Delta z^{\mathrm{Cr} 1-\mathrm{Cr} 3}\right)=\operatorname{sgn}\left(I_{\mathrm{MAGN}}^{\mathrm{Cr} 1}\right)
$$

This is understandable as the sign of $\Delta z^{\mathrm{Cr} 1-\mathrm{Cr} 3}$ is determined by the sign of its numerator $\Delta I_{\mathrm{MAGN}}^{\mathrm{Cr} 1-\mathrm{Cr} 3}$ since the denominator is always positive, and $\Delta I_{\mathrm{MAGN}}^{\mathrm{Cr} 1-\mathrm{Cr} 3}=$ $(3 / 2) I_{\mathrm{MAGN}}^{\mathrm{Cr} 1}$ because of Eq. (84). Accordingly, the contrast reversal is obtained at $\Delta z^{\mathrm{Cr} 1-\mathrm{Cr} 3}=0$, i.e., at

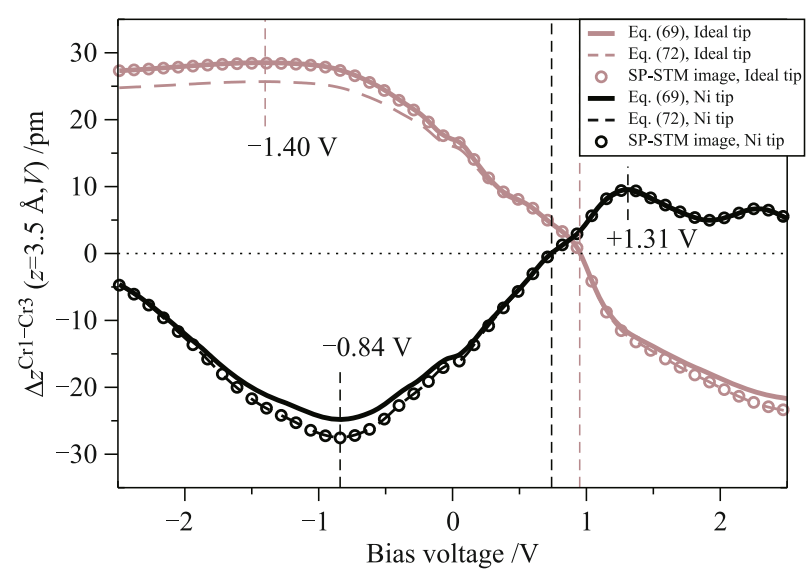

Fig. 6 The bias dependent magnetic contrast between $\mathrm{Cr} 1$ and $\mathrm{Cr} 3$ atoms $\Delta z^{\mathrm{Cr} 1-\mathrm{Cr} 3}(z=3.5 \AA, V)$ at $z=3.5 \AA$ tip-sample distance calculated using Eq. (69) (solid lines) and Eq. (72) (dashed lines) measured with the ideal magnetic tip (gray) and the Ni tip (black). The tip magnetization direction is fixed parallel to the Cr1 magnetic moment. The local absolute maxima of the magnetic contrasts and their bias values are explicitly shown in both the negative and the positive bias ranges. Vertical dashed lines at $0.74 \mathrm{~V}$ and $0.94 \mathrm{~V}$ denote the contrast reversals. For comparison, circle symbols show the apparent height difference between $\mathrm{Cr} 1$ and Cr3 atoms obtained from constant current SP-STM images, see text for details. Reproduced from Ref. [57]. 
$0.94 \mathrm{~V}$ and $0.74 \mathrm{~V}$ for the ideal and the Ni tip, respectively. From the calculated functions, bias voltages can be identified in both the negative and positive bias ranges, where a local absolute maximum magnetic contrast can be achieved. As can be seen, these highly depend on the spin polarization character of the magnetic tip. In the studied bias interval local absolute maximum contrasts are expected at $-1.40 \mathrm{~V}$ and $2.50 \mathrm{~V}$ for the measurement with the ideal tip, whereas these bias values are considerably modified to $-0.84 \mathrm{~V}$ and $1.31 \mathrm{~V}$ by using the $\mathrm{Ni}$ tip. The maximum absolute contrasts are obtained at the corresponding negative bias values for both tips. The reported bias positions do not depend on the employed magnetic contrast formula.

For validating the bias dependent magnetic contrast predictions, the apparent height difference between $\mathrm{Cr} 1$ and $\mathrm{Cr} 3$ atoms from constant current SP-STM images is calculated, that is also simulated within the $3 \mathrm{D} \mathrm{WKB}$ approach. The current contours are chosen in such a way that the apparent height of the $\mathrm{Cr} 1$ atom is $3.5 \AA$ at all considered bias voltages. The obtained data for the two considered tip models are shown in Fig. 6 using circle symbols. The qualitative agreement with the predicted magnetic contrasts using Eq. (69) and Eq. (72) is obvious at the first sight. Having a closer look one finds that Eq. (69) quantitatively reproduces the apparent height difference for $\Delta z^{\mathrm{Cr} 1-\mathrm{Cr} 3}>0$, and Eq. (72) for $\Delta z^{\mathrm{Cr} 1-\mathrm{Cr} 3}<0$. This means that both formulas are needed for a quantitative determination of the bias dependent magnetic contrast. In case one is interested in the identification of the bias voltage for obtaining the maximum contrast, the formula requiring less measurements or calculations, Eq. (69), can be applied.

In order to get more insight of the above finding that Eq. (69) quantitatively reproduces the apparent height difference for $\Delta z^{\mathrm{Cr} 1-\mathrm{Cr} 3}>0$, and Eq. (72) for $\Delta z^{\mathrm{Cr} 1-\mathrm{Cr} 3}<0$, a further analysis is needed. From Figure 6 it seems that the predicted magnetic contrast using Eq. (72) (dashed lines) is always smaller than using Eq.
(69) (solid lines). This means a larger absolute contrast for using Eq. (72) if the contrast is negative. Let us try to understand this difference. Therefore, a relation between the two contrast formulas $\Delta z_{I}^{\mathrm{Cr} 1-\mathrm{Cr} 3}$ in Eq. (69) and $\Delta z_{I I}^{\mathrm{Cr} 1-\mathrm{Cr} 3}$ in Eq. (72) is derived. Using Eq. (84) and that $I_{\mathrm{TOPO}}^{\mathrm{Cr} 3}=I_{\mathrm{TOPO}}^{\mathrm{Cr} 1}$, the average total current is

$$
\begin{aligned}
& I_{\mathrm{TOT}}^{a v \cdot \mathrm{Cr} 1-\mathrm{Cr} 3} \\
& \quad=\frac{\left(I_{\mathrm{TOPO}}^{\mathrm{Cr} 1}+I_{\mathrm{MAGN}}^{\mathrm{Cr} 1}\right)+\left(I_{\mathrm{TOPO}}^{\mathrm{Cr} 1}-I_{\mathrm{MAGN}}^{\mathrm{Cr} 1} / 2\right)}{2} \\
& \quad=I_{\mathrm{TOPO}}^{\mathrm{Cr} 1}+\frac{I_{\mathrm{MAGN}}^{\mathrm{Cr} 1}}{4}
\end{aligned}
$$

and thus,

$$
\begin{aligned}
\Delta z_{I I}^{\mathrm{Cr} 1-\mathrm{Cr} 3} & =\frac{\Delta I_{\mathrm{MAGN}}^{\mathrm{Cr} 1-\mathrm{Cr} 3}}{2 \kappa_{\mathrm{TOPO}}^{\mathrm{Cr} 1} I_{\mathrm{TOPO}}^{\mathrm{Cr} 1}+2 \kappa_{\mathrm{MAGN}}^{\mathrm{Cr} 1} I_{\mathrm{MAGN}}^{\mathrm{Cr} 1} / 4} \\
& =\frac{\Delta I_{\mathrm{MAGN}}^{\mathrm{Cr} 1-\mathrm{Cr} 3}}{2 \kappa_{\mathrm{TOPO}}^{\mathrm{Cr} 1} I_{\mathrm{TOPO}}^{\mathrm{Cr} 1}} \frac{1}{1+\frac{1}{4} \frac{\kappa_{\mathrm{MAGN}}^{\mathrm{Cr} 1} I_{\mathrm{MAGN}}^{\mathrm{Cr} 1}}{\kappa_{\mathrm{TOPO}}^{\mathrm{Cr} 1} I_{\mathrm{TOPO}}^{\mathrm{Cr} 1}}} \\
& =\frac{\Delta z_{I}^{\mathrm{Cr} 1-\mathrm{Cr} 3}}{1+\frac{1}{4} \frac{\kappa_{\mathrm{MAGN}}^{\mathrm{Cr} 1} I_{\mathrm{MAGN}}^{\mathrm{Cr} 1}}{\kappa_{\mathrm{TOPO}}^{\mathrm{Cr}} I_{\mathrm{TOPO}}^{\mathrm{Cr}}}}
\end{aligned}
$$

where the $(z, V)$ or $(V)$ arguments of the quantities are omitted for brevity, and an exponential decay for $I_{\mathrm{MAGN}}^{\mathrm{Cr} 1}(z, V)$ with respect to the tip-sample distance is assumed. Since the quantities $\kappa_{\mathrm{MAGN}}^{\mathrm{Cr} 1}, \kappa_{\mathrm{TOPO}}^{\mathrm{Cr} 1}$ and $I_{\mathrm{TOPO}}^{\mathrm{Cr} 1}$ are always positive, the sign of $I_{\mathrm{MAGN}}^{\mathrm{Cr} 1}$, i.e., the sign of the contrast [see Eq. (85)] determines the relation between $\Delta z_{I}^{\mathrm{Cr} 1-\mathrm{Cr} 3}$ and $\Delta z_{I I}^{\mathrm{Cr} 1-\mathrm{Cr} 3}$ : If $I_{\mathrm{MAGN}}^{\mathrm{Cr} 1}$ is positive then $0<\Delta z_{I I}^{\mathrm{Cr} 1-\mathrm{Cr} 3}<\Delta z_{I}^{\mathrm{Cr} 1-\mathrm{Cr} 3}$. On the other hand, if $I_{\mathrm{MAGN}}^{\mathrm{Cr} 1}<$ is negative then $\Delta z_{I I}^{\mathrm{Cr} 1-\mathrm{Cr} 3}<\Delta z_{I}^{\mathrm{Cr} 1-\mathrm{Cr} 3}<0$. This is exactly what can be observed in Fig. 6 .

In order to better visualize the bias dependent magnetic contrast, constant current SP-STM images have been simulated. Figure 7 shows such images measured with the ideal magnetic tip (top row) and the $\mathrm{Ni}$ tip (bottom row) at $0 \mathrm{~V}$, at the bias voltages corresponding to the contrast reversal $(0.94 \mathrm{~V}$ and $0.74 \mathrm{~V}$, respectively), and at the voltages corresponding to the local absolute
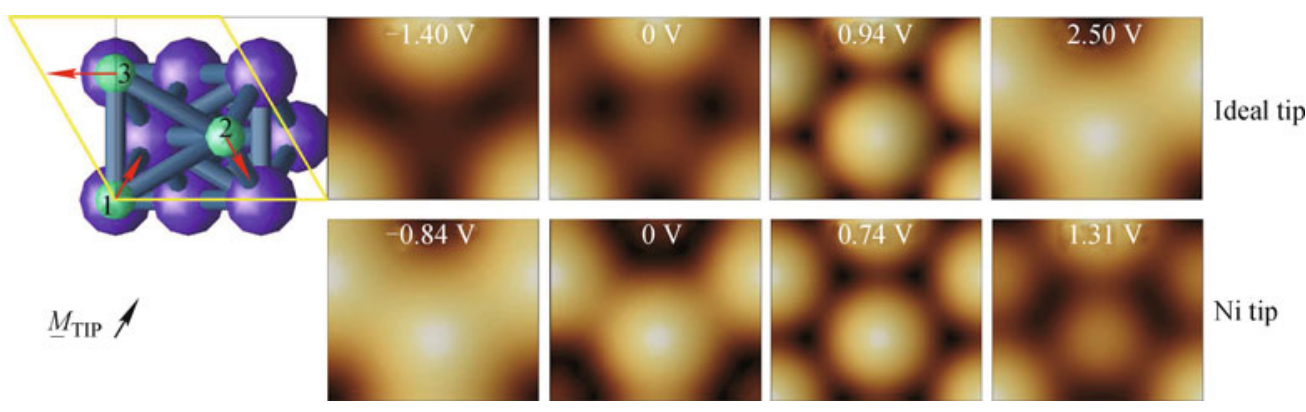

Fig. 7 Simulated SP-STM images depending on the bias voltage and the considered tip: ideal magnetic tip (top row), Ni tip (bottom row). The tip magnetization direction is fixed parallel to the Cr1 magnetic moment, and is indicated by a vector $\left(\underline{M}_{\mathrm{TIP}}\right)$. The bias values have been chosen corresponding to the local absolute maxima of the magnetic contrasts and the contrast reversals in Fig. 6. The surface geometry and the magnetic structure of $\mathrm{Cr} / \mathrm{Ag}(111)$ as well as the scanning area are also shown. Reproduced from Ref. [57]. 
maxima of the magnetic contrast in both the negative and positive ranges, i.e., at $-1.40 \mathrm{~V}$ and $2.50 \mathrm{~V}$ for the ideal tip, and at $-0.84 \mathrm{~V}$ and $1.31 \mathrm{~V}$ using the Ni tip. The surface geometry and the magnetic structure as well as the scanning area are also shown. The tip magnetization direction is fixed parallel to the Cr1 magnetic moment. For the zero bias images a temperature of $4.2 \mathrm{~K}$ was considered following Ref. [56] since there is no current at $0 \mathrm{~K}$.

A similar type of magnetic contrast at $0 \mathrm{~V}$ and at the negative bias voltages are found for both tips, respectively, i.e., for $V \leqslant 0, \Delta z^{\mathrm{Cr} 1-\mathrm{Cr} 3}>0(\mathrm{Cr} 1$ appears higher than $\mathrm{Cr} 2$ and $\mathrm{Cr} 3)$ for the ideal tip, and $\Delta z^{\mathrm{Cr} 1-\mathrm{Cr} 3}<0$ (Cr1 appears lower than $\mathrm{Cr} 2$ and $\mathrm{Cr} 3$ ) for the Ni tip, similarly as reported in Figs. 4-6. At the corresponding reversal voltages, $0.94 \mathrm{~V}$ (ideal tip) and $0.74 \mathrm{~V}(\mathrm{Ni}$ tip), all $\mathrm{Cr}$ atoms appear to be of equal height on the SP-STM image. Here, the magnetic contrast is lost. This corresponds to a qualitatively similar image of performing the STM measurement with a nonmagnetic tip, see $\underline{M}_{\mathrm{TIP}}=\underline{0}$ in Fig. 3. Above the reversal voltage, the magnetic contrast is inverted. This is illustrated by showing the images calculated at $2.50 \mathrm{~V}$ for the ideal tip, and at $1.31 \mathrm{~V}$ for the Ni tip in Fig. 7.

\subsubsection{Tip-sample distance effect on the magnetic contrast}

Let us analyze the effect of the tip-sample distance on the obtained results. Figure 8 shows the bias dependent magnetic contrast between $\mathrm{Cr} 1$ and $\mathrm{Cr} 3$ atoms calculated using Eq. (69) with the ideal magnetic tip at different tip-sample separations: $z=3.5,4.0,4.5$, and $5.0 \AA$. The tip magnetization direction is fixed parallel to the Cr1 magnetic moment. It is clearly seen that the bias positions of the contrast reversal and the local maxima are practically unaffected by the tip-sample distance. On the other hand, one finds that the absolute contrasts are decreasing with increasing tip-sample distance. Assuming an exponential decay, Eq. (73) has been put forward to determine the bias dependent magnetic contrast at arbitrary tip-sample distances from two contrast functions at fixed heights $z_{1} \neq z_{2}$. Taking the functions at $z_{1}=3.5$ and $z_{2}=4.5 \AA$, Eq. (73) is used to interpolate the bias dependent magnetic contrast to $z=4.0 \AA$, and to extrapolate to $z=5.0 \AA$. As the results obtained by the interpolation and the extrapolation agree quantitatively well with those calculated by using Eq. (69), the exponential decay of the absolute magnetic contrast with respect to the tip-sample distance and, thus, the validity of Eq. (73) are confirmed within the presented 3D WKB atom superposition framework. Note that orbitaldependent tunneling effects can modify this finding.

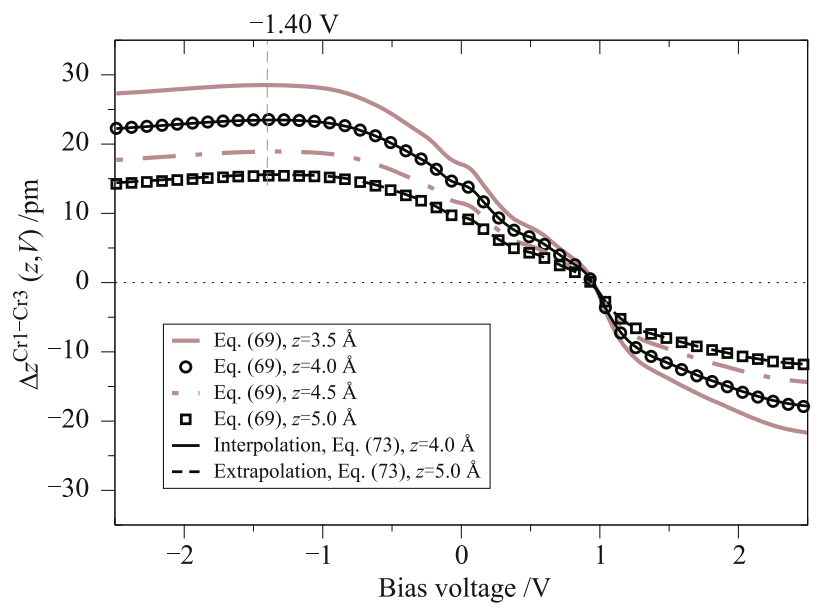

Fig. 8 The bias dependent magnetic contrast between $\mathrm{Cr} 1$ and Cr3 atoms $\Delta z^{\mathrm{Cr} 1-\mathrm{Cr} 3}(z, V)$ calculated using Eq. (69) with the ideal magnetic tip at different tip-sample separations: $z=3.5 \AA$ (gray solid line), $z=4.0 \AA$ (black circles), $z=4.5 \AA$ (gray dashdotted line), and $z=5.0 \AA$ (black squares). The tip magnetization direction is fixed parallel to the $\mathrm{Cr} 1$ magnetic moment. Taking the functions colored by gray at $z=3.5 \AA$ and $z=4.5 \AA$, Eq. (73) is used to interpolate the bias dependent magnetic contrast to $z=4.0$ $\AA$ (black solid line), and to extrapolate to $z=5.0 \AA$ (black dashed line). The absolute contrast maximum of each curve is found at $-1.40 \mathrm{~V}$, and is explicitly indicated. Reproduced from Ref. [57].

\subsubsection{Tip magnetization direction effect on the magnetic contrast}

Finally, let us analyze the effect of the magnetization orientation of the tip on the magnetic contrast. Figure 9 shows the bias dependent magnetic contrast between Cr1 and Cr3 atoms calculated by Eq. (69) at $z=3.5$ A tip-sample distance using the ideal magnetic tip at twelve in-plane magnetic directions rotated in steps of $30^{\circ}$. The unit vectors of these directions are explicitly shown for each curve. From the Figure it is clear that the bias positions of the contrast reversal and the local absolute maxima remain unaffected. The contrast curve indicated by the $(\mathrm{Cr} 1)$ direction is the same as the corresponding curves in Fig. 6 (ideal tip) and in Fig. 8 ( $z=3.5$ $\AA$ ). Moreover, one finds that the tip magnetization direction parallel or antiparallel to the $\mathrm{Cr} 2$ magnetic moment results in a bias-independent zero magnetic contrast between $\mathrm{Cr} 1$ and $\mathrm{Cr} 3$ atoms. This is clear since the magnetic currents above $\mathrm{Cr} 1$ and $\mathrm{Cr} 3$ atoms are equal in this case, similarly as the magnetic currents above $\mathrm{Cr} 2$ and $\mathrm{Cr} 3$ atoms are equal when the tip magnetization is parallel to the Cr1 moment, see Eq. (84). Apart from this, Fig. 9 shows that the magnetic contrast can be even more enhanced if the tip magnetization direction is changed from parallel to the $\mathrm{Cr} 1$ moment $(+0.500,+0.866)$ to the $(+0.866,+0.500)$ direction. This latter direction provides the largest achievable positive magnetic contrast between Cr 1 and Cr3 atoms at $-1.40 \mathrm{~V}$ bias. Turning the tip magnetization orientation to the opposite $(-0.866,-0.500)$ 
direction, a reversed curve is obtained. This direction of the tip magnetization enables to reach the largest negative magnetic contrast between $\mathrm{Cr} 1$ and $\mathrm{Cr} 3$ atoms at $-1.40 \mathrm{~V}$ bias. This finding suggests the possibility of tuning the magnetic contrast not only by changing the bias voltage but also by changing the tip magnetization direction.

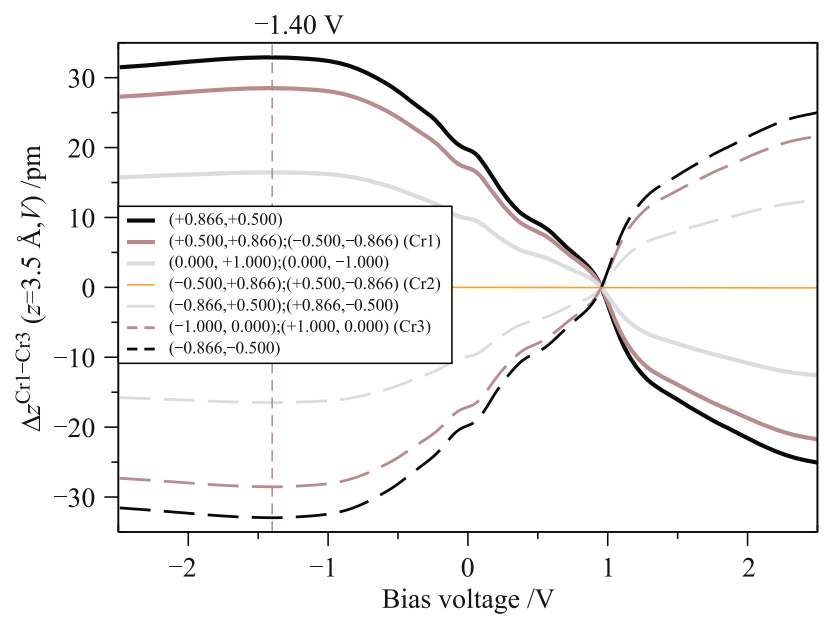

Fig. 9 The effect of the tip magnetization orientation on the bias dependent magnetic contrast between $\mathrm{Cr} 1$ and $\mathrm{Cr} 3$ atoms $\Delta z^{\mathrm{Cr} 1-\mathrm{Cr} 3}(z=3.5 \AA, V)$ at $z=3.5 \AA$ tip-sample distance calculated using Eq. (69) with the ideal magnetic tip. The unit vectors of the in-plane tip magnetization orientations are rotated in steps of $30^{\circ}$, and are explicitly shown. The parallel or antiparallel orientations with respect to the corresponding Cr magnetic moments are given in parentheses. The absolute contrast maximum of each curve is found at $-1.40 \mathrm{~V}$, and is explicitly indicated. Reproduced from Ref. [57].

\section{$3.5 \mathrm{~d} I / \mathrm{d} V$ contributions}

In the following, two distinctly different tip models are used than before: a magnetic $\mathrm{CrFe}$ tip, and an electronically flat magnetic tip based on the averaged electronic structure of the $\mathrm{CrFe}$ tip. The electronic structure data of the CrFe tip apex was taken from Ref. [76], where the tip was modeled as a single $\mathrm{Cr}$ apex atom on the $\mathrm{Fe}(001)$ surface. Ferriani et al. furthermore reported that an antiferromagnetic coupling of the $\mathrm{Cr}$ adatom to the $\mathrm{Fe}(001)$ surface is energetically preferred, and the vacuum spin polarization is fairly constant at around +0.8 in the energy range $\left[E_{\mathrm{F}}^{\mathrm{T}}-1 \mathrm{eV}, E_{\mathrm{F}}^{\mathrm{T}}+1 \mathrm{eV}\right][76]$. The local electron work function above the tip apex is assumed to be $\phi_{\mathrm{T}}=4.5 \mathrm{eV}$, that has been used to obtain the energy dependent vacuum decay in Eq. (15).

The electronically flat magnetic tip has been modeled based on the electronic structure of the $\mathrm{Cr}$ apex (PDOS) of the CrFe tip. The charge and absolute magnetization PDOS, $n_{\mathrm{T}}(E)$ and $\left|m_{\mathrm{T}}(E)\right|$, respectively, have been averaged in the $\left[E_{\mathrm{F}}^{\mathrm{T}}-2 \mathrm{eV}, E_{\mathrm{F}}^{\mathrm{T}}+2 \mathrm{eV}\right]$ range, and $n_{\mathrm{T}}=1.33 / \mathrm{eV}$ and $m_{\mathrm{T}}=1.06 / \mathrm{eV}$ were obtained. Thus, the spin polarization is $P_{\mathrm{T}}=m_{\mathrm{T}} / n_{\mathrm{T}}=+0.8$. In this case, the tip-derivative term of the differential conductance $D_{\mathrm{T}}(V)$ in Eq. (46) is zero, since $\partial n_{\mathrm{T}}(E) / \partial E=$ $\partial m_{\mathrm{T}}(E) / \partial E=0$. The electronic structure of the considered tip models and the surface $\mathrm{Cr}$ atom is given in Fig. 1 of Ref. [10]. The vacuum decay can be modeled using Eq. (15), where $\kappa(E, V)$ has an explicit $V$ dependence, and $\phi_{\mathrm{T}}=\phi_{\mathrm{S}}$ is assumed. Alternatively, a simpler model for $\kappa(E)$ can be considered without $V$ dependence as in Eq. (8). In this case the background term of the differential conductance $B(V)$ in Eq. (45) is zero, since the tunneling transmission does not depend on the bias voltage, and the physical differential conductance equals the virtual differential conductance, i.e., $\mathrm{d} I / \mathrm{d} V(V)=\mathrm{d} I / \mathrm{d} U(V, V)$. On the other hand, by assuming a $V$-dependent vacuum decay $\kappa(E, V), B(V)$ is not zero and it contributes to the total differential conductance, i.e., $\mathrm{d} I / \mathrm{d} V(V)=\mathrm{d} I / \mathrm{d} U(V, V)+B(V)$.

Figure 10 shows the bias dependence of the total tunneling current $I(V)$, calculated as the sum of Eqs. (37) and (38), at the position $z=3.5 \AA$ above a surface $\mathrm{Cr}$ atom probed with the $\mathrm{CrFe}$ tip having parallel (P) magnetization direction compared to the underlying surface $\mathrm{Cr}$ atom. Positive current means tunneling from the tip to the sample surface, whereas the current is negative in the opposite direction. One finds that the absolute value of the current is higher in the negative bias range compared to the positive range. This is due to the surface and tip electronic structures. The sample occupied PDOS combined with the tip unoccupied PDOS is greater than the sample unoccupied PDOS combined with the tip occupied PDOS [10]. Performing a numerical differentiation of $I(V)$ with respect to $V$, the differential conductance at this particular tip position is obtained.

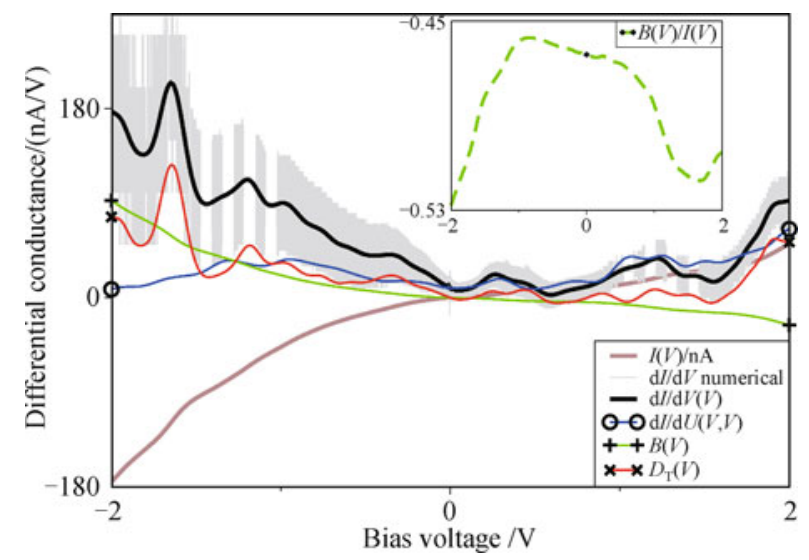

Fig. 10 Comparison of single point differential conductance tunneling spectra calculated from numerical differentiation of the tunneling current $I(V)$, and $\mathrm{d} I / \mathrm{d} V$ calculated according to Eq. (41), and its contributing terms, the virtual differential conductance $\mathrm{d} I / \mathrm{d} U(V, V)$, the background term $B(V)$, and the tip-derivative term $D_{\mathrm{T}}(V)$. The model CrFe tip apex is $3.5 \AA$ above a surface $\mathrm{Cr}$ atom and its magnetization direction is parallel to that of the underlying surface $\mathrm{Cr}$ atom. The inset shows the ratio of $B(V) / I(V)$. Reproduced from Ref. [10]. 
As can be seen this is extremely noisy, and a smoothing procedure should be applied to it before further analysis. Alternatively, the differential conductance can be calculated using Eq. (41), implemented within the 3D WKB atom superposition approach. Figure 10 shows that $\mathrm{d} I / \mathrm{d} V$ obtained this way (black curve) is a smooth function that fits precisely to the noisy numerical derivative of the current. There is more discussion about avoiding the numerical differentiation of the tunneling current in determining the $\mathrm{d} I / \mathrm{d} V$, e.g., in Ref. [16].

More information about the $\mathrm{d} I / \mathrm{d} V$ can be obtained by analyzing its components, the virtual differential conductance $\mathrm{d} I / \mathrm{d} U(V, V)$, the background term $B(V)$, and the tip-derivative term $D_{T}(V)$. One finds that $\mathrm{d} I / \mathrm{d} U(V, V)$ differs less than $10 \%$ compared to $\mathrm{d} I / \mathrm{d} V$ in the bias range $[-0.01 \mathrm{~V},+0.01 \mathrm{~V}]$ only, i.e., practically at the common Fermi level of the tip and the sample. Outside the given bias range, the relative difference between $\mathrm{d} I / \mathrm{d} U(V, V)$ and $\mathrm{d} I / \mathrm{d} V$ is more than $10 \%$. This means that the virtual differential conductance approximation for the $\mathrm{d} I / \mathrm{d} V$ (also known as the LDOS approximation) is not sufficient for nonzero bias. Note that at zero bias the two quantities are identical, $\mathrm{d} I / \mathrm{d} V(0)=$ $\mathrm{d} I / \mathrm{d} U(0,0)$. Moreover, one can recognize that most part of the $\mathrm{d} I / \mathrm{d} V$ peak structure is already included in the $\mathrm{d} I / \mathrm{d} U(V, V)$ term, which is qualitatively similar to the charge PDOS of the surface $\mathrm{Cr}$ atom of the sample, $n_{\mathrm{S}}(E)$ [10]. Apart from this, the peak structure of $D_{\mathrm{T}}(V)$, calculated via Eqs. (50)-(51), clearly shows up in the $\mathrm{d} I / \mathrm{d} V$, particularly pronounced at high bias voltages. The reason is the rapidly changing tip electronic structure in these energy regions [10]. The features from $\mathrm{d} I / \mathrm{d} U(V, V)$ and $D_{\mathrm{T}}(V)$ are transferred to the physical differential conductance, since $B(V)$, calculated via Eqs. (48) and (49), is smooth compared to the other two components in the whole bias range. Moreover, one finds that $B(V)$ is a monotonic function of the bias voltage, and it is nearly proportional to $I(V)$ as has been reported earlier for different levels of STS theories [7, 87]. The proportionality function $B(V) / I(V)$ is plotted in the inset of Fig. 10. It can be seen that its sign is in agreement with Ref. [7] and it has a non-trivial bias dependence. This is essentially due to the extra $1 / \kappa(E, V)$ factor in the energy integration of the background term, Eqs. (48) and (49), compared to the tunneling current expression. More discussion on $B(V) / I(V)$ can be found in Ref. [10]. In the following the focus will be on the comparison of SP-STS spectra by reversing the tip magnetization direction, and also using the flat magnetic tip model.

\subsection{Tip effects on the SP-STS spectra}

Figure 11 shows simulated single point differential con- ductance tunneling spectra following Eq. (41), probed with the flat magnetic tip and the model CrFe tip, $z=3.5 \AA$ above a surface Cr atom. Parallel (P) and antiparallel (AP) tip magnetization directions are set relative to the underneath surface $\mathrm{Cr}$ atom. It can clearly be seen that measuring the spectra with oppositely magnetized tips of the same type result in different differential conductance curves, in agreement with SP-STS experiments performed on oppositely magnetized sample areas with a fixed tip magnetization direction [73, 75]. For the flat magnetic tip, two different vacuum decays, $\kappa(E)$ and $\kappa(E, V)$ are assumed using Eqs. (8) and (15), respectively. For the bias-independent vacuum decay (dotted curves) one finds that $\mathrm{d} I^{\mathrm{P}} / \mathrm{d} V>\mathrm{d} I^{\mathrm{AP}} / \mathrm{d} V$ below $V=+0.54 \mathrm{~V}$, while $\mathrm{d} I^{\mathrm{P}} / \mathrm{d} V<\mathrm{d} I^{\mathrm{AP}} / \mathrm{d} V$ above $V=+0.54 \mathrm{~V}$. In Ref. [89] the effective spin polarization $\left[\boldsymbol{P}_{\mathrm{T}}(E) \boldsymbol{P}_{\mathrm{S}}(E)=\boldsymbol{m}_{\mathrm{T}}(E) \boldsymbol{m}_{\mathrm{S}}(E) /\left(n_{\mathrm{T}}(E) n_{\mathrm{S}}(E)\right)\right]$ has been identified responsible for such an effect. This is the decisive factor for determining the sign of the magnetic contribution to $\mathrm{d} I / \mathrm{d} V$ at energy $E$ in the SP-STS model presented in Section 2.2 as well. The magnetic part of the physical differential conductance is given in Eq. (43). Since the vacuum decay does not depend on the bias voltage $V$ for the dotted curves, and the tip is electronically flat, $\mathrm{d} I_{\mathrm{MAGN}} / \mathrm{d} V(V)=\mathrm{d} I_{\mathrm{MAGN}} / \mathrm{d} U(V, V)$. Thus, the sign change of $\mathrm{d} I_{\mathrm{MAGN}} / \mathrm{d} V$ occurs at the sign change of $\mathrm{d} I_{\text {MAGN }} / \mathrm{d} U(V, V)$, i.e., at the reversal of the sample spin polarization vector at $0.54 \mathrm{eV}$ above the sample Fermi level [56]. For the flat magnetic tip and the

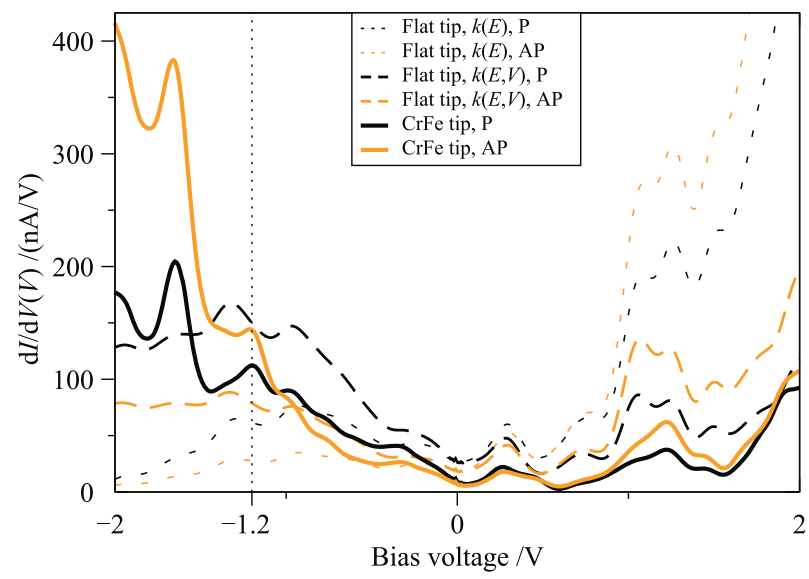

Fig. 11 Comparison of simulated single point differential conductance tunneling spectra following Eq. (41), probed with the flat magnetic tip and the model CrFe tip, $3.5 \AA$ above a surface $\mathrm{Cr}$ atom. Parallel (P) and antiparallel (AP) tip magnetization directions are set relative to the underneath surface $\mathrm{Cr}$ atom. For the flat magnetic tip, two different vacuum decays, $\kappa(E)$ and $\kappa(E, V)$ are assumed using Eqs. (8) and (15), respectively. The vertical dotted line at $-1.2 \mathrm{~V}$ shows the bias position of the identified STS peak coming from the electronic structure of the CrFe tip. Reproduced from Ref. [10]. 
assumed bias dependent vacuum decay (dashed curves) one finds that $\mathrm{d} I^{\mathrm{P}} / \mathrm{d} V>\mathrm{d} I^{\mathrm{AP}} / \mathrm{d} V$ below $V=+0.5$ $\mathrm{V}$, and $\mathrm{d} I^{\mathrm{P}} / \mathrm{d} V<\mathrm{d} I^{\mathrm{AP}} / \mathrm{d} V$ above $V=+0.5 \mathrm{~V}$, i.e., the sign change of the magnetic component is slightly shifted toward zero bias. The reason is the nonzero background term $B_{\text {MAGN }}(V)$ due to $\kappa(E, V)$, and $\mathrm{d} I_{\text {MAGN }} / \mathrm{d} V(V)=$ $\mathrm{d} I_{\text {MAGN }} / \mathrm{d} U(V, V)+B_{\text {MAGN }}(V)$ has to be considered. Note that $D_{\mathrm{T}}^{\mathrm{MAGN}}(V)$ is still zero because of the constant tip magnetization PDOS. Comparing the two vacuum decay models for the flat tip, it is clear that the topographic part of the background term has another effect on the heights of the spectra, i.e., they are enhanced and reduced in the negative and positive bias ranges, respectively, compared to the $\kappa(E)$ model. On the other hand, the features of the spectra (peaks and dips) occur at the same bias positions for both vacuum decay models.

The inclusion of a realistic tip electronic structure into the SP-STS model complicates the spectra even more. This is demonstrated in Fig. 11 for the CrFe tip model (solid lines). In this case all three terms contribute to the differential conductance, and $\mathrm{d} I / \mathrm{d} V(V)=$ $\mathrm{d} I / \mathrm{d} U(V, V)+B(V)+D_{\mathrm{T}}(V)$. Thus, the relative heights of the differential conductance tunneling spectra $\mathrm{d} I^{\mathrm{P}} / \mathrm{d} V$ and $\mathrm{d} I^{\mathrm{AP}} / \mathrm{d} V$ are determined by the superposition of the magnetic $\mathrm{d} I_{\mathrm{MAGN}} / \mathrm{d} U(V, V), B_{\mathrm{MAGN}}(V)$, and $D_{\mathrm{T}}^{\mathrm{MAGN}}(V)$ terms. The role of the effective spin polarization is more complicated, since, apart from the $\mathrm{d} I_{\text {MAGN }} / \mathrm{d} U(V, V)$ term, it appears in the $\mathrm{d} I / \mathrm{d} V$ expression through the bias-integrated quantities $B_{\text {MAGN }}(V)$ and $D_{\mathrm{T}}^{\mathrm{MAGN}}(V)$. For the $\mathrm{P}$ tip magnetization, $\mathrm{d} I^{\mathrm{P}} / \mathrm{d} V$ is the same as the black solid curve in Fig. 10, and its contributions are also shown there. In Fig. 11, more changes of the relative height of the $\mathrm{d} I^{\mathrm{P}} / \mathrm{d} V$ and $\mathrm{d} I^{\mathrm{AP}} / \mathrm{d} V$ spectra measured with the CrFe tip than with the flat tip are observed. These include the sign changes of the magnetic part of the spectra, similarly as before. One finds that $\mathrm{d} I^{\mathrm{P}} / \mathrm{d} V>\mathrm{d} I^{\mathrm{AP}} / \mathrm{d} V$ in the bias interval $[-1.04 \mathrm{~V}$, $+0.49 \mathrm{~V}]$, and a reversed relation is obtained in the complementary bias regime. Comparing the spectra to the ones calculated with the flat magnetic tip, one can see that they are qualitatively closer to the $\kappa(E, V)$ model used for the flat tip due to the presence of the background terms. Moreover, the individual features coming from the sample and the tip electronic structures can be assigned. In the presented case the peak at $-1.2 \mathrm{~V}$, indicated by a vertical dotted line in Figure 11, is identified coming from the $\mathrm{CrFe}$ tip electronic structure since it is missing from the spectra calculated with the flat tip. All other features are related to the sample electronic structure as they appear in the spectra measured with the flat tip.

\subsection{Tip effects on the magnetic asymmetry}

The relative heights of the differential conductance tunneling spectra $\mathrm{d} I^{\mathrm{P}} / \mathrm{d} V$ and $\mathrm{d} I^{\mathrm{AP}} / \mathrm{d} V$ can also be determined from the magnetic asymmetry, Eq. (74). Let us compare the magnetic asymmetries calculated from the spectra in Fig. 11 using the two magnetic tips. Moreover, for the CrFe tip, the asymmetry expressions defined in Eqs. (76)-(80) are compared to estimate the error one makes when neglecting the background and tip-related components of $\mathrm{d} I / \mathrm{d} V(V)$. Figure 12 shows the calculated asymmetry functions at $z=3.5 \AA$ above a surface $\mathrm{Cr}$ atom. It can be seen that $A^{\text {Flat, } \kappa(E)}(V)$ and $A^{\text {Flat }, \kappa(E, V)}(V)$ (dashed curves) behave qualitatively similarly. In addition, $A^{\text {Flat, } \kappa(E)}(V)$ is greater than $A^{\text {Flat }, \kappa(E, V)}(V)$ in almost the full studied bias range. The opposite relation holds between $0 \mathrm{~V}$ and $+0.3 \mathrm{~V}$ only, however, the relative difference between the two quantities is less than $1.4 \%$ in this regime. Moreover, these two magnetic asymmetries are within $5 \%$ relative difference in the bias range $[-0.23 \mathrm{~V},+0.31 \mathrm{~V}]$.

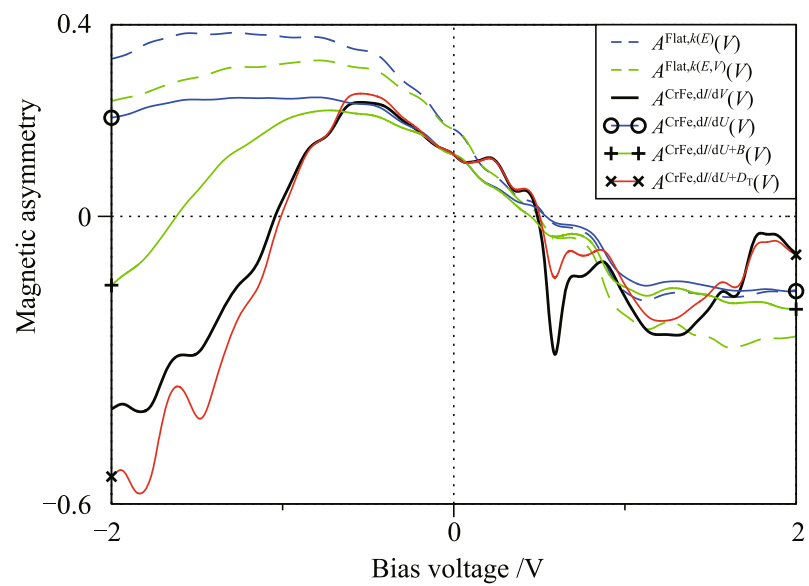

Fig. 12 Comparison of magnetic asymmetries $3.5 \AA$ above a surface $\mathrm{Cr}$ atom probed with the flat magnetic tip and the model $\mathrm{CrFe}$ tip. $A^{\text {Flat }, \kappa(E)}, A^{\text {Flat }, \kappa(E, V)}$, and $A^{\mathrm{CrFe}, \mathrm{d} I / \mathrm{d} V}$ are calculated from the corresponding $\mathrm{P}$ and $\mathrm{AP}$ spectra shown in Fig. 11. For the $\mathrm{CrFe}$ tip we compare the magnetic asymmetry expressions defined in Eqs. (76)-(80). Reproduced from Ref. [10].

Considering the $\mathrm{CrFe}$ tip, the experimentally measurable magnetic asymmetry $A^{\mathrm{CrFe}, \mathrm{d} I / \mathrm{d} V}(V)$ (black solid curve) is qualitatively different from the two asymmetry functions calculated with the flat tip, e.g., it has a richer structure at positive bias voltages. More importantly, it has an extra sign change occurring at -1.04 $\mathrm{V}$ apart from $+0.49 \mathrm{~V}$. These correspond to the height changes of $\mathrm{d} I^{\mathrm{P}} / \mathrm{d} V$ and $\mathrm{d} I^{\mathrm{AP}} / \mathrm{d} V$ relative to each other in Fig. 11. Let us estimate the error of the magnetic asymmetry when neglecting the background and the tipderivative terms. According to Eq. (77), $A^{\mathrm{CrFe}, \mathrm{d} I / \mathrm{d} U}(V)$ (curve with symbol "o") considers the virtual differen- 
tial conductances only. It is within $10 \%$ relative error compared to $A^{\mathrm{CrFe}, \mathrm{d} I / \mathrm{d} V}(V)$ in the bias range $[-0.65$ $\mathrm{V},+0.1 \mathrm{~V}]$. However, its sign does not correspond to $A^{\mathrm{CrFe}, \mathrm{d} I / \mathrm{d} V}(V)$ in the bias intervals $[-2 \mathrm{~V},-1.04 \mathrm{~V}]$ and $[+0.49 \mathrm{~V},+0.54 \mathrm{~V}]$. Adding the background term $B(V)$ to $\mathrm{d} I / \mathrm{d} U(V, V)$ results in an improved differential conductance expression, and $A^{\mathrm{CrFe}, \mathrm{d} I / \mathrm{d} U+B}(V)$ (curve with symbol "+"), defined in Eq. (79), behaves qualitatively similarly to $A^{\mathrm{CrFe}, \mathrm{d} I / \mathrm{d} U}(V)$ above $-0.65 \mathrm{~V}$. However, its sign change is shifted to $+0.45 \mathrm{~V}$ from $+0.54 \mathrm{~V}$. Additionally, a sign change in the negative bias range occurs at $-1.62 \mathrm{~V}$. Close to the sample Fermi level, $A^{\mathrm{CrFe}, \mathrm{d} I / \mathrm{d} U+B}(V)$ is within $10 \%$ relative error compared to $A^{\mathrm{CrFe}, \mathrm{d} I / \mathrm{d} V}(V)$ in a decreased bias range of $[-0.34$ $\mathrm{V},+0.1 \mathrm{~V}]$. Finally, by adding the tip-derivative term $D_{\mathrm{T}}(V)$ to $\mathrm{d} I / \mathrm{d} U(V, V), A^{\mathrm{CrFe}, \mathrm{d} I / \mathrm{d} U+D_{\mathrm{T}}}(V)$ (curve with symbol "x"), defined in Eq. (80), shows the most closely related shape to $A^{\mathrm{CrFe}, \mathrm{d} I / \mathrm{d} V}(V)$. Furthermore, it is also quantitatively close to the physical magnetic asymmetry as its sign changes occur at $-1.01 \mathrm{~V}$ and $+0.5 \mathrm{~V}$, and it is within $10 \%$ relative error compared to $A^{\mathrm{CrFe}, \mathrm{d} I / \mathrm{d} V}(V)$ in an increased bias interval $[-0.90 \mathrm{~V},+0.45 \mathrm{~V}]$. Summarizing this paragraph, the contribution of all three terms to the $\mathrm{d} I / \mathrm{d} V(V)$ according to Eq. (41) is needed to define the physical magnetic asymmetry, that can meaningfully be compared to experiments.

\subsection{Two-dimensional local spectroscopic information}

The methods presented in Sections 2.4.3 and 2.4.4 also enable one to simulate two-dimensional (2D) $\mathrm{d} I / \mathrm{d} V$ and effective spin polarization (ESP) maps in high spatial resolution above the surface, that can be compared to results of SP-STS experiments. Such experiments are routinely performed while the tip follows a constant TOTAL current contour, see, e.g., Ref. [111]. Figure 13 illustrates this capability of the 3D WKB method, where the flat tip model with tip magnetization direction $\underline{M}_{\text {TIP }}$ parallel to the Cr1 magnetic moment has been used. Moreover, $\kappa(E, V)$, Eq. (15) has been used for the vacuum decay. By choosing $V_{\text {stab }}=+1 \mathrm{~V}$, the 3D TOTAL current map in a finite box above the surface is calculated. From this $3 \mathrm{D}$ data, the current contour of $I_{\mathrm{TOTAL}}=54$ $\mathrm{nA}$ is extracted, which is around $3.5 \AA$ above the sample surface and has a corrugation of $4.2 \mathrm{pm}$. This contour, $z\left(x, y, V_{\text {stab }}=+1 \mathrm{~V}, I_{\text {TOTAL }}=54 \mathrm{nA}\right)$ is plotted in the bottom left part of Fig. 13. For calculating the differential conductance-related 2D maps, the vertical position $z$ of the tip apex atom is varied following this constant current contour. Thus, spin-resolved $\mathrm{d} I / \mathrm{d} V$ and magnetic asymmetry maps can be simulated at different $V$ bias voltages corresponding to experiments. As an example, $\mathrm{d} I / \mathrm{d} V(x, y)$ and $\operatorname{ESP}(x, y)$, see Eq. (78), are shown in the middle and right columns of Fig. 13, respectively, calculated at bias voltages $V=+0.5 \mathrm{~V}$ (top) and $V=+0.6$ $\mathrm{V}$ (bottom). These voltages have been chosen close to the spin polarization reversal of the sample surface at 0.54 $\mathrm{eV}$ above its Fermi level, see Ref. [56]. Indeed, the reversal of the $2 \mathrm{D} \mathrm{d} I / \mathrm{d} V$ map at $V=+0.6 \mathrm{~V}$ compared to $V=+0.5 \mathrm{~V}$ can clearly be seen. While the SP-STM image at $+1 \mathrm{~V}$ and the $\mathrm{d} I / \mathrm{d} V$ map at $+0.6 \mathrm{~V}$ show the same type of contrast, the $\mathrm{d} I / \mathrm{d} V$ signal is inverted for $+0.5 \mathrm{~V}$. Since $P_{\mathrm{T}}=+0.8$ is constant in the full energy range, this effect is due to the surface electronic structure. At $+0.6 \mathrm{~V}$ bias, all surface $\mathrm{Cr}$ spin polarization vectors point opposite to their local magnetic moment directions [56], and since $P_{\mathrm{T}}=+0.8$ is set with respect to the $(1 / 2, \sqrt{3} / 2)$ direction $\left(\underline{M}_{\mathrm{TIP}}\right)$, the leading term of the magnetic differential conductance, $\mathrm{d} I_{\mathrm{MAGN}} / \mathrm{d} U(V, V)$ is negative above the surface $\mathrm{Cr} 1$ atom. Moreover, the sign of $\mathrm{d} I_{\text {MAGN }} / \mathrm{d} U(V, V)$ changes to positive above the other two $\mathrm{Cr}$ atoms in the magnetic unit cell. This results in the minimal total $\mathrm{d} I / \mathrm{d} V(x, y)$ above the $\mathrm{Cr} 1$ atom $(22.9$ $\mathrm{nA} / \mathrm{V})$, whereas above the other two $\mathrm{Cr}$ atoms $\mathrm{d} I / \mathrm{d} V$ is maximal $(23.6 \mathrm{nA} / \mathrm{V})$. This happens even though the topographic differential conductance is higher above the $\mathrm{Cr} 1$ atom, which is lower-lying on the constant current contour. Similarly, the case of $+0.5 \mathrm{~V}$ is reversed, since all surface $\mathrm{Cr}$ spin polarization vectors point along their local magnetic moment directions [56] and the maximal total $\mathrm{d} I / \mathrm{d} V(x, y)$ is achieved above the $\mathrm{Cr} 1$ atom $(16.5 \mathrm{nA} / \mathrm{V})$, whereas above the other two $\mathrm{Cr}$ atoms $\mathrm{d} I / \mathrm{d} V$ is lower $(16.0 \mathrm{nA} / \mathrm{V})$. The minimal $\mathrm{d} I / \mathrm{d} V=15.8$ $\mathrm{nA} / \mathrm{V}$ is obtained above the midpoint of the lines connecting two $\mathrm{d} I / \mathrm{d} V$ maxima. If we introduce the notation of $\mathrm{d} I^{\mathrm{P}} / \mathrm{d} V(x, y)$ for the above calculated differential conductances with P parallel to the indicated $\underline{M}_{\mathrm{TIP}}$ direction in Fig. 13, then the antiparallel tip orientation is denoted by $\mathrm{AP}$, and $\mathrm{d} I^{\mathrm{AP}} / \mathrm{d} V(x, y)$ can similarly be calculated. For the very same reason as discussed, a reversed tip magnetization direction would result in a reversed $\mathrm{d} I^{\mathrm{AP}} / \mathrm{d} V$ map concerning the heights above the non-equivalent magnetic $\mathrm{Cr}$ atoms. Thus, at $+0.6 \mathrm{~V}$ the difference between $\mathrm{d} I^{\mathrm{P}} / \mathrm{d} V(x, y)$ and $\mathrm{d} I^{\mathrm{AP}} / \mathrm{d} V(x, y)$ is minimal and negative above the $\mathrm{Cr} 1$ atom, and maximal and positive above the other two $\mathrm{Cr}$ atoms, while the opposite is true at $+0.5 \mathrm{~V}$. These explain qualitatively well the simulated $\operatorname{ESP}(x, y)$ maps, see the right column of Fig. 13. The $\operatorname{ESP}(x, y)=0$ contour acts as a border between surface regions with positive and negative ESP at the given bias. Note that the sign of the tip spin polarization has a crucial effect on the $\operatorname{ESP}(x, y)$ map. Reversing the sign of $P_{T}$ compared to the $\underline{M}_{\mathrm{TIP}}$ direction would result in a reversed $\operatorname{ESP}(x, y)$ map.

Applying the presented 3D WKB method to magnetic 

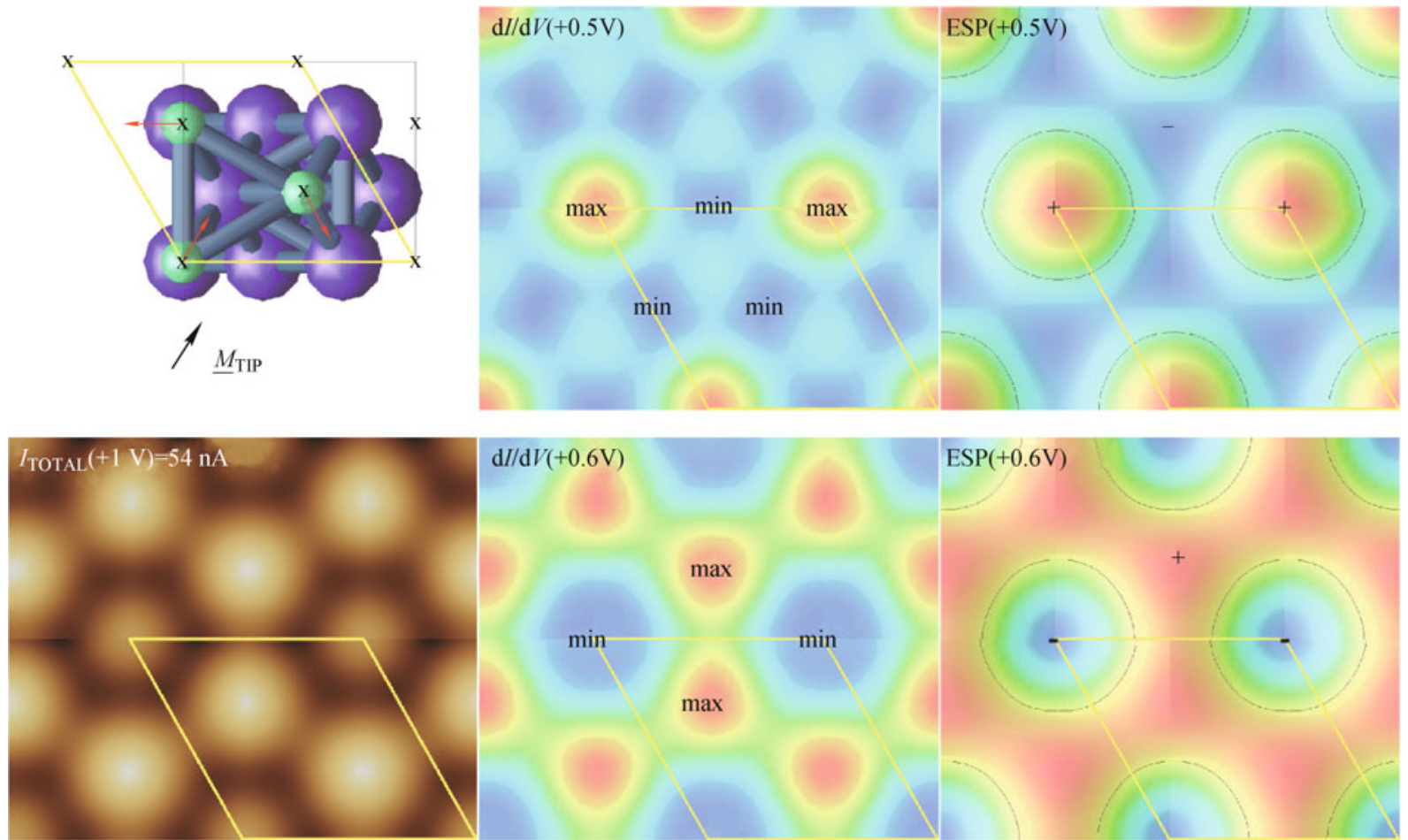

Fig. 13 Top left: Surface geometry of $1 \mathrm{ML} \mathrm{Cr}$ on $\mathrm{Ag}(111)$ and the magnetic ground state with $K_{z}=-1$ chirality. For explanation, see the caption of Fig. 3. The $(\sqrt{3} \times \sqrt{3})$ magnetic unit cell is drawn by yellow (light gray) color. The surface $\mathrm{Cr}$ positions are denoted by "x". The tip magnetization direction $\left(\underline{M}_{\mathrm{TIP}}\right)$ is indicated by an arrow. Bottom left: Constant current contour at about $3.5 \AA$ above the surface with $I_{\text {TOTAL }}\left(V_{\text {stab }}=+1 \mathrm{~V}\right)=54 \mathrm{nA}$ calculated with the flat magnetic tip using $\kappa(E, V)$, Eq. (15). Middle column: Simulated 2D differential conductance maps $\mathrm{d} I / \mathrm{d} V(x, y, V=+0.5 \mathrm{~V})($ top middle; min. 15.8, max. $16.5 \mathrm{nA} / \mathrm{V})$, and $\mathrm{d} I / \mathrm{d} V(x, y, V=+0.6 \mathrm{~V})$ (bottom middle; min. 22.9, max. $23.6 \mathrm{nA} / \mathrm{V})$, while the tip is following the constant current contour at the bottom left part of the Figure. Minimum (MIN) and maximum (MAX) values are indicated. Right column: Simulated effective spin polarization (ESP) maps on the same current contour following Eq. (78), $\operatorname{ESP}(x, y, V=+0.5 \mathrm{~V})$ (top right), and $\operatorname{ESP}(x, y, V=+0.6 \mathrm{~V})$ (bottom right). Black contours correspond to zero ESP, and the regions with positive $(+)$ and negative (-) ESP are indicated. The surface magnetic unit cell is drawn by a yellow (light gray) rhombus on each 2D map. Reproduced from Ref. [10].

surfaces, two-dimensional $\mathrm{d} I^{\mathrm{P}} / \mathrm{d} V(x, y), \mathrm{d} I^{\mathrm{AP}} / \mathrm{d} V(x, y)$, and magnetic asymmetry $A(x, y)$ maps can be constructed on appropriate current contours at arbitrary $V$ bias, corresponding to SP-STS experiments. Similarly, an $\operatorname{ESP}(x, y)$ map can be simulated. It has to be stressed again that the ESP can not simply be obtained from experimental magnetic asymmetry due to the presence of the background and tip-derivative terms. By explicitly considering the tip electronic structure in the SPSTS model based on experimental information, it could help in a more reasonable interpretation of experimentally measured tunneling spectra, magnetic asymmetries, and effective spin polarization.

\section{Corrugation inversion due to orbital effects: $\mathrm{W}(\mathbf{1 1 0})$}

In order to demonstrate the capabilities of the orbital dependent tunneling model presented in Section 2.3, a $\mathrm{W}(110)$ surface is considered. This surface has technolog- ical importance as it is widely used as substrate for thin film growth, see e.g., Refs. [44, 112]. As it was pointed out by Heinze et al. [112], the determination of the position of surface atomic sites is not straightforward as atomic resolution is lost at negative bias voltages, and a biasdependent corrugation reversal has been predicted. This means that normal and anticorrugated constant current STM images can be obtained in certain bias voltage ranges, and the $\mathrm{W}$ atoms do not always appear as protrusions in the images. It was shown that a competition between states from different parts of the surface Brillouin zone is responsible for this effect $[112,113]$. Here, the corrugation reversal effect is reviewed based on Ref. [91].

\subsection{Computational details}

Geometry relaxation and electronic structure calculations were performed based on the Density Functional Theory (DFT) within the Generalized Gradient Approximation (GGA) implemented in the Vienna Ab-initio 
Simulation Package (VASP) [103-105]. A plane wave basis set for electronic wave function expansion together with the projector augmented wave (PAW) method [106] has been applied, and the exchange-correlation functional is parametrized according to Perdew and Wang (PW91) [107]. The electronic structures of the sample surface and the tip have been calculated separately.

The W(110) surface is modeled by a slab of nine layers, where the two topmost $\mathrm{W}$ layers have been fully relaxed. After relaxation the $\mathrm{W}-\mathrm{W}$ interlayer distance between the two topmost layers is reduced by $3.3 \%$, while the underneath $\mathrm{W}-\mathrm{W}$ interlayer distance increased by $1.1 \%$ compared to bulk W. A separating vacuum region of 18 $\AA$ width in the surface normal $(z)$ direction has been set up between neighboring supercell slabs. The average electron work function above the surface is calculated to be $\phi_{\mathrm{S}}=4.8 \mathrm{eV}$. A $41 \times 41 \times 5$ Monkhorst-Pack (MP) [110] $k$-point grid has been used for obtaining the orbitaldecomposed projected electron DOS onto the surface W atom, $n_{\mathrm{S} \alpha}^{a}(E)$. The same $k$-set has been used for calculating the sample electron wave functions for the TersoffHamann $[12,13]$ and Bardeen [14] simulations. The unit cell of the W(110) surface (shaded area) and the rectangular scan area for the tunneling current simulation are shown in Fig. 14. In the calculations, the experimental lattice constant $a_{W}=316.52 \mathrm{pm}$ has been used. Moreover, the surface top $(\mathrm{T})$ and hollow $(\mathrm{H})$ positions are explicitly shown.

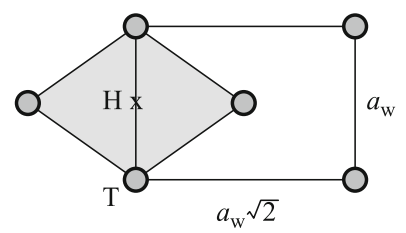

Fig. 14 The surface unit cell of W(110) (shaded area) and the rectangular scan area for the tunneling current simulations. Circles denote the $\mathrm{W}$ atoms. The top $(\mathrm{T})$ and hollow $(\mathrm{H})$ positions are explicitly shown. Reproduced from Ref. [91].

Different tip models were considered. The orbitalindependent ideal tip is characterized by $t_{\alpha \beta}=1$ and $n_{\mathrm{T} \beta}(E)=1 / 9(\mathrm{eV})^{-1}$, so that $n_{\mathrm{T}}(E)=\sum_{\beta} n_{\mathrm{T} \beta}(E)=$ $1(\mathrm{eV})^{-1}$. This ideal electronically flat tip represents the limiting case of the independent orbital approximation used in previous atom superposition tunneling models $[56,57,89,90]$. In order to study the effect of the orbital dependent tunneling, other tip models are needed. First, ideal tip models are considered, having a particular orbital symmetry $\beta_{0}$. In this case $t_{\alpha \beta}$ is calculated following Eq. (59), and for the energy dependence of the tip PDOS, $n_{T \beta_{0}}=1(\mathrm{eV})^{-1}$ and $n_{T\left(\beta \neq \beta_{0}\right)}=0$ are assumed. More realistic tips can also be employed by calculating the orbital decomposition of the tip apex PDOS in model tip geometries, and using Eq. (59) for the orbital depen- dent transmission factor. Let us consider a blunt W(110) tip. Motivated by a previous simulation [114], it has been modeled by a slab consisting of three atomic layers having one $\mathrm{W}$ apex atom on both surfaces, i.e., with a double vacuum boundary. In this system the apex atoms have been relaxed on both sides. The adatom-topmost layer vertical distance decreased by $19.3 \%$ compared to bulk $\mathrm{W}$. The interaction between apex atoms in neighboring supercells in the lateral direction is minimized by choosing a $3 \times 3$ surface cell, and a $17.9 \AA$ wide separating vacuum region in the $z$ direction. The local electron work function above the tip apex was assumed to be $\phi_{\mathrm{T}}=4.8$ $\mathrm{eV}$. Moreover, an $11 \times 15 \times 5 \mathrm{MP} k$-point grid has been chosen for calculating the orbital-decomposed projected DOS onto the apex atom, $n_{\mathrm{T} \beta}(E)$. The same $k$-point sampling has been used for obtaining the tip electron wave functions for the Bardeen calculation.

STM images were simulated employing the orbital dependent 3D WKB tunneling model, and the TersoffHamann $[12,13]$ and Bardeen [14] methods implemented in the BSKAN code $[4,115]$. Scattering up to first order [115] did not affect the quality of the images. Using the orbital dependent model, the tunneling current has been calculated in a box above the rectangular scan area shown in Figure 14 containing $99000(30 \times 22 \times 150)$ grid points with a $0.149 \AA$ lateral and $0.053 \AA$ vertical resolution. The electron local density of states (LDOS) was calculated above the same scan area in a box of $31 \times 21 \times 101$ grid points using the Tersoff-Hamann method with the same spatial resolution as reported above. For the calculation of the tunneling current employing the Bardeen method, a box of $31 \times 10 \times 100$ grid points above the half of the rectangular scan area has been chosen in order to speed up the simulation. In this case the lateral resolution remains $0.149 \AA$, and the vertical resolution is 0.106 A. The constant current contours are extracted following the method described in Section 2.4.1. All of the STM images will be presented above the full rectangular scan area.

\subsection{Convergence properties}

Previously, the convergence of the $\mathrm{d} I / \mathrm{d} U$ part of the differential conductance has been investigated with respect to the number of surface atoms involved in the summation of the orbital-independent atomic superposition formula [89]. Due to the spherical exponential decay assumed for the electron wave functions, a rapid convergence was found. Here, a similar convergence test for the orbital-dependent tunneling approach is reported, comparing different tip models. In order to take into account a wide energy range around the Fermi level, the tunneling current has been calculated at $-2.5 \mathrm{~V}$ and +2.5 
$\mathrm{V}$ bias voltages at $z=4.5 \AA$ above a surface $\mathrm{W}$ atom, and these current values have been averaged. The following tips were considered: the ideal tip of the orbitalindependent model, ideal tips with $s, p_{z}$, and $d_{3 z^{2}-r^{2}}$ symmetry, and the W(110) tip. In order to obtain comparable results, the averaged current was normalized for each tip calculation. The convergences of the normalized averaged current with respect to the lateral distance on the surface, $d_{/ /}$, characteristic for the number of atoms involved in the atomic superposition, are shown in Fig. 15. By calculating the current, contributions from surface atoms within a radius of $d_{/ /}$measured from the $\mathrm{W}$ atom below the tip apex are summed up (sum over $a$ ). It is found that the orbital-independent, the $s$-type, and the $\mathrm{W}(110)$ tips behave quite similarly concerning the current convergence, while for the $p_{z^{-}}$and $d_{3 z^{2}-r^{2} \text {-type }}$ tips a faster convergence is found. This rapid convergence can be explained by the more localized character of the latter tip orbitals in the direction normal to the sample surface $(z)$. On the other hand, the orbitalindependent tip with $T=\mathrm{e}^{-2 \kappa d}$ is a good approximation for the $s$-type tip (with index $\beta=1$ ), where the spherically decaying transmission function part is still dominant, i.e., $T_{\alpha, 1}=\mathrm{e}^{-2 \kappa d} \chi_{\alpha}^{2}$ because $\chi_{1}^{2}=1$. In case of the $\mathrm{W}(110)$ tip, electronic states of all considered symmetries have a contribution, and their relative importance is not only determined by the transmission function via the orbital shapes but also by the product of the symmetrydecomposed electron PDOS of the surface and the tip. In general, the orbitals localized in different than the $z$ direction can show a slower current convergence than the $s$ orbitals. However, the partial PDOS of such states is relatively low, and interestingly, a similar current convergence is obtained in the studied energy range as for the $s$-type tip. Choosing different bias voltages for the $\mathrm{W}(110)$ tip, thus different electron states involved in the tunneling, current convergences dissimilar to the $s$-type

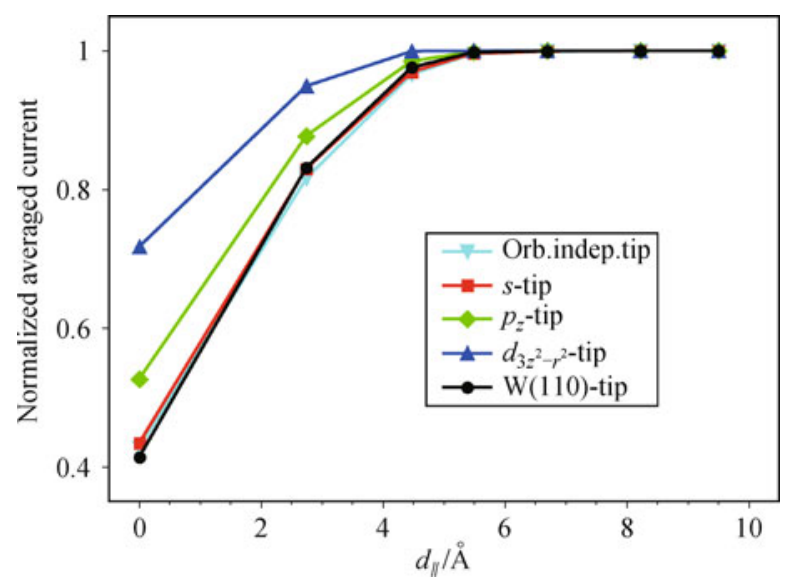

Fig. 15 Convergence of the normalized averaged current calculated with different tip models. Reproduced from Ref. [91]. tip behavior were found. The convergence can be slower or faster than obtained for the $s$-type tip depending on the partial PDOS of each directional orbital in the given energy range.

Based on the convergence tests, atom contributions within at least $d_{/ /}=3 a \approx 9.5 \AA$ distance from the surface-projected tip position shall be considered. In case of calculating STM images, $d_{/ /}=3 a \approx 9.5 \AA$ has to be measured from the edge of the scan area in all directions in order to avoid distortion of the image, thus involving 67 surface atoms in the atomic superposition. For brevity, in the following, the same surface atoms are used in order to calculate single point tunneling properties as well.

\subsection{Orbital contributions}

Let us analyze the relative importance of all $\alpha \leftrightarrow \beta$ transitions in determining the total tunneling current at different tip positions. From this analysis, a qualitative picture about the role of the different atomic orbitals in the construction of the tunneling current is obtained. The $I_{\alpha \beta}$ current contributions can be calculated according to Eq. (62). These can be represented by a current histogram that gives the percentual contributions of the individual transitions to the total current. Figure 16 shows such histograms using the W(110) tip at $V=-0.1 \mathrm{~V}$ bias voltage $z=4.5 \AA$ above two different tip positions: part a) corresponds to the tip apex above the surface top position, and part b) to the tip apex above the surface hollow position, $\mathrm{T}$ and $\mathrm{H}$ in Fig. 14, respectively. A $9 \times 9$ matrix from the considered orbitals is obtained, where the orbitals are denoted by numbers 1 to 9 following the indices reported in Table 2. One finds that most contributions are due to the $s(1), p_{z}(3), d_{y z}$ (6), $d_{3 z^{2}-r^{2}}(7)$, and $d_{x z}(8)$ orbitals and their combinations. The largest contribution to the current is given by the $d_{3 z^{2}-r^{2}}-d_{3 z^{2}-r^{2}}(7-7)$ transition, 31 and 20 per cent above the top and hollow positions, respectively. Concomitantly, above the hollow position, the relative importance of both tip and sample $d_{y z}(6)$ and $d_{x z}$ (8) orbitals is increased as it is expected from the geometrical setup, i.e., the $d_{y z}-d_{y z}(6-6), d_{y z}-d_{3 z^{2}-r^{2}}(6-7$ and $7-6), d_{x z}-d_{x z}(8-8)$, and $d_{x z}-d_{3 z^{2}-r^{2}}(7-8$ and $8-7)$ contributions correspond to larger orientational overlap of the mentioned tip and sample orbitals if the tip is above the hollow position rather than above the top position as suggested by the geometry in Fig. 14 and Eq. (59). For more theoretical insights, please see Ref. [116], where the concept of a parallel path of conduction has been introduced. Thus, the orbital dependent 3D WKB tunneling model captures the effect of the localized orbitals and goes beyond the spherical Tersoff-Hamann model. 
(a)

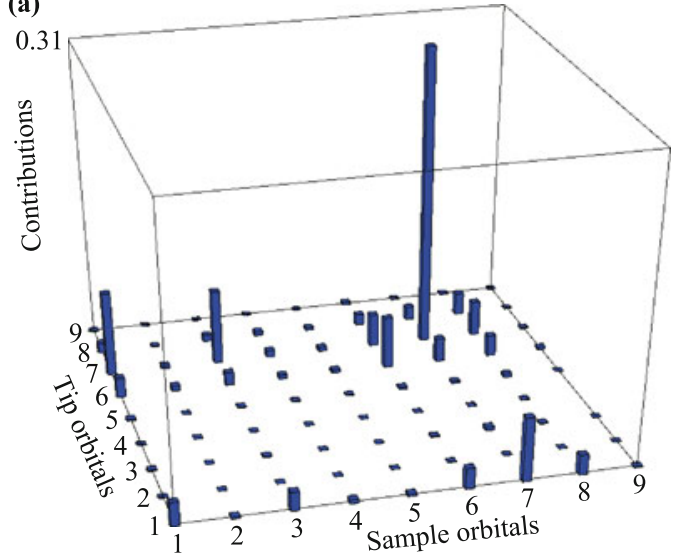

(b)

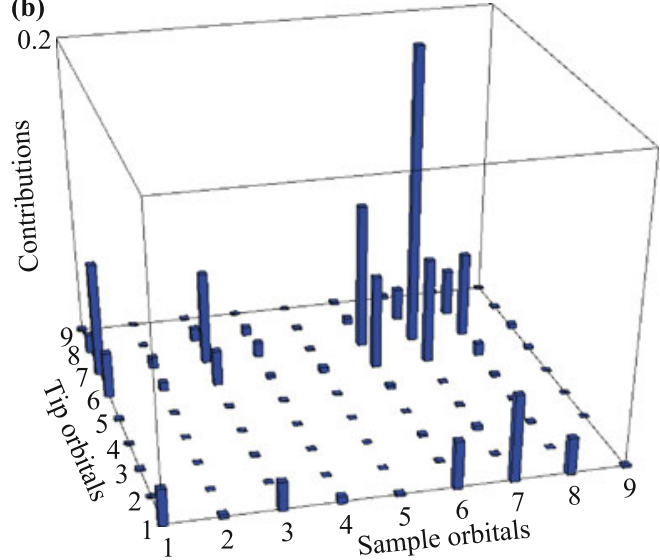

Fig. 16 Histograms of the current contributions $\left(I_{\beta \gamma}\right)$ from all tip-sample transitions with different orbital symmetries using the $\mathrm{W}(110)$ tip at $V=-0.1 \mathrm{~V}$ bias. (a) Tip apex $z=4.5 \AA$ above the surface top (T) position (W atom); (b) tip apex $z=4.5 \AA$ above the surface hollow $(\mathrm{H})$ position, see also Fig. 14. The indices of the atomic orbitals (1-9) follow the notation reported in Table 2. Reproduced from Ref. [91].

Note that if a larger bias voltage is considered, i.e., the electronic states are somewhat averaged, then the independent orbital approach might turn out to be a good approximation [90].

\subsection{Atomic contrast reversal}

The role of the localized orbitals can best be demonstrated by reinvestigating the corrugation inversion phenomenon found, e.g., on (100) [24], (110) [112], and (111) [117] metal surfaces. Chen explained this effect as a consequence of $m \neq 0$ tip states [23]. According to Heinze et al. [112], under certain circumstances the apparent height of $\mathrm{W}$ atoms at the surface top position $\left(z_{\mathrm{T}}\right)$ can be larger or smaller than the apparent height of the surface hollow position $\left(z_{\mathrm{H}}\right)$ at constant current ( $I=$ const) condition. (For the surface top $(\mathrm{T})$ and hollow $(\mathrm{H})$ positions, see Fig. 14.) Thus, the sign change of $\Delta z(I)=z_{\mathrm{T}}(I)-z_{\mathrm{H}}(I)$ is indicative for the corrugation inversion. Obviously, $\Delta z(I)>0$ corresponds to a normal STM image, where the $\mathrm{W}$ atoms appear as protrusions, and $\Delta z(I)<0$ to an anticorrugated image. Since the tunneling current is monotonically decreasing with the increasing tip-sample distance, one can obtain information about the occurrence of the corrugation inversion simply by calculating the current difference between tip positions above the top and hollow sites of the W(110) surface. The current difference at tip-sample distance $z$ and at bias voltage $V$ is defined as

$$
\Delta I(z, V)=I_{\mathrm{T}}(z, V)-I_{\mathrm{H}}(z, V)
$$

This quantity can be calculated for specific tips, and the $\Delta I(z, V)=0$ contour is called the corrugation inversion map [91]. This gives the $(z, V)$ combinations where the corrugation inversion occurs. The sign of $\Delta I(z, V)$ pro- vides the corrugation character of an STM image in the given $(z, V)$ regime. Due to the monotonically decreasing character of the tunneling current, $\Delta I(z, V)>0$ corresponds to $\Delta z(I(V))>0$, i.e., normal corrugation, and similarly $\Delta I(z, V)<0$ corresponds to $\Delta z(I(V))<0$ and anticorrugation.

First, $\Delta I(z, V)$ was calculated using the independent orbital approximation and Eq. (12) for the tunneling transmission, and $\Delta I(z, V)$ was found always positive. This means that the spherical exponential decay itself can not account for the observed corrugation inversion effect, and the $\mathrm{W}$ atoms always appear as protrusions in STM images calculated with this model. However, considering the orbital dependent tunneling transmission in Eq. (55) evidence is found for the corrugation inversion effect, thus highlighting the role of the real space shape of electron orbitals involved in the tunneling. Figure 17 shows $\Delta I(z, V)=0$ contours calculated with different tip models in the $[0 \AA, 14 \AA]$ tip-sample distance and $[-2 \mathrm{~V},+2 \mathrm{~V}]$ bias voltage range. Before turning to the analysis of the results obtained with previously not considered tip models let us compare the results with those of Heinze et al. [112], where an $s$-wave tip model has been used. They found corrugation reversal at around $-0.4 \mathrm{~V}$ at $z=4.6 \AA$ tip-sample distance, and above that voltage normal while below anticorrugated STM images were obtained. The orbital dependent 3D WKB model with an $s$-tip provides the same type of corrugation reversal at $-0.21 \mathrm{~V}$ at the same distance as can be seen in part a) of Fig. 17 (curve with filled square symbol). These bias values are in reasonable agreement particularly concerning their negative sign. At this range atomic resolution is difficult to achieve experimentally, which is an indication for being close to the corrugation inversion regime [112]. On the other hand a linear dependence of 

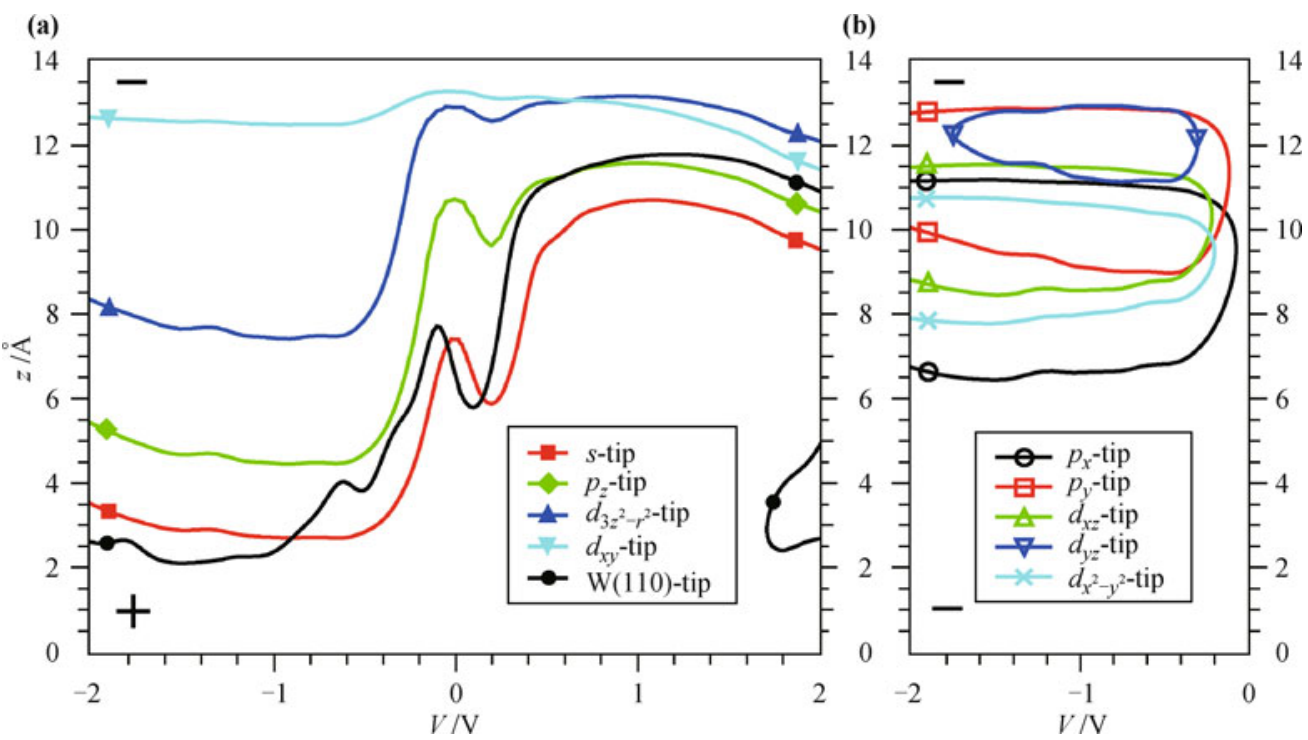

Fig. 17 The $\Delta I(z, V)=I_{\mathrm{T}}(z, V)-I_{\mathrm{H}}(z, V)=0$ contours indicative for the corrugation inversion [see Eq. (88), and its meaning in the text] calculated with different tip models above the W(110) surface. Parts (a) and (b) show two distinct behaviors depending on the tip orbital composition. The sign of $\Delta I(z, V)$ is explicitly shown: In part (a) it is positive (+) below the curves, and negative (-) above them; in part (b) positive inside the loop of a given curve, and negative ( - ) outside the loop. Note that positive $\Delta I(z, V)$ corresponds to normal, while negative to inverted atomic contrast. Reproduced from Ref. [91].

the corrugation reversal voltage and the tip-sample distance has been reported by Heinze et al. $(z=4.6 \AA$, $V=-0.4 \mathrm{~V})$ to $(z=7.2 \AA, V=0 \mathrm{~V})$. Our model qualitatively reproduces this linear dependence in the same bias range though the quantitative values are somewhat different.

Calculating the corrugation inversion maps with more tip models, two distinct behaviors depending on the tip orbital composition were found. Parts (a) and (b) of Fig. 17 show these. While the tip models in part (a) can show corrugation inversion in the whole studied bias range, this effect does not occur at positive bias voltages for tips in part (b). Moreover, anticorrugation $(\Delta I(z, V)<0)$ is observed in the large tip-sample distance region $(z>13.5 \AA)$ in both parts. This is in accordance with the prediction of Ref. [113] based on the analysis of the competing electron states in the surface Brillouin zone of an $\mathrm{Fe}(001)$ surface. In the $z<13.5 \AA$ range, however, the corrugation character in the two parts of Fig. 17 is remarkably different. In part (a), normal corrugation is found close to the surface, which reverts only once with increasing tip-sample distance for the tip models with a single orbital symmetry in the full studied bias range. The $\mathrm{W}(110)$ tip behaves similarly below +1.7 $\mathrm{V}$, while above there is a double reversal of the corrugation character as the tip-sample distance increases. This indicates that anticorrugation can be expected at short tip-sample distances $(3.5 \AA-5 \AA)$ at around +2 $\mathrm{V}$. On the other hand, the tip models in part (b) always show anticorrugation at positive bias voltages, and below
$-0.05 \mathrm{~V}$ they provide corrugation characters starting from anticorrugation, then normal corrugation, and again anticorrugation with increasing tip-sample distance. These different behaviors can be attributed to the tip orbital characters. It is interesting to notice that none of the considered tip orbitals in part b) are localized in the $z$-direction, and they have nodal planes either in the $y z$ plane $\left(p_{x}\right.$ and $\left.d_{x z}\right)$ or in the $x z$ plane $\left(p_{y}\right.$ and $\left.d_{y z}\right)$ or in the $x=y$ and $x=-y$ planes $\left(d_{x^{2}-y^{2}}\right)$. On the other hand, in part a) there are tips which are localized in the $z$-direction $\left(p_{z}\right.$ and $d_{3 z^{2}-r^{2}}$ ) or having nodal planes in both the $x z$ and $y z$ planes $\left(d_{x y}\right)$ as well as the spherical $s$ tip and the W(110) tip that contains all type of orbitals with energy dependent partial PDOS functions. The particular tip nodal planes restrict the collection of surface atom contributions to specific regions on the sample surface. Furthermore, by changing the tip-sample distance, the orientational overlaps between the tip and sample orbitals change, and according to the model some localized orbitals gain more importance in the tunneling contribution, see also Fig. 16. Since the current difference is calculated between tip positions above the surface top and hollow sites, the complex tip-sample and bias voltage dependent effect of the real space orbitals on the tunneling can be visualized via the corrugation inversion maps.

Concerning tips with $p_{z}$ and $d_{3 z^{2}-r^{2}}$ orbital symmetry, Heinze et al. [112] calculated a corrugation enhancement factor of 2 and 6.25 , respectively, based on Chen's derivative rule [21]. Moreover, they argued that the corrugation 
inversion map should be practically identical to the one obtained by using the $s$-tip model, and the corrugation values just have to be scaled up by these factors. On the contrary, based on the orbital dependent 3D WKB tunneling model one finds that the $p_{z}$ and $d_{3 z^{2}-r^{2}}$ tips provide qualitatively different corrugation inversion maps, i.e., although their bias dependent shape is similar to the one of the $s$-tip, their tip-sample distance is systematically pushed to larger values, see part a) of Fig. 17 . This is due to the more localized character of these tip orbitals in the $z$-direction.

Corrugation inversion with the $d_{x y}$ tip occurs at the largest tip-sample distance. A possible explanation can be based on its $x z$ and $y z$ nodal planes. While above the top position only the underlying $\mathrm{W}$ atom, above the hollow position all four nearest neighbor $\mathrm{W}$ atoms give zero contribution to the current, thus $I_{\mathrm{T}}$ is expected to be higher than $I_{\mathrm{H}}$ at small tip-sample distances. To overcome this effect the tip has to be moved farther from the surface since then the relative importance of the nearest neighbor contributions decays rapidly compared to other parts of the surface.

Apart from above findings, corrugation inversion is also obtained in the positive bias range at enlarged tipsample distances for the $s, p_{z}, d_{3 z^{2}-r^{2}}$, and $\mathrm{W}(110)$ tips considered in part (a) of Fig. 17. This is most probably due to the surface electronic structure. Note that this effect is even more difficult to capture in experiments as the corrugation values themselves decay rapidly with increasing tip-sample distance [57].

\subsection{STM images - Comparison to other tunneling models}

In order to demonstrate the corrugation inversion more apparently, constant current STM images can be simulated. As it is clear from Fig. 17, any type of crossing of the $\Delta I(z, V)=0$ contour results in the occurrence of the corrugation reversal. In experiments two ways can be considered to record STM images in the normal and anticorrugated regimes: i) keep the tip-sample distance $z$ constant, and change the bias voltage $V$; or ii) keep the bias voltage $V$ constant, and change the tip-sample distance. Respectively, these modes correspond to a horizontal and a vertical crossing of the $\Delta I(z, V)=0$ contour in the $(z, V)$ plane in Fig. 17. Heinze et al. followed the first method in their simulations [112]. However, as the second option seems to be experimentally more feasible and needs less calculations as well, STM images were simulated at a fixed bias voltage of $-0.25 \mathrm{~V}$ [91].

In Fig. 18, STM images are compared using the orbital dependent 3D WKB model assuming an $s$-type tip [first row (a)-(c)] to those calculated by the Tersoff-Hamann method [second row (d)-(f)]. One finds that the images are in good qualitative agreement for the (a)-(d), (b)(e), and (c)-(f) pairs, respectively. In parts (a) and (d), at a tip-sample distance of about $3.80 \AA$, the apparent height of the $\mathrm{W}$ atom is larger than the one of the hollow position, i.e., $\Delta z=z_{\mathrm{T}}-z_{\mathrm{H}}>0$. This resembles normal corrugation. Moving the tip farther from the surface, the corrugation inversion and striped images are obtained. These are shown in parts (b) and (e) of Fig. 18. It is found that the orbital dependent 3D WKB method reproduces the corrugation inversion effect at almost the same tipsample distance $(4.15 \AA)$ as the Tersoff-Hamann model predicts $(4.21 \AA)$. Increasing the tip-sample distance further, the anticorrugation regime is reached, and the apparent height of the $\mathrm{W}$ atom is smaller than the one of the hollow position, i.e., $\Delta z=z_{\mathrm{T}}-z_{\mathrm{H}}<0$. Such images are shown in parts (c) and (f). Note that all of the simulated STM images in Figure 18 are in good qualitative agreement with Ref. [112]. The corrugation of the individual current contours has also been calculated: (a) $\Delta z^{\prime}=0.23 \mathrm{pm}$, (b) $\Delta z^{\prime}=0.10 \mathrm{pm}$, (c) $\Delta z^{\prime}=0.12 \mathrm{pm}$, (d) $\Delta z^{\prime}=1.63 \mathrm{pm}$, (e) $\Delta z^{\prime}=1.82 \mathrm{pm}$, (f) $\Delta z^{\prime}=1.79 \mathrm{pm}$. One finds that the orbital dependent 3D WKB model gives approximately one order of magnitude less corrugation than the Tersoff-Hamann method. Note, however, that the small corrugation amplitudes using the orbital dependent 3D WKB method are in good agreement with the results of Ref. [112], where $\Delta z^{\prime}<1$ $\mathrm{pm}$ is reported close to the contrast reversal.

As we have seen, the corrugation inversion effect already occurs considering the electronic structure of the sample only. However, Fig. 17 indicates that different tips can modify its tip-sample distance and bias voltage dependence quite dramatically. In Fig. 19 STM images are compared using the orbital dependent 3D WKB model [first row (a)-(c)] to those calculated by the Bardeen method [second row (d)-(f)] explicitly taking the electronic structure of the $\mathrm{W}(110)$ tip in both cases into account. One finds that the images are in good qualitative agreement for the (b)-(e) and (c)-(f) pairs. In parts (a) and (d), at a tip-sample distance of about $4.50 \AA$, the agreement is weaker, however, the normal corrugation is more pronounced in the orbital dependent $3 \mathrm{D} \mathrm{WKB}$ model: The corrugation amplitude of part (a) $\Delta z^{\prime}=0.36$ $\mathrm{pm}$ is much larger than that of part (d) $\Delta z^{\prime}=0.07 \mathrm{pm}$. Note that employing the orbital dependent $3 \mathrm{D}$ WKB model, a better qualitative agreement to the image of part (d) has been found at a larger tip-sample separation, i.e., closer to the corrugation inversion. This inversion is demonstrated in parts (b) and (e) of Fig. 19. Again, striped images are obtained. Note, however, that the stripes with larger apparent height correspond to the atomic rows, in contrast to what has been found in 

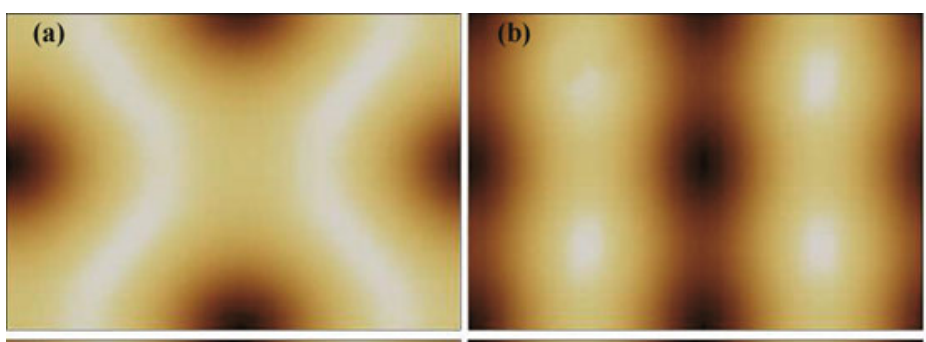

(d)

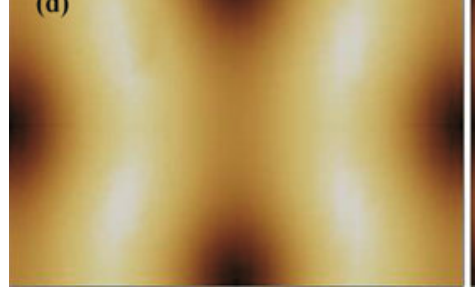

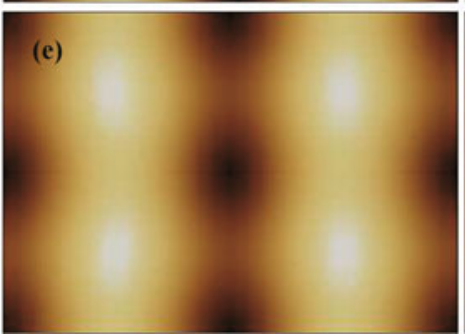

Fig. 18 Comparison of simulated STM images of the W(110) surface using the orbital dependent 3D WKB model with an $s$-type tip [top, (a)-(c)] and the Tersoff-Hamann model [bottom, (d)-(f)] at $V=-0.25 \mathrm{~V}$ bias voltage. The scan area corresponds to the rectangle shown in Fig. 14. Light and dark areas denote higher and lower apparent heights, respectively. The apparent heights of the $\mathrm{W}$ atom $\left(z_{\mathrm{T}}\right)$, and the corrugation of the contours $\left(\Delta z^{\prime}\right)$ are as follows: Orbital dependent 3D WKB model: (a) $z_{\mathrm{T}}=3.80 \AA, \Delta z^{\prime}=0.23 \mathrm{pm}$; (b) corrugation inversion, $z_{\mathrm{T}}=4.15 \AA, \Delta z^{\prime}=0.10 \mathrm{pm}$; (c) $z_{\mathrm{T}}=4.35$ $\AA, \Delta z^{\prime}=0.12 \mathrm{pm}$. Tersoff-Hamann model: (d) $z_{\mathrm{T}}=3.80 \AA, \Delta z^{\prime}=1.63 \mathrm{pm}$; (e) corrugation inversion, $z_{\mathrm{T}}=4.21 \AA$, $\Delta z^{\prime}=1.82 \mathrm{pm}$; (f) $z_{\mathrm{T}}=4.70 \AA, \Delta z^{\prime}=1.79 \mathrm{pm}$. Reproduced from Ref. [91].

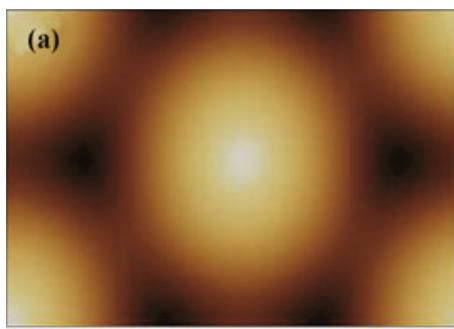

(d)

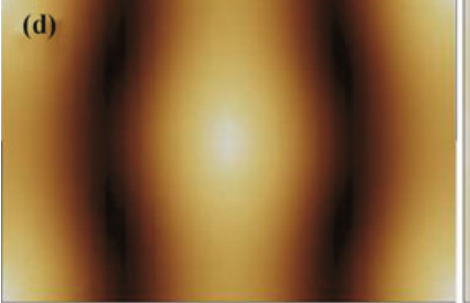

(b)

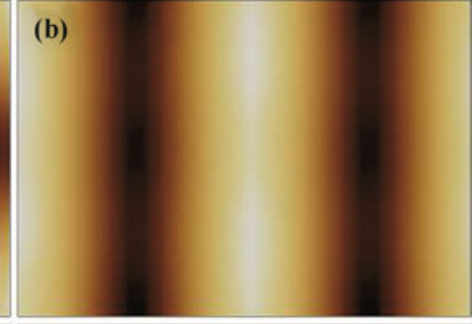

(e)

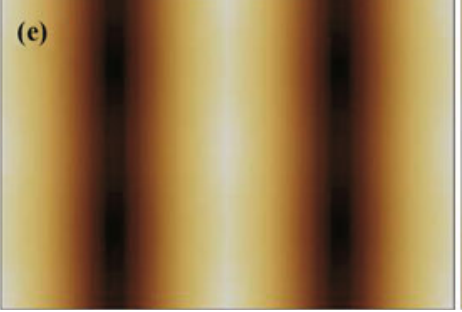

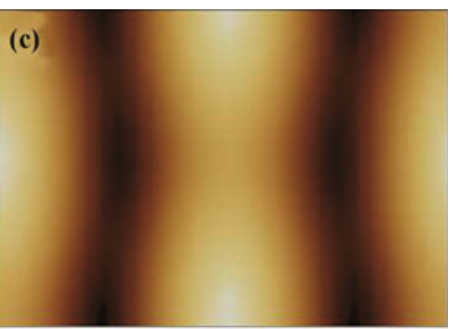

(f)

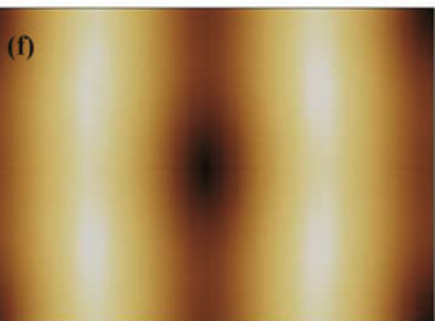

(f)

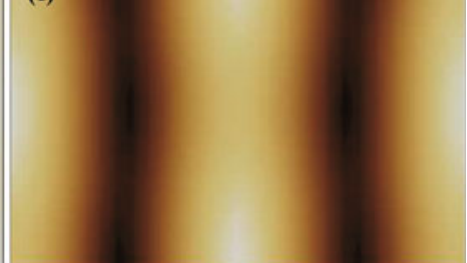

Fig. 19 Comparison of simulated STM images of the W(110) surface using the orbital dependent 3D WKB model [top, (a)-(c)] and the Bardeen method [bottom, (d)-(f)] with the $\mathrm{W}(110)$ tip at $V=-0.25 \mathrm{~V}$ bias voltage. The scan area corresponds to the rectangle shown in Fig. 14. Light and dark areas denote higher and lower apparent heights, respectively. The current values $(I)$, the apparent heights of the $\mathrm{W}$ atom $\left(z_{\mathrm{T}}\right)$, and the corrugation of the contours $\left(\Delta z^{\prime}\right)$ are as follows: Orbital dependent 3D WKB model: (a) $I=6.3 \mathrm{nA}, z_{\mathrm{T}}=4.50 \AA, \Delta z^{\prime}=0.36 \mathrm{pm}$; (b) corrugation inversion, $I=0.43 \mathrm{nA}$, $z_{\mathrm{T}}=5.80 \AA, \Delta z^{\prime}=0.02 \mathrm{pm} ;$ (c) $I=0.35 \mathrm{nA}, z_{\mathrm{T}}=5.90 \AA, \Delta z^{\prime}=0.02 \mathrm{pm}$. Bardeen method: (d) $I=4.4 \mathrm{nA}, z_{\mathrm{T}}=4.50$ $\AA, \Delta z^{\prime}=0.07 \mathrm{pm}$; (e) corrugation inversion, $I=0.7 \mathrm{nA}, z_{\mathrm{T}}=5.55 \AA, \Delta z^{\prime}=0.03 \mathrm{pm}$; (f) $I=0.19 \mathrm{nA}, z_{\mathrm{T}}=6.25 \AA$, $\Delta z^{\prime}=0.02$ pm. Reproduced from Ref. [91].

parts (b) and (e) of Fig. 18, where the atomic and hollow sites appeared as depressions. This difference is definitely due to the effect of the $\mathrm{W}$ tip, which was not considered in Fig. 18. On the other hand, one finds good agreement concerning the tip-sample distance of the corrugation inversion: $5.80 \AA$ in the orbital dependent 3D WKB model, and $5.55 \AA$ calculated by the Bardeen method. Parts (c) and (f) of Fig. 19 correspond to anticorrugated images. In this tip-sample distance regime the extremely small corrugation amplitudes are in good agreement between the orbital dependent 3D WKB model and the Bardeen method: $\Delta z^{\prime}=0.02 \mathrm{pm}$ in parts (b), (c), (f), and $\Delta z^{\prime}=0.03 \mathrm{pm}$ in part (e). Note that for the current calculations within the $3 \mathrm{D}$ WKB approach, $\varepsilon=1 \mathrm{eV}$ has been used in Eq. (31), that provides comparable current values to the Bardeen results.

Finally, computational times between the orbital dependent 3D WKB model and the Bardeen method were 
compared, and the following was found:

i) orbital dependent model, $30 \times 22 \times 150$ grid points, $1 \mathrm{CPU}$, time $=229 \mathrm{~s}$;

ii) Bardeen method in BSKAN code, $31 \times 10 \times 100$ grid points, 4 CPUs, time $=173866 \mathrm{~s}$.

Normalizing to the same real space grid points, one obtains that the orbital dependent 3D WKB method is 2425 times faster using one CPU than using four CPUs for the Bardeen calculation. As the four CPUs' calculations are approximately 3.5 times faster than the calculations on one CPU in standard computer clusters, a remarkable one-CPU equivalent time boost of about 8500 is obtained for the orbital dependent 3D WKB method compared to the Bardeen method for the given surfacetip combination. While the $k$-point samplings of the surface and tip Brillouin zones affect the computational time of the Bardeen method due to the enhanced number of transitions as the number of $k$-points increases, the computational time of the orbital dependent 3D WKB model is insensitive to the number of $k$-points as the PDOS of the tip apex and surface atoms are used. The energy dependent PDOS functions have the same data structure, no matter of the number of the constituting electron states obtained by the $k$-summation [56]. This is a great computational advantage in favor of the orbital dependent 3D WKB model. Of course, the quality of the results depends very much on the $k$-point samplings.

\section{Summary and outlook}

In this work, we reviewed the recently developed 3D WKB atom-superposition approach for simulating STM and STS, based on electronic structure data obtained from first principles. In the method, contributions from individual electron tunneling transitions between the tip apex atom and each of the sample surface atoms are summed up assuming the 1D WKB approximation in all these transitions. This tunneling model suits well to simulate spin-polarized STM and STS on surfaces exhibiting a complex noncollinear magnetic structure, i.e., without a global spin quantization axis, at very low computational cost. The tip electronic structure from first principles can also be incorporated into the model, that is often assumed to be constant in energy in the vast majority of the related literature, which could lead to a misinterpretation of experimental findings. Using this approach, we highlighted some of the electron tunneling features on a prototype frustrated hexagonal antiferromagnetic Cr monolayer on $\operatorname{Ag}(111)$ surface. We obtained useful theoretical insights into the simulated quantities that is expected to help the correct evaluation of experimental results, even on different magnetic surfaces. By extend- ing the method to incorporate a simple orbital dependent electron tunneling transmission, we reinvestigated the bias voltage- and tip-dependent contrast inversion effect on the W(110) surface. We highlighted the role of the real space shape of the orbitals involved in the tunneling, rather than considering electron states in the reciprocal space, thus a different kind of understanding is provided. STM images calculated using this orbital dependent model agree reasonably well with Tersoff-Hamann and Bardeen results.

The main advantages of the 3D WKB method are computational efficiency, i.e., computational time and data storage throughout the simulation, and the flexibility of using any electronic structure code for the PDOS inputs. So far, the method has been tested and used employing the PDOS calculated by the VASP code [103105], the SKKR code [118, 119], and the FLEUR code (http://www.flapw.de, tip PDOS data calculated by Ferriani et al. [76]). It is possible to combine different levels of electronic structure calculations for the sample surface and the tip, see, e.g., Ref. [10]. The most important feature is that the $k$-point samplings of the surface and tip Brillouin zones do not affect the computational time, in contrast to the Bardeen method. We showed that in a certain case, a relative computational time gain of 8500 is obtained compared to the Bardeen calculation, without the loss of quality. On the other hand, calculating and storing the projected DOS onto surface atoms is computationally much cheaper compared to the exact vacuum LDOS of the surface on a great number of real space grid points: While the $n_{\mathrm{S}}^{a}(E)$ and $\boldsymbol{m}_{\mathrm{S}}^{a}(E)$ real quantities take altogether at most 60 kBytes on a 5101 $E$-point grid in the three-atomic magnetic unit cell of the $\mathrm{Cr} / \operatorname{Ag}(111)$ surface, the $n_{\mathrm{S}}(x, y, z, E)$ and $\boldsymbol{m}_{\mathrm{S}}(x, y, z, E)$ real quantities in Eqs. (6) and (7) take altogether 2.9 GBytes on the same $E$-grid in the box above the magnetic unit cell containing $153000(34 \times 30 \times 150)$ real space grid points. Moreover, the atom-projected PDOS functions are routinely obtained in all $a b$ initio electronic structure codes, whereas the vacuum LDOS is not always routinely accessible for the average user. Thus the presented 3D WKB method meets the requirement for an efficient user-friendly simulation tool that could be easily used by experimental groups, and give (SP-)STM and (SP-)STS results within minutes with reasonable accuracy.

The picture would not be complete without mentioning the limitations of the 3D WKB method. For example, it does not account for multiple scattering [115] and interference effects [24,31], which could be important for certain systems. Therefore, it is expected that the 3D WKB model works well on simple metals, possibly on molecular adsorbates on surfaces as well, but not on 
materials with strong band structure or Fermi surface effects. Another limitation is the uniform vacuum decay $\kappa$ of the electron states for different orbital symmetries in Eq. (55). At the moment, the orbital effects are contained in the geometry factor, Eq. (59) only. Still, the model in its present form provides comparable results to more sophisticated tunneling models (Tersoff-Hamann, Bardeen), see Section 4.5.

Though some of these limitations cannot be overcome owing to the simple physical picture behind the $3 \mathrm{D}$ WKB method, other limitations call for further extensions of the model, to become an even more powerful tool for (SP-)STM and (SP-)STS simulations. One type of improvements concerns the tunneling transmission function. Considering different decays for different orbital symmetries, the vacuum decay $\kappa$ in Eq. (55) could also be described depending on the electron orbitals. Furthermore, the correct description of the electron work function is extremely important on strongly corrugated surfaces [102]. Related to this, the sample work function can be position-dependent, $\phi_{\mathrm{S}}(x, y)$, or even positionand orientation-dependent, $\phi_{\mathrm{S}}(x, y, \vartheta, \varphi)$. Here, the angles can be calculated in a fixed tip position. Using such improved local work functions, the description of the effective rectangular tunnel barrier can be replaced by more sophisticated tunnel barrier models. Moreover, extending the orbital dependent $3 \mathrm{D}$ WKB model to magnetic junctions is expected to enable the study of the interplay of real space orbital and spin polarization effects in SP-STM and SP-STS experiments in the future. On the other hand, the orbital dependent 3D WKB model makes it possible to consider the effect of arbitrary crystallographic tip orientations on the tunneling properties. The more sophisticated tunneling models are very restricted to treat such effects at high computational cost [114]. Owing to the atom-superposition framework, the effect of multi-tip apices or nanotips can also be studied that have experimental relevance since the tip preparation techniques are hard to control [120]. Finally, the real space character of the 3D WKB method to calculate tunneling properties on a fine grid in a box above the surface enables the further speed-up of the calculations by parallelizing the code via the partitioning of the real space box.

Acknowledgements The authors thank L. Szunyogh, S. Heinze, P. Ferriani, G. Teobaldi, and C. Panosetti for useful discussions. We thank P. Ferriani and S. Heinze for providing us a set of tip PDOS data calculated by the FLEUR code. Financial support of the Magyary Foundation, EEA and Norway Grants, the Hungarian Scientific Research Fund (OTKA PD83353, K77771), the Bolyai Research Grant of the Hungarian Academy of Sciences, and the New Széchenyi Plan of Hungary (Project ID: TÁMOP-4.2.2.B10/1-2010-0009) is gratefully acknowledged.

\section{References}

1. G. Binnig, H. Rohrer, C. Gerber, and E. Weibel, Tunneling through a controllable vacuum gap, Appl. Phys. Lett., 1982, 40(2): 178

2. G. Binnig, H. Rohrer, C. Gerber, and E. Weibel, Surface studies by scanning tunneling microscopy, Phys. Rev. Lett., 1982, 49(1): 57

3. W. A. Hofer, A. S. Foster, and A. L. Shluger, Theories of scanning probe microscopes at the atomic scale, Rev. Mod. Phys., 2003, 75(4): 1287

4. W. A. Hofer, Challenges and errors: Interpreting high resolution images in scanning tunneling microscopy, Prog. Surf. Sci., 2003, 71(5-8): 147

5. V. A. Ukraintsev, Data evaluation technique for electrontunneling spectroscopy, Phys. Rev. B, 1996, 53(16): 11176

6. B. Koslowski, C. Dietrich, A. Tschetschetkin, and P. Ziemann, Evaluation of scanning tunneling spectroscopy data: Approaching a quantitative determination of the electronic density of states, Phys. Rev. B, 2007, 75(3): 035421

7. M. Passoni, F. Donati, A. Li Bassi, C. S. Casari, and C. E. Bottani, Recovery of local density of states using scanning tunneling spectroscopy, Phys. Rev. B, 2009, 79(4): 045404

8. M. Ziegler, N. Néel, A. Sperl, J. Kröger, and R. Berndt, Local density of states from constant-current tunneling spectra, Phys. Rev. B, 2009, 80(12): 125402

9. B. Koslowski, H. Pfeifer, and P. Ziemann, Deconvolution of the electronic density of states of tip and sample from scanning tunneling spectroscopy data: Proof of principle, Phys. Rev. B, 2009, 80(16): 165419

10. K. Palotás, W. A. Hofer, and L. Szunyogh, Simulation of spin-polarized scanning tunneling spectroscopy on complex magnetic surfaces: Case of a $\mathrm{Cr}$ monolayer on $\mathrm{Ag}(111)$, Phys. Rev. B, 2012, 85(20): 205427

11. M. Brandbyge, J. L. Mozos, P. Ordejón, J. Taylor, and K. Stokbro, Density-functional method for nonequilibrium electron transport, Phys. Rev. B, 2002, 65(16): 165401

12. J. Tersoff and D. R. Hamann, Theory and application for the scanning tunneling microscope, Phys. Rev. Lett., 1983, 50(25): 1998

13. J. Tersoff and D. R. Hamann, Theory of the scanning tunneling microscope, Phys. Rev. B, 1985, 31(2): 805

14. J. Bardeen, Tunnelling from a many-particle point of view, Phys. Rev. Lett., 1961, 6(2): 57

15. D. Wortmann, S. Heinze, P. Kurz, G. Bihlmayer, and S. Blügel, Resolving complex atomic-scale spin structures by spin-polarized scanning tunneling microscopy, Phys. Rev. Lett., 2001, 86(18): 4132

16. W. A. Hofer and A. Garcia-Lekue, Differential tunneling spectroscopy simulations: Imaging surface states, Phys. Rev. B, 2005, 71(8): 085401

17. T. Kwapiński and M. Jałochowski, Signature of tip electronic states on tunneling spectra, Surf. Sci., 2010, 604(19-20): 1752 
18. H. Ness and F. Gautier, The electronic structure and stability of transition metal nanotips (I), J. Phys.: Condens. Matter, 1995, 7(33): 6625

19. H. Ness and F. Gautier, The electronic structure of transition metal interacting tip and sample and atomic force microscopy (II), J. Phys.: Condens. Matter, 1995, 7(33): 6641

20. H. Ness and F. Gautier, Theoretical study of the interaction between a magnetic nanotip and a magnetic surface, Phys. Rev. B, 1995, 52(10): 7352

21. C. J. Chen, Tunneling matrix elements in three-dimensional space: The derivative rule and the sum rule, Phys. Rev. B, 1990, 42(14): 8841

22. W. Sacks, Tip orbitals and the atomic corrugation of metal surfaces in scanning tunneling microscopy, Phys. Rev. B, 2000, 61(11): 7656

23. C. J. Chen, Effects of $m \neq 0$ tip states in scanning tunneling microscopy: The explanations of corrugation reversal, Phys. Rev. Lett., 1992, 69(11): 1656

24. N. Mingo, L. Jurczyszyn, F. J. Garcia-Vidal, R. Saiz-Pardo, P. L. de Andres, F. Flores, S. Y. Wu, and W. More, Theory of the scanning tunneling microscope: $\mathrm{Xe}$ on $\mathrm{Ni}$ and $\mathrm{Al}$, Phys. Rev. B, 1996, 54(3): 2225

25. F. Calleja, A. Arnau, J. J. Hinarejos, A. L. Vázquez de Parga, W. A. Hofer, P. M. Echenique, and R. Miranda, Contrast reversal and shape changes of atomic adsorbates measured with scanning tunneling microscopy, Phys. Rev. Lett., 2004, 92(20): 206101

26. G. Teobaldi, M. Peñalba, A. Arnau, N. Lorente, and W. A. Hofer, Including the probe tip in theoretical models of inelastic scanning tunneling spectroscopy: $\mathrm{CO}$ on $\mathrm{Cu}(100)$, Phys. Rev. B, 2007, 76(23): 235407

27. A. Garcia-Lekue, D. Sanchez-Portal, A. Arnau, and T. Frederiksen, Simulation of inelastic electron tunneling spectroscopy of single molecules with functionalized tips, Phys. Rev. B, 2011, 83(15): 155417

28. L. Vitali, S. D. Borisova, G. G. Rusina, E. V. Chulkov, and K. Kern, Inelastic electron tunneling spectroscopy: A route to the identification of the tip-apex structure, Phys. Rev. B, 2010, 81(15): 153409

29. C. Sirvent, J. G. Rodrigo, S. Vieira, L. Jurczyszyn, N. Mingo, and F. Flores, Conductance step for a single-atom contact in the scanning tunneling microscope: Noble and transition metals, Phys. Rev. B, 1996, 53(23): 16086

30. M. Büttiker, Y. Imry, R. Landauer, and S. Pinhas, Generalized many-channel conductance formula with application to small rings, Phys. Rev. B, 1985, 31(10): 6207

31. J. Cerdá, M. A. Van Hove, P. Sautet, and M. Salmeron, Efficient method for the simulation of STM images (I): Generalized Green-function formalism, Phys. Rev. B, 1997, 56(24): 15885

32. M. Brandbyge, M. R. Sorensen, and K. W. Jacobsen, Conductance eigenchannels in nanocontacts, Phys. Rev. B, 1997, 56(23): 14956

33. M. Brandbyge, N. Kobayashi, and M. Tsukada, Conduction channels at finite bias in single-atom gold contacts, Phys. Rev. B, 1999, 60(24): 17064

34. A. Bagrets, N. Papanikolaou, and I. Mertig, Ab initio approach to the ballistic transport through single atoms, Phys. Rev. B, 2006, 73(4): 045428

35. A. Bagrets, N. Papanikolaou, and I. Mertig, Conduction eigenchannels of atomic-sized contacts: $\mathrm{Ab}$ initio KKR Green's function formalism, Phys. Rev. B, 2007, 75(23): 235448

36. M. Polok, A. Bagrets, D. V. Fedorov, P. Zahn, and I. Mertig, Evaluation of conduction eigenchannels of an adatom probed by an STM tip, Phys. Rev. B, 2011, 83(24): 245426

37. E. M. L. Plumer, J. van Ek, and D. Weller, The Physics of Ultra-High Density Magnetic Recording, Springer Series in Surface Science Vol. 41, Berlin: Springer, 2001

38. N. Weiss, T. Cren, M. Epple, S. Rusponi, G. Baudot, S. Rohart, A. Tejeda, V. Repain, S. Rousset, P. Ohresser, F. Scheurer, P. Bencok, and H. Brune, Uniform magnetic properties for an ultrahigh-density lattice of noninteracting co nanostructures, Phys. Rev. Lett., 2005, 95(15): 157204

39. D. Serrate, P. Ferriani, Y. Yoshida, S. W. Hla, M. Menzel, K. von Bergmann, S. Heinze, A. Kubetzka, and R. Wiesendanger, Imaging and manipulating the spin direction of individual atoms, Nat. Nanotechnol., 2010, 5(5): 350

40. K. Tao, V. S. Stepanyuk, W. Hergert, I. Rungger, S. Sanvito, and P. Bruno, Switching a single spin on metal surfaces by a STM tip: Ab initio studies, Phys. Rev. Lett., 2009, 103(5): 057202

41. M. Bode, Spin-polarized scanning tunnelling microscopy, Rep. Prog. Phys., 2003, 66(4): 523

42. R. Wiesendanger, Spin mapping at the nanoscale and atomic scale, Rev. Mod. Phys., 2009, 81(4): 1495

43. W. Wulfhekel and C. L. Gao, Investigation of non-collinear spin states with scanning tunneling microscopy, J. Phys.: Condens. Matter, 2010, 22(8): 084021

44. M. Bode, M. Heide, K. von Bergmann, P. Ferriani, S. Heinze, G. Bihlmayer, A. Kubetzka, O. Pietzsch, S. Blügel, and R. Wiesendanger, Chiral magnetic order at surfaces driven by inversion asymmetry, Nature, 2007, 447(7141): 190

45. C. L. Gao, W. Wulfhekel, and J. Kirschner, Revealing the $120^{\circ}$ antiferromagnetic Néel structure in real space: One monolayer Mn on Ag(111), Phys. Rev. Lett., 2008, 101(26): 267205

46. M. Waśniowska, S. Schröder, P. Ferriani, and S. Heinze, Real space observation of spin frustration in $\mathrm{Cr}$ on a triangular lattice, Phys. Rev. B, 2010, 82(1): 012402

47. S. Heinze, K. von Bergmann, M. Menzel, J. Brede, A. Kubetzka, R. Wiesendanger, G. Bihlmayer, and S. Blügel, Spontaneous atomic-scale magnetic skyrmion lattice in two dimensions, Nat. Phys., 2011, 7(9): 713

48. M. Takada, P. L. Gastelois, M. Przybylski, and J. Kirschner, A complex magnetic structure of ultrathin Fe films on Rh (001) surfaces, J. Magn. Magn. Mater., 2013, 329: 95 
49. P. Ferriani, K. von Bergmann, E. Y. Vedmedenko, S. Heinze, M. Bode, M. Heide, G. Bihlmayer, S. Blügel, and R. Wiesendanger, Atomic-scale spin spiral with a unique rotational sense: Mn monolayer on W(001), Phys. Rev. Lett., 2008, 101(2): 027201

50. J. Kudrnovský, F. Máca, I. Turek, and J. Redinger, Substrate-induced antiferromagnetism of a Fe monolayer on the $\operatorname{Ir}(001)$ surface, Phys. Rev. B, 2009, 80(6): 064405

51. A. Deák, L. Szunyogh, and B. Újfalussy, Thicknessdependent magnetic structure of ultrathin $\mathrm{Fe} / \operatorname{Ir}(001)$ films: From spin-spiral states toward ferromagnetic order, Phys. Rev. B, 2011, 84(22): 224413

52. L. Balogh, K. Palotás, L. Udvardi, L. Szunyogh, and U. Nowak, Theoretical study of magnetic domain walls through a cobalt nanocontact, Phys. Rev. B, 2012, 86(2): 024406

53. A. Antal, B. Lazarovits, L. Udvardi, L. Szunyogh, B. Újfalussy, and P. Weinberger, First-principles calculations of spin interactions and the magnetic ground states of $\mathrm{Cr}$ trimers on $\mathrm{Au}(111)$, Phys. Rev. B, 2008, 77(17): 174429

54. L. Udvardi, A. Antal, L. Szunyogh, Á. Buruzs, and P. Weinberger, Magnetic pattern formation on the nanoscale due to relativistic exchange interactions, Physica B, 2008, 403(2-3): 402

55. R. Yang, H. Yang, A. R. Smith, A. Dick, and J. Neugebauer, Energy-dependent contrast in atomic-scale spin-polarized scanning tunneling microscopy of $\mathrm{Mn}_{3} \mathrm{~N}_{2}$ (010): Experiment and first-principles theory, Phys. Rev. B, 2006, 74(11): 115409

56. K. Palotás, W. A. Hofer, and L. Szunyogh, Simulation of spin-polarized scanning tunneling microscopy on complex magnetic surfaces: Case of a $\mathrm{Cr}$ monolayer on $\mathrm{Ag}(111)$, Phys. Rev. B, 2011, 84(17): 174428

57. K. Palotás, Prediction of the bias voltage dependent magnetic contrast in spin-polarized scanning tunneling microscopy, Phys. Rev. B, 2013, 87(2): 024417

58. W. A. Hofer, K. Palotás, S. Rusponi, T. Cren, and H. Brune, Role of hydrogen in giant spin polarization observed on magnetic nanostructures, Phys. Rev. Lett., 2008, 100(2): 026806

59. K. von Bergmann, M. Menzel, D. Serrate, Y. Yoshida, S. Schröder, P. Ferriani, A. Kubetzka, R. Wiesendanger, and S. Heinze, Tunneling anisotropic magnetoresistance on the atomic scale, Phys. Rev. B, 2012, 86(13): 134422

60. N. Néel, S. Schröder, N. Ruppelt, P. Ferriani, J. Kröger, R. Berndt, and S. Heinze, Tunneling anisotropic magnetoresistance at the single-atom limit, Phys. Rev. Lett., 2013, 110(3): 037202

61. N. Néel, J. Kröger, L. Limot, K. Palotás, W. A. Hofer, and R. Berndt, Conductance and Kondo effect in a controlled single-atom contact, Phys. Rev. Lett., 2007, 98(1): 016801

62. K. R. Patton, S. Kettemann, A. Zhuravlev, and A. Lichtenstein, Spin-polarized tunneling microscopy and the Kondo effect, Phys. Rev. B, 2007, 76(10): 100408

63. T. Uchihashi, J. Zhang, J. Kröger, and R. Berndt, Quantum modulation of the Kondo resonance of Co adatoms on
$\mathrm{Cu} / \mathrm{Co} / \mathrm{Cu}(100)$ : Low-temperature scanning tunneling spectroscopy study, Phys. Rev. B, 2008, 78(3): 033402

64. P. Roura-Bas, M. A. Barral, and A. M. Llois, Co impurities on $\mathrm{Ag}$ and $\mathrm{Cu}$ : Kondo temperature dependence on substrate orientation, Phys. Rev. B, 2009, 79(7): 075410

65. K. R. Patton, H. Hafermann, S. Brener, A. I. Lichtenstein, and M. I. Katsnelson, Probing the Kondo screening cloud via tunneling-current conductance fluctuations, Phys. Rev. B, 2009, 80(21): 212403

66. N. Néel, J. Kröger, and R. Berndt, Kondo effect of a Co atom on $\mathrm{Cu}(111)$ in contact with an iron tip, Phys. Rev. B, 2010, 82(23): 233401

67. D. J. Choi, M. V. Rastei, P. Simon, and L. Limot, Conductance-driven change of the Kondo effect in a single cobalt atom, Phys. Rev. Lett., 2012, 108(26): 266803

68. O. Újsághy, J. Kroha, L. Szunyogh, and A. Zawadowski, Theory of the Fano resonance in the stm tunneling density of states due to a single Kondo impurity, Phys. Rev. Lett., 2000, 85(12): 2557

69. L. Gao, W. Ji, Y. B. Hu, Z. H. Cheng, Z. T. Deng, Q. Liu, N. Jiang, X. Lin, W. Guo, S. X. Du, W. A. Hofer, X. C. Xie, and H. J. Gao, Site-specific Kondo effect at ambient temperatures in iron-based molecules, Phys. Rev. Lett., 2007, 99(10): 106402

70. J. A. Aguiar-Hualde, G. Chiappe, E. Louis, and E. V. Anda, Kondo effect in transport through molecules adsorbed on metal surfaces: From Fano dips to Kondo peaks, Phys. Rev. $B, 2007,76(15): 155427$

71. J. A. Aguiar-Hualde, G. Chiappe, E. Louis, E. V. Anda, and J. Simonin, Kondo resonance in the conductance of $\mathrm{CoPc} / \mathrm{Au}(111)$ and $\mathrm{TBrPP}-\mathrm{Co} / \mathrm{Cu}(111)$, Phys. Rev. B, 2009, 79(15): 155415

72. A. E. Miroshnichenko, S. Flach, and Y. S. Kivshar, Fano resonances in nanoscale structures, Rev. Mod. Phys., 2010, 82(3): 2257

73. Y. Yayon, V. W. Brar, L. Senapati, S. C. Erwin, and M. F. Crommie, Observing spin polarization of individual magnetic adatoms, Phys. Rev. Lett., 2007, 99(6): 067202

74. B. W. Heinrich, C. Iacovita, M. V. Rastei, L. Limot, J. P. Bucher, P. A. Ignatiev, V. S. Stepanyuk, and P. Bruno, Spin structure of an atomic protrusion: Probing single atoms on cobalt nanoislands, Phys. Rev. B, 2009, 79(11): 113401

75. L. Zhou, F. Meier, J. Wiebe, and R. Wiesendanger, Inversion of spin polarization above individual magnetic adatoms, Phys. Rev. B, 2010, 82(1): 012409

76. P. Ferriani, C. Lazo, and S. Heinze, Origin of the spin polarization of magnetic scanning tunneling microscopy tips, Phys. Rev. B, 2010, 82(5): 054411

77. J. Wiebe, L. Zhou, and R. Wiesendanger, Atomic magnetism revealed by spin-resolved scanning tunnelling spectroscopy, J. Phys. D Appl. Phys., 2011, 44(46): 464009

78. M. Ternes, A. J. Heinrich, and W. D. Schneider, Spectroscopic manifestations of the Kondo effect on single adatoms, J. Phys.: Condens. Matter, 2009, 21(5): 053001 
79. K. Schouteden, D. A. Muzychenko, and C. Van Haesendonck, Spin-polarized scanning tunneling spectroscopy of self-organized nanoscale Co islands on $\mathrm{Au}(111)$ surfaces, $J$. Nanosci. Nanotechnol., 2008, 8(7): 3616

80. K. Schouteden, E. Lijnen, E. Janssens, A. Ceulemans, L. F. Chibotaru, P. Lievens, and C. Van Haesendonck, Confinement of surface state electrons in self-organized Co islands on $\mathrm{Au}(111)$, New J. Phys., 2008, 10(4): 043016

81. K. Schouteden, D. A. Muzychenko, P. Lievens, and C. Van Haesendonck, Low-temperature scanning tunneling microscopy and spectroscopy investigation of the electronic surface state of self-organized $\mathrm{Cr}$ islands on $\mathrm{Au}(111), \mathrm{J}$. Nanosci. Nanotechnol., 2009, 9(11): 6767

82. K. Schouteden and C. Van Haesendonck, Narrow Au(111) terraces decorated by self-organized Co nanowires: A lowtemperature STM/STS investigation, J. Phys. Condens. Matter, 2010, 22(25): 255504

83. K. Schouteden, K. Lauwaet, D. A. Muzychenko, P. Lievens, and C. Van Haesendonck, Spin-dependent electronic structure of self-organized Co nanomagnets, New J. Phys., 2011, 13(3): 033030

84. A. A. Khajetoorians, J. Wiebe, B. Chilian, and R. Wiesendanger, Realizing all-spin-based logic operations atom by atom, Science, 2011, 332(6033): 1062

85. B. W. Heinrich, C. Iacovita, M. V. Rastei, L. Limot, P. A. Ignatiev, V. S. Stepanyuk, and J. P. Bucher, A spin-selective approach for surface states at Co nanoislands, Eur. Phys. J. $B, 2010,75(1): 49$

86. M. Passoni and C. E. Bottani, Transfer Hamiltonian analytical theory of scanning tunneling spectroscopy, Phys. Rev. $B, 2007,76(11): 115404$

87. F. Donati, S. Piccoli, C. E. Bottani, and M. Passoni, Threedimensional approach to scanning tunneling spectroscopy and application to Shockley states, New J. Phys., 2011, 13(5): 053058

88. G. Rodary, S. Wedekind, H. Oka, D. Sander, and J. Kirschner, Characterization of tips for spin-polarized scanning tunneling microscopy, Appl. Phys. Lett., 2009, 95(15): 152513

89. K. Palotás, W. A. Hofer, and L. Szunyogh, Theoretical study of the role of the tip in enhancing the sensitivity of differential conductance tunneling spectroscopy on magnetic surfaces, Phys. Rev. B, 2011, 83(21): 214410

90. S. Heinze, Simulation of spin-polarized scanning tunneling microscopy images of nanoscale non-collinear magnetic structures, Appl. Phys. A, 2006, 85(4): 407

91. K. Palotás, G. Mándi, and L. Szunyogh, Orbital-dependent electron tunneling within the atom superposition approach: Theory and application to W(110), Phys. Rev. B, 2012, 86(23): 235415

92. W. A. Hofer and A. J. Fisher, Simulation of spin-resolved scanning tunneling microscopy: influence of the magnetization of surface and tip, J. Magn. Magn. Mater., 2003, 267(2): 139
93. A. R. Smith, R. Yang, H. Yang, W. R. L. Lambrecht, A. Dick, and J. Neugebauer, Aspects of spin-polarized scanning tunneling microscopy at the atomic scale: Experiment, theory, and simulation, Surf. Sci., 2004, 561(2-3): 154

94. H. Yang, A. R. Smith, M. Prikhodko, and W. R. L. Lambrecht, Atomic-Scale Spin-Polarized Scanning Tunneling Microscopy Applied to $\mathrm{Mn}_{3} \mathrm{~N}_{2}(010)$, Phys. Rev. Lett., 2002, 89(22): 226101

95. N. D. Lang, Spectroscopy of single atoms in the scanning tunneling microscope, Phys. Rev. B, 1986, 34(8): 5947

96. M. Becker and R. Berndt, Influence of band structure on the apparent barrier height in scanning tunneling microscopy, Phys. Rev. B, 2010, 81(3): 035426

97. M. Becker, and R. Berndt, Contrast inversion of the apparent barrier height of $\mathrm{Pb}$ thin films in scanning tunneling microscopy, Appl. Phys. Lett., 2010, 96(3): 033112

98. W. Tang, E. Sanville, and G. Henkelman, A grid-based Bader analysis algorithm without lattice bias, J. Phys.: Condens. Matter, 2009, 21(8): 084204

99. C. J. Chen, Introduction to Scanning Tunneling Microscopy, Chapter 6, Oxford: Oxford University Press, 1993

100. H. F. Ding, W. Wulfhekel, J. Henk, P. Bruno, and J. Kirschner, Absence of zero-bias anomaly in spin-polarized vacuum tunneling in $\mathrm{Co}(0001)$, Phys. Rev. Lett., 2003, 90(11): 116603

101. A. Tange, C. L. Gao, B. Y. Yavorsky, I. V. Maznichenko, C. Etz, A. Ernst, W. Hergert, I. Mertig, W. Wulfhekel, and J. Kirschner, Electronic structure and spin polarization of the $\mathrm{Fe}(001)-\mathrm{p}(1 \times 1) \mathrm{O}$ surface, Phys. Rev. B, 2010, 81(19): 195410

102. W. Krenner, D. Kühne, F. Klappenberger, and J. V. Barth, Assessment of scanning tunneling spectroscopy modes inspecting electron confinement in surface-confined supramolecular networks, Scientific Reports, 2013, 3: 1454

103. G. Kresse and J. Furthmüller, Efficiency of ab-initio total energy calculations for metals and semiconductors using a plane-wave basis set, Comput. Mater. Sci., 1996, 6(1): 15

104. G. Kresse and J. Furthmüller, Efficient iterative schemes for ab initio total-energy calculations using a plane-wave basis set, Phys. Rev. B, 1996, 54(16): 11169

105. J. Hafner, Ab-initio simulations of materials using VASP: Density-functional theory and beyond, J. Comput. Chem., 2008, 29(13): 2044

106. G. Kresse and D. Joubert, From ultrasoft pseudopotentials to the projector augmented-wave method, Phys. Rev. B, 1999, 59(3): 1758

107. J. P. Perdew and Y. Wang, Accurate and simple analytic representation of the electron-gas correlation energy, Phys. Rev. B, 1992, 45(23): 13244

108. D. Hobbs, G. Kresse, and J. Hafner, Fully unconstrained noncollinear magnetism within the projector augmentedwave method, Phys. Rev. B, 2000, 62(17): 11556

109. D. Hobbs and J. Hafner, Fully unconstrained non-collinear magnetism in triangular $\mathrm{Cr}$ and $\mathrm{Mn}$ monolayers and over- 
layers on $\mathrm{Cu}(111)$ substrates, J. Phys.: Condens. Matter, 2000, 12(31): 7025

110. H. J. Monkhorst and J. D. Pack, Special points for Brillouinzone integrations, Phys. Rev. B, 1976, 13(12): 5188

111. A. Kubetzka, P. Ferriani, M. Bode, S. Heinze, G. Bihlmayer, K. von Bergmann, O. Pietzsch, S. Blügel, and R. Wiesendanger, Revealing antiferromagnetic order of the Fe monolayer on W(001): Spin-polarized scanning tunneling microscopy and first-principles calculations, Phys. Rev. Lett., 2005, 94(8): 087204

112. S. Heinze, S. Blügel, R. Pascal, M. Bode, and R. Wiesendanger, Prediction of bias-voltage-dependent corrugation reversal for STM images of bcc (110) surfaces: W(110), Ta(110), and Fe(110), Phys. Rev. B, 1998, 58(24): 16432

113. S. Heinze, X. Nie, S. Blügel, and M. Weinert, Electric-fieldinduced changes in scanning tunneling microscopy images of metal surfaces, Chem. Phys. Lett., 1999, 315(3-4): 167

114. G. Teobaldi, E. Inami, J. Kanasaki, K. Tanimura, and A. L. Shluger, Role of applied bias and tip electronic structure in the scanning tunneling microscopy imaging of highly oriented pyrolytic graphite, Phys. Rev. B, 2012, 85(8): 085433
115. K. Palotás, and W. A. Hofer, Multiple scattering in a vacuum barrier obtained from real-space wavefunctions, $J$. Phys.: Condens. Matter, 2005, 17(17): 2705

116. K. S. Wang, P. M. Levy, S. F. Zhang, and L. Szunyogh, On the calculation of the magnetoresistance of tunnel junctions with parallel paths of conduction, Philos. Mag. B, 2003, 83(10): 1255

117. M. Ondráček, C. González, and P. Jelínek, Reversal of atomic contrast in scanning probe microscopy on (111) metal surfaces, J. Phys.: Condens. Matter, 2012, 24(8): 084003

118. L. Szunyogh, B. Újfalussy, P. Weinberger, and J. Kollár, Selfconsistent localized KKR scheme for surfaces and interfaces, Phys. Rev. B, 1994, 49(4): 2721

119. R. Zeller, P. H. Dederichs, B. Újfalussy, L. Szunyogh, and P. Weinberger, Theory and convergence properties of the screened Korringa-Kohn-Rostoker method, Phys. Rev. B, 1995, 52(12): 8807

120. G. Rodary, J. C. Girard, L. Largeau, C. David, O. Mauguin, and Z. Z. Wang, Atomic structure of tip apex for spinpolarized scanning tunneling microscopy, Appl. Phys. Lett., 2011, 98(8): 082505 